

\title{
Geophysical Log Data from Basalt Aquifers Near Waipahu on the Island of Oahu and Pahoa on the Island of Hawaii, Hawaii
}

\author{
By Frederick L. Paillet and Alfred E. Hess
}

\section{Abstract}

Two relatively new geophysical logging techniques, the digitally enhanced borehole acoustic televiewer and the heat-pulse flowmeter, were tested from 1987 to 1991 at two sites in Hawaii: Waipahu on the island of Oahu, and Pahoa on the island of Hawaii. Although these data were obtained in an effort to test and improve these two logging techniques, the measurements are of interest to hydrologists studying the aquifers in Hawaii. This report presents a review of the measurements conducted during this effort and summarizes the data obtained in a form designed to make that data available to hydrologists studying the movement of ground water in Hawaiian aquifers. Caliper logs obtained at the Waipahu site indicate the distribution of openings in interbed clinker zones between relatively dense and impermeable basalt flows. The flowmeter data indicate the pattern of flow induced along seven observation boreholes that provide conduits between interbed zones in the vicinity of the Mahoe Pumping Station at the Waipahu site. The televiewer image logs obtained in some of the Waipahu Mahoe boreholes do not show any significant vertical or steeply dipping fractures that might allow communication across the dense interior of basalt flows. Acoustic televiewer logs obtained at the Pahoa site show that a number of steeply dipping fractures and dikes cut across basalt flows. Although flow under ambient hydraulic-head conditions in the Waipahu Mahoe Observation boreholes is attributed to hydraulic gradients associated with pumping from a nearby pumping station, flow in the Waipio Deep Observation borehole on Oahu and flow in the Scientific Observation borehole on Hawaii are attributed to the effects of natural recharge and downward decreasing hydraulic heads associated with that recharge.

\section{INTRODUCTION}

The U.S. Geological Survey has developed various geophysical logging techniques for the characterization of aquifers and has demonstrated the use of these techniques in numerous ground-water investigations. Two recently developed logging techniques are especially useful in studying the movement of ground water through secondary permeability features such as fractures, solution openings, and clinker zones between basalt flows in various bedrock aquifers. These two techniques are the heat-pulse flowmeter (HPFM; Hess, 1986) and the digitally enhanced acoustic borehole televiewer (BHTV; Zemanek and others, 1970, Barton and others, 1991). This report presents the data obtained during 5 years of research logging at two different locations in Hawaii where the HPFM and BHTV logging systems have been used to study flow in fractures and clinker zones in basalt aquifers. The data presented in this report were obtained in conjunction with research efforts conducted by other U.S. Geological Survey scientists (Waipahu, on the island of Oahu), and University of Hawaii scientists (Pahoa, on the island of Hawaii). The primary focus of the research involving the HPFM and BHTV in these efforts was the development and improvement of the two logging techniques for studies in the hydrogeology of Hawaiian basalts. The technical improvement of the 
HPFM system and advances in BHTV log interpretation have been reported in a series of reports and publications (Hess, 1986; Hess and Paillet, 1990b; Paillet and others, 1985, 1990; Paillet, 1991). However, none of the specific results from the Waipahu and Pahoa field sites have been reported in the literature as of the date of this report (1995). This report summarizes the data obtained during 5 years of logging at the Waipahu and Pahoa research sites (1987-1991). The report provides geophysical data in a form that is useful to hydrogeologists interested in the movement of ground water through basalt flows at sites similar to the two study sites (Visher and Mink, 1964). The data are presented with enough interpretation to make the results of the BHTV and HPFM logging understandable to the hydrogeologist not familiar with the operation of this equipment or the interpretation of BHTV and HPFM log data. This mode of presentation is designed to provide a complete and self-sufficient reference on these data, so that the hydrogeologist interested in the hydraulic properties of the aquifers at the two study sites will be able to make use of this information without reference to any additional publications in the geophysical literature.

Clifford Voss of the U.S. Geological Survey provided the temperature and single-point resistivity logs for the Waipahu boreholes and assisted in the field work at the Waipahu study site. The Hawaii Natural Energy Institute, University of Hawaii, provided access to the Scientific Observation borehole SO-2 at Pahoa and made data derived from drilling and coring available during the course of the logging project. The assistance of Dr. Harry Olson of the Hawaii Natural Energy Institute, and Dr. Don Thomas of the University of Hawaii in gaining access to the Pahoa site and in various other activities related to logging operations is greatly appreciated. Dr. Colleen Barton, Department of Geophysics, Stanford University, California, provided the digitization of televiewer data from both the Waipahu and Pahoa sites, and her efforts contributed to the data displayed in figures 13 and 25.

\section{DESCRIPTION OF GEOPHYSICAL LOGS}

\section{Conventional Geophysical Logs}

Conventional geophysical well logs provide continuous profiles of the properties of aquifers by recording measurements made as a geophysical probe is moved along the well bore. Caliper, temperature, single-point resistance, natural gamma, and spinner flowmeter logs were made during the study described in this report at either the Waipahu or Pahoa sites. These types of logs are described in detail in standard geophysical logging texts by Keys (1990) and Hearst and Nelson (1985). The following discussion is limited to topics in conventional log analysis that are directly related to the interpretation of the logs obtained at the Waipahu and Pahoa sites.

Caliper logs record the average extension of three coupled spring-loaded arms as the logging tool is moved along the well bore. The caliper log gives a profile of the average borehole diameter, indicating depths where the borehole is enlarged during drilling, or where naturally occurring openings intersect the borehole wall. However, the caliper arms are often too large to fit into individual fracture openings unless the drilling process enlarges the borehole where the fracture plane intersects the well bore. For this reason, local borehole wall enlargements almost always provide an indirect indication of fractures through the effect of fracturing on the strength of rocks adjacent to the borehole wall rather than a direct indication of fracture openings (Keys, 1979).

Temperature and single-point resistance logs measure the temperature of the fluid filling the borehole and the resistance of the borehole fluid and surrounding formation, respectively. These properties may sometimes be related to the temperature of the surrounding rock, or to the electrical resistivity of the water saturating the adjacent aquifer (Keys, 1990). If water is moving along the borehole between separate openings intersecting the well bore, the temperature and electrical resistivity measured at a given depth will be determined by the properties of the water saturating the aquifer where the water entered the well bore, and not the properties of the water saturating the aquifer immediately adjacent to the measurement point. For this reason, temperature and single-point resistance logs can be very useful in identifying the points where water is entering or exiting the borehole under ambient conditions or during aquifer tests. These entry and exit points are generally associated with changes in slope in the temperature and single-point resistance logs. The single-point resistance probe used in this study is a specially designed probe developed by Clifford Voss of the U.S. Geological Survey for use in Hawaiian boreholes. The logs 
obtained with that probe can be calibrated in borehole fluid resistivity, but that analysis is beyond the scope of this report. The single-point resistance logs presented in this report are provided as qualitative indicators of borehole fluid resistivity in ohms rather than as quantitative logs of borehole fluid resistivity in ohm-meters. All such logs are labelled "single-point resistivity logs" in this report, and are treated in a manner analagous to the conventional and differential single-point logs described by Keys (1990).

The natural-gamma log records the gamma activity of geological formations and is used to identify variations in lithology along the well bore. One of the most important considerations in gamma logging is the time interval used to average gamma counts in order to compute an average gamma activity measurement. In general, the averaging time needs to be large enough to provide a repeatable average so that the natural statistical properties of the gamma emission do not appear larger than the variations in average gamma emission rate associated with different geological formations.

The spinner flowmeter log uses an impeller aligned transverse to the borehole to measure the rate of water movement along the well bore. The rate at which the impeller turns (in rotations per second) is linearly related to the velocity of water movement along the well bore. The impeller is run centralized in the borehole to insure that the same part of the parabolic flow profile is measured at each point on the log. The most important limitation of conventional flowmeter logging is the relatively large flow rate required to turn the impeller. In general, impeller flowmeters need a flow corresponding to an average borehole fluid velocity of about 5 feet per minute or greater to give useful measurements. Slightly better flow resolution can sometimes be obtained by recording trolling flow measurements as the flowmeter is moved up and down the borehole, and subtracting the two sets of measurements to estimate the net flow along the borehole.

\section{Acoustic Borehole Televiewer (BHTV) Logs}

The BHTV log produces a photographic image of the borehole wall that indicates the intensity of acoustic reflections from an acoustic source scanning the borehole wall at about 3 rotations per second (Zemanek and others, 1970; Paillet and others, 1990). The source transducer uses a $1.25 \mathrm{MHz}$ signal and has a beam width of about 0.3 in. At those depths where fractures intersect the borehole wall, the intersection scatters acoustic energy, which produces a linear feature on the photographic output from the BHTV. The orientation of these features can be used to infer strike and dip of fractures with respect to the borehole axis (fig. 1). The azimuthal orientation of the televiewer image is determined by the local direction of magnetic north. Laboratory calibrations and other equipment checks indicate that the BHTV horizontal sweeps are triggered within a few degrees of the component of the Earth's magnetic field in the plane transverse to the borehole axis. If required, true north on the BHTV logs may be determined by applying corrections for local geomagnetic declination and borehole inclination (Kierstein, 1983). 

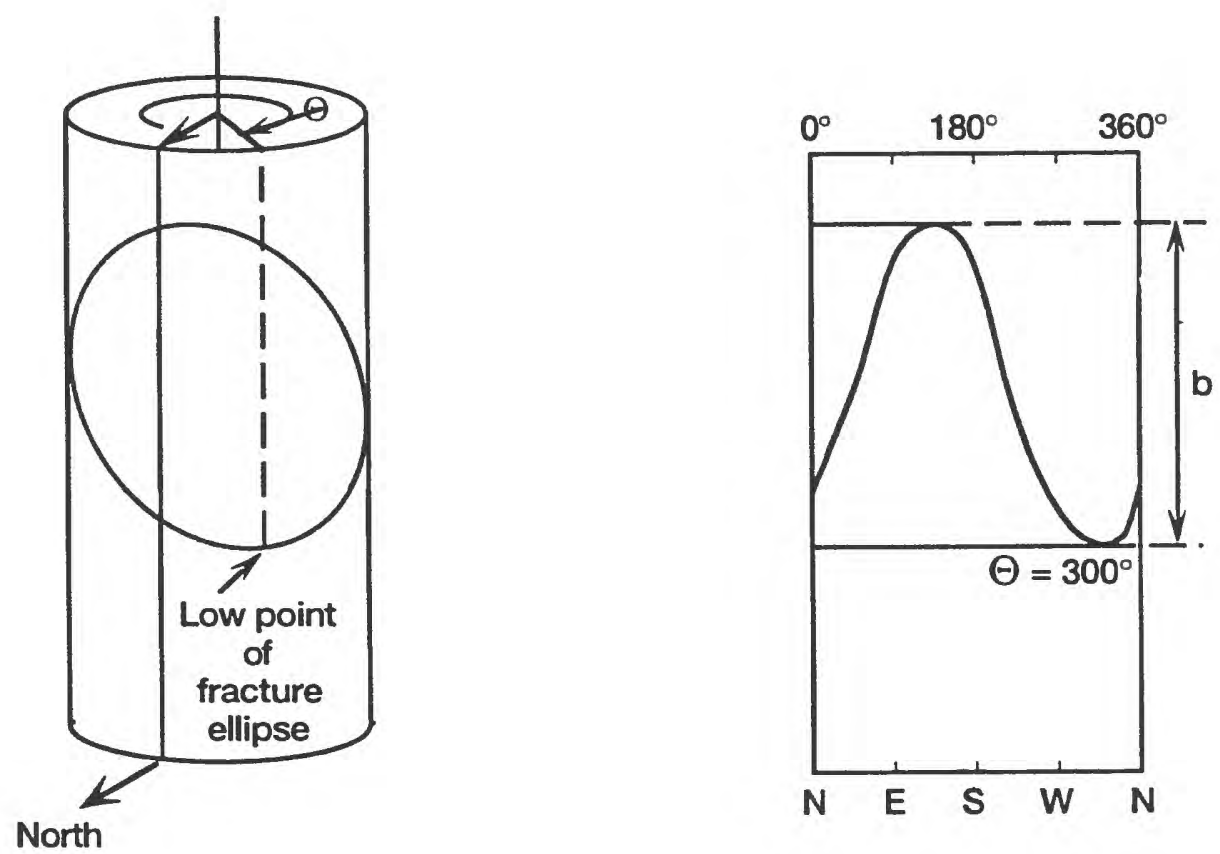

$$
\begin{aligned}
& \text { Dip }=\tan ^{-1} \frac{b}{\text { diameter }} \\
& \text { Strike }=\Theta \pm 90^{\circ}
\end{aligned}
$$

Figure 1. A fracture intersecting a cylindrical borehole, and the interpretation of fracture strike and dip from the acoustic borehole televiewer image log. 
BHTV logs provide a qualitative indication of fracture size because fractures with larger apertures appear as wider, darker features on the BHTV display. The width of the linear feature associated with a fracture can be inferred from the scale of the photographic output of the logging system, but the width of these lines represents fracture aperture at the borehole wall convolved with the 0.3 -in.-wide source beam. It also is probable that the fracture width at the borehole wall has been enlarged during drilling, so an aperture measured there would not be representative of the fracture aperture in the undamaged formation. Specific examples of the interpretation of fracture aperture using BHTV logs are given by Keys (1979), Paillet and others (1985), and Paillet (1991).

\section{Heat-Pulse Flowmeter (HPFM) Logs}

The HPFM was developed to provide a borehole flowmeter with greater sensitivity than conventional impeller flowmeters. The HPFM measures axial flows along the borehole at discrete depths by detecting the time required for a small volume of thermally tagged water to travel from the heat grid to a thermistor in the measurement section of the tool (Hess, 1986) (fig. 2). The sensitivity of the HPFM is increased by the use of a downhole system that uses an inflatable packer to block the annulus around the tool, which forces all flow through the measurement section (Hess and Paillet, 1990a). With the packer inflated, the inverse of heat-pulse travel time is calibrated in terms of borehole discharge in gallons per minute (fig. 3). Several slightly different calibrations were used over the period of this study because tool configurations and components changed as the equipment was modified over time to improve HPFM performance. Experiments at the U.S. Geological Survey flowmeter calibration facility demonstrate that the HPFM can detect flows as slow as $0.01 \mathrm{gal} / \mathrm{min}$. (Hess and Paillet, 1990b). 


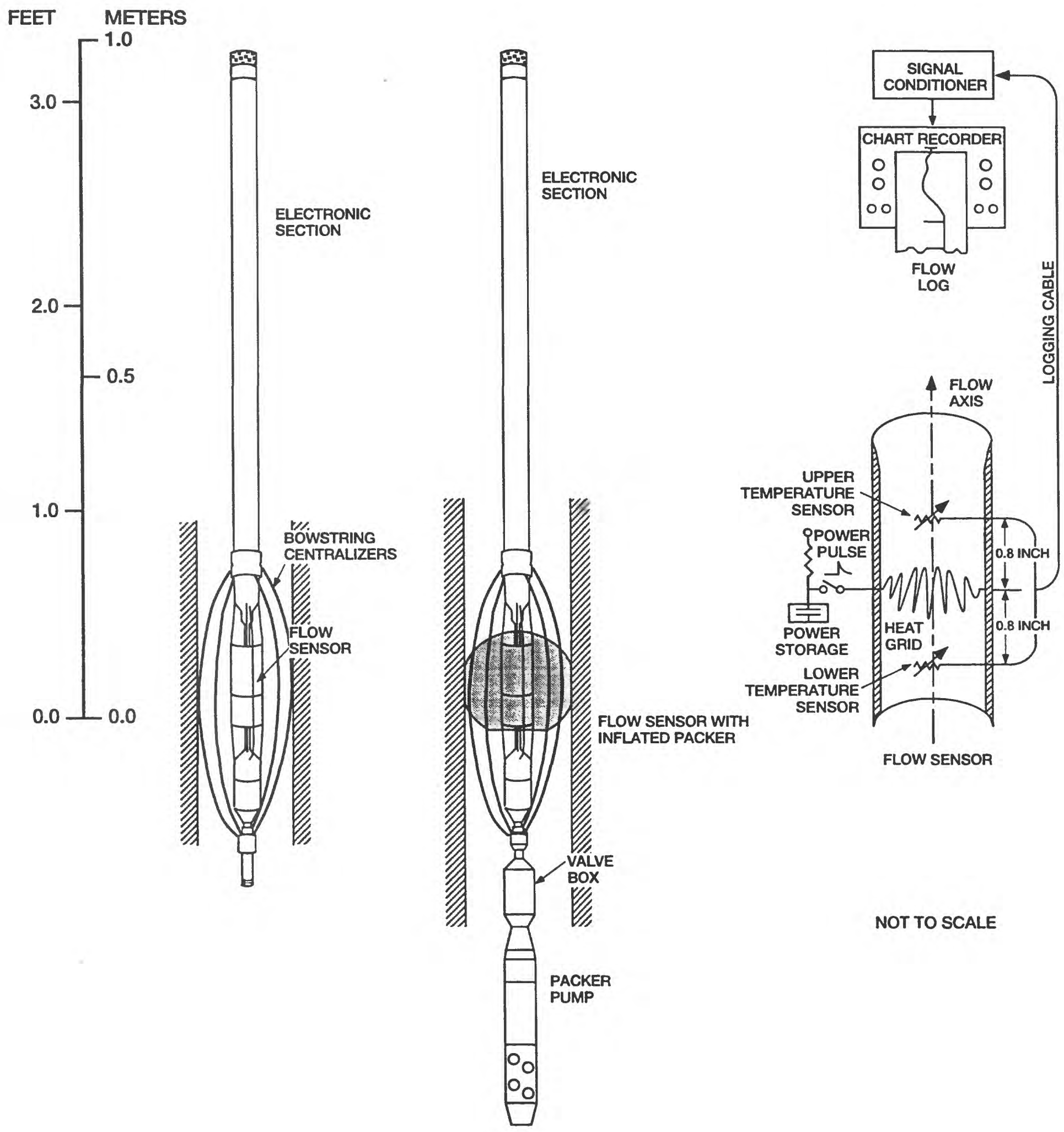

Figure 2. Heat-pulse flowmeter. 


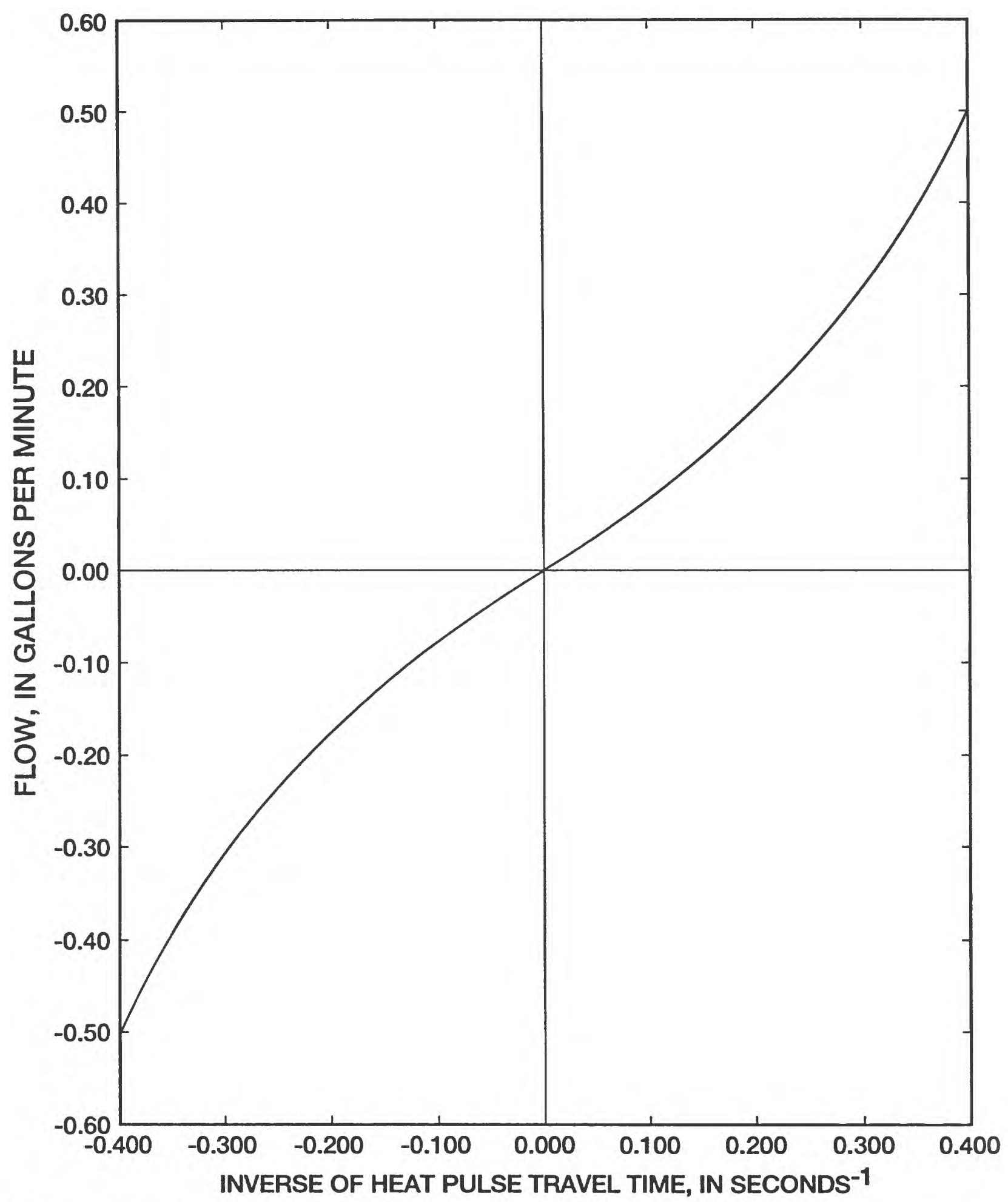

Figure 3. Typical calibration chart for a heat-pulse flowmeter with an inflated packer in place in a 6-inch diameter column. With an inflated packer, the flowmeter calibration is largely independent of the diameter of a borehole; without the packer, the flowmeter calibration is strongly dependent on the borehole diameter. 
The HPFM can be operated in two modes: (1) a fixed-depth mode with or without a flow concentration packer and (2) a trolling mode without a packer or with the packer uninflated in place around the measurement section. The first mode with the packer inflated provides maximum accuracy and resolution of flows, but the measurement process is slowed by the time required to inflate and deflate the packer at each measurement station. The second, the trolling mode without the packer inflated, produces measurements that are less accurate than the fixeddepth measurements with packer inflated, but can be made while trolling the logging tool in the well bore. The trolling measurements are inherently less accurate than those made with the packer inflated, and the trolling measurements are offset by a constant bias imposed by the fixed trolling speed and borehole diameter. In either mode of operation, the HPFM measures only the vertical component of flow in the borehole, whereas a significant horizontal component of flow would occur where boreholes are intersected by permeable beds and fracture zones.

The Waipahu measurements were made using both the fixed-depth and trolling modes of HPFM logging during the course of the field experiments described in this report. The fixed-depth HPFM measurements gave accurate measurements of flow along the borehole at fixed depths where the packer was inflated to force all flow through the measurements section of the logging tool. The log of measurements obtained while trolling the logging tool was used to identify depths where there were abrupt changes in the measured flows, indicating inflow or outflow from the borehole. The two modes of measurements can be effectively combined by using a series of fixed-depth measurements to obtain accurate absolute values for the flow at discreet depths. The profile of relative flow given by the trolling mode measurements can then be shifted to match the limited number of fixed-depth measurements. This procedure effectively compensates for the measurement bias induced by the trolling speed in the trolling mode. 


\section{GEOPHYSICAL LOGGING NEAR WAIPAHU, OAHU}

\section{Overview of Logging Activities}

The Waipahu Mahoe Observation boreholes were located in the vicinity of a municipal well field (the Mahoe Pumping Station constructed in 1964) that was pumping water at rates up to several millions of gallons per day (fig. 4) during the logging described in this report. Several other wells also known to be intermittently pumped were within a mile of the site, including one large capacity well operated by a sugar mill in the town of Waipahu. HPFM logs were run at the Waipahu Mahoe site in boreholes Obs A, Obs B, Obs C, Obs D, Obs E, Obs F, and Obs $\mathrm{H}$ at various times over the period 1987-90, and these data were compared to fluid temperature, fluid conductivity, and caliper logs run in the same boreholes over these periods. These boreholes were nominally 7-3/8 inches in diameter and ranged from 300 to 450 feet in depth. Relatively short steel surface casings were used to stabilize the upper parts of the boreholes, so that water level in all of the boreholes occurred in the open borehole below the bottom of casing. All of the Waipahu Mahoe Observation boreholes subsequently have been cemented and abandoned. The location of each of the Waipahu Mahoe Observation boreholes with respect to the four production wells at the Mahoe Pumping Station are listed in table 1. 


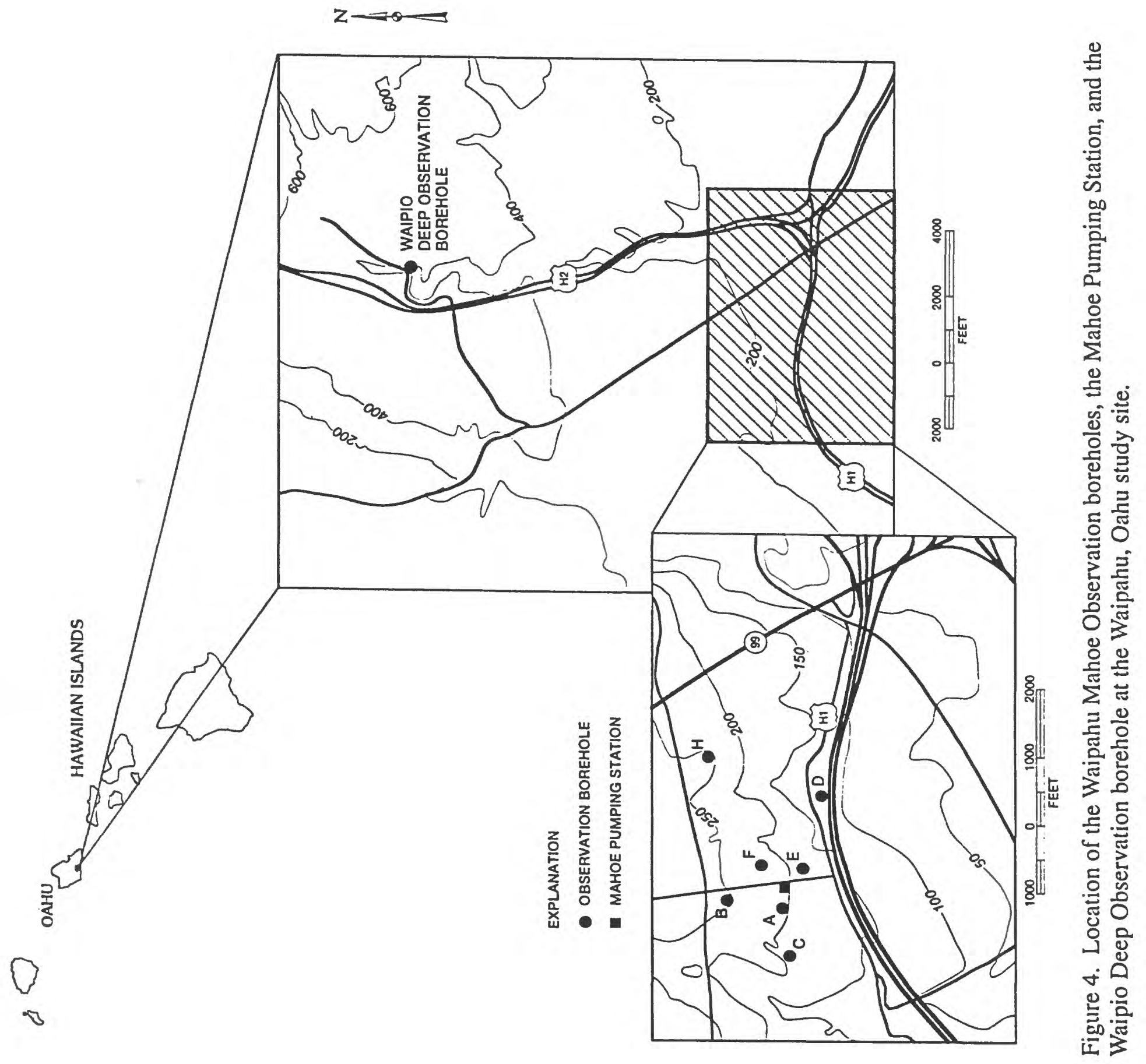




\section{Geophysical Log Data from Basalt Aquifers}

near Waipahu on the Island of Oahu and Pahoa

on the Island of Hawaii, Hawaii

By Frederick L. Paillet ${ }^{1}$ and Alfred E. Hess ${ }^{2}$

U.S. GEOLOGICAL SURVEY

Open-File Report 95-383

${ }^{1}$ U.S. Geological Survey, Denver, Colorado

${ }^{2}$ Boulder, Colorado

Denver, Colorado

1995 
Logs were also obtained in the Waipio Deep Observation borehole located a little more than a mile northeast of the other Waipahu Mahoe Observation boreholes (fig. 4). This borehole is 8 inches in diameter, is more than 1600 feet deep, and extends below the freshwater/salt-water interface. HPFM logs were obtained in the Waipio Deep Observation borehole in 1989. Because of time constraints on field work, most HPFM data were obtained by logging in the trolling mode. Measurements made below the freshwater/salt-water interface in this borehole were the first ever made in saline water with the HPFM. Initial attempts to measure flow in this borehole were unsuccessful because electrically conductive saline water affected the electrical connections in the flowmeter. After minor redesign of the HPFM, flow measurements were made at all depths in the Waipio Deep Observation borehole, demonstrating the capability of the HPFM to operate in saline water. 
Table 1. Locations and separations of Mahoe Pumping Station production wells and Waipahu Mahoe Observation boreholes

\begin{tabular}{|c|c|c|c|c|c|c|c|c|c|c|c|}
\hline \multirow{3}{*}{ Borehole } & \multicolumn{2}{|c|}{ Coordinates $^{\top}$} & \multirow{3}{*}{$\begin{array}{c}\text { Surface }^{2} \\
\text { Elevations }\end{array}$} & \multicolumn{8}{|c|}{ Separations and Headings } \\
\hline & \multirow{2}{*}{$\begin{array}{l}\text { North- } \\
\text { South }\end{array}$} & \multirow{2}{*}{$\begin{array}{l}\text { East- } \\
\text { West }\end{array}$} & & \multicolumn{2}{|c|}{ Prod1 } & \multicolumn{2}{|c|}{ Prod2 } & \multicolumn{2}{|c|}{ Prod3 } & \multicolumn{2}{|c|}{ Prod4 } \\
\hline & & & & Dist $^{3}$ & Angle 4 & Dist $^{3}$ & Angle 4 & Dist ${ }^{3}$ & Angle 4 & Dist $^{3}$ & Angle \\
\hline \multicolumn{12}{|c|}{ Production Wells } \\
\hline Prod1 & $45 S$ & $97 \mathrm{~W}$ & 201.5 & Ref & Ref & 60 & 82.4 & 167 & 125.6 & 214 & 114.5 \\
\hline Prod2 & $52 S$ & $37 \mathrm{~W}$ & 201.5 & 60 & -97.6 & $\operatorname{Ref}$ & Ref & 130 & 144.1 & 166 & 125.6 \\
\hline Prod3 & $53 \mathrm{~N}$ & $38 \mathrm{E}$ & 202.3 & 167 & -54.4 & 129 & -35.8 & Ref & Ref & 60 & 81.8 \\
\hline Prod4 & $44 \mathrm{~N}$ & $98 \mathrm{E}$ & 202.4 & 214 & -65.5 & 166 & -54.4 & 60 & -98.2 & Ref & Ref \\
\hline \multicolumn{12}{|c|}{ Observation Boreholes } \\
\hline Obs A & $32 \mathrm{~N}$ & $182 \mathrm{E}$ & 204.2 & 290 & -74.7 & 236 & -68.9 & 145 & -98.2 & 86 & -98.1 \\
\hline Obs B & $1005 \mathrm{~N}$ & $144 \mathrm{E}$ & 240.5 & 1077 & -13.0 & 1073 & -9.6 & 958 & -6.3 & 962 & -2.8 \\
\hline Obs C & $37 \mathrm{~N}$ & $578 \mathrm{E}$ & 194.1 & 680 & -83.1 & 622 & -81.7 & 540 & -91.7 & 481 & -90.8 \\
\hline Obs D & $361 S$ & $1417 \mathrm{E}$ & 129.6 & 1357 & 103.5 & 1414 & 102.6 & 1513 & 105.9 & 1568 & 105.0 \\
\hline Obs E & $270 S$ & $240 \mathrm{E}$ & 191.0 & 267 & 147.6 & 298 & 137.0 & 427 & 139.1 & 462 & 132.9 \\
\hline Obs F & $321 \mathrm{~N}$ & $234 \mathrm{E}$ & 216.7 & 390 & 20.6 & 422 & 27.8 & 382 & 45.5 & 432 & 50.2 \\
\hline Obs H & $1315 \mathrm{~N}$ & $1647 \mathrm{E}$ & 240.0 & 2062 & 48.8 & 2113 & 49.7 & 2106 & 53.1 & 2159 & 54.0 \\
\hline Waipio & $12063 \mathrm{~N}$ & $4135 W$ & 400.0 & 12763 & 18.4 & 12789 & 18.7 & 12715 & 19.1 & 12742 & 19.4 \\
\hline
\end{tabular}


BHTV logs were run in four of the Waipahu Mahoe boreholes (Obs A, Obs B, Obs C, and Obs D) in April 1990 in an effort to learn about the structure of openings identified on the caliper logs and to determine whether the nearly horizontal basalt flows encountered by the boreholes contained steeply dipping fractures that could allow hydraulic communication between interbed zones. The BHTV logs were obtained by modifying cablehead connections on the televiewer probe used on the mainland to fit a four-conductor logging cable and winch available in Hawaii. A poorly understood incompatibility between the BHTV system and the winch and generator on the logging truck could not be resolved during the period available for logging and caused a rhythmic noise signal to be superimposed on the BHTV log data. This noise degraded the quality of BHTV data, but some information could still be obtained from the logs.

\section{Conventional Logs for the Waipahu Mahoe Boreholes}

Conventional temperature, single-point resistance, and caliper logs for the Waipahu boreholes are given in figures 5-11. These logs were obtained by Clifford Voss of the U.S. Geological Survey using specially designed conventional caliper and temperature probes and a specially designed single-point resistance probe and are provided for comparison with HPFM and BHTV data. These logs indicate that ground water was flowing through the boreholes between certain of the openings identified on the caliper logs at the time of logging. The points where water was entering or exiting the boreholes are indicated by abrupt changes in slope of the temperature and single-point resistance logs. However, these interpretations are difficult to make because there can be a great deal of ambiguity or nonuniqueness in the interpretation of these logs (Keys, 1990). 


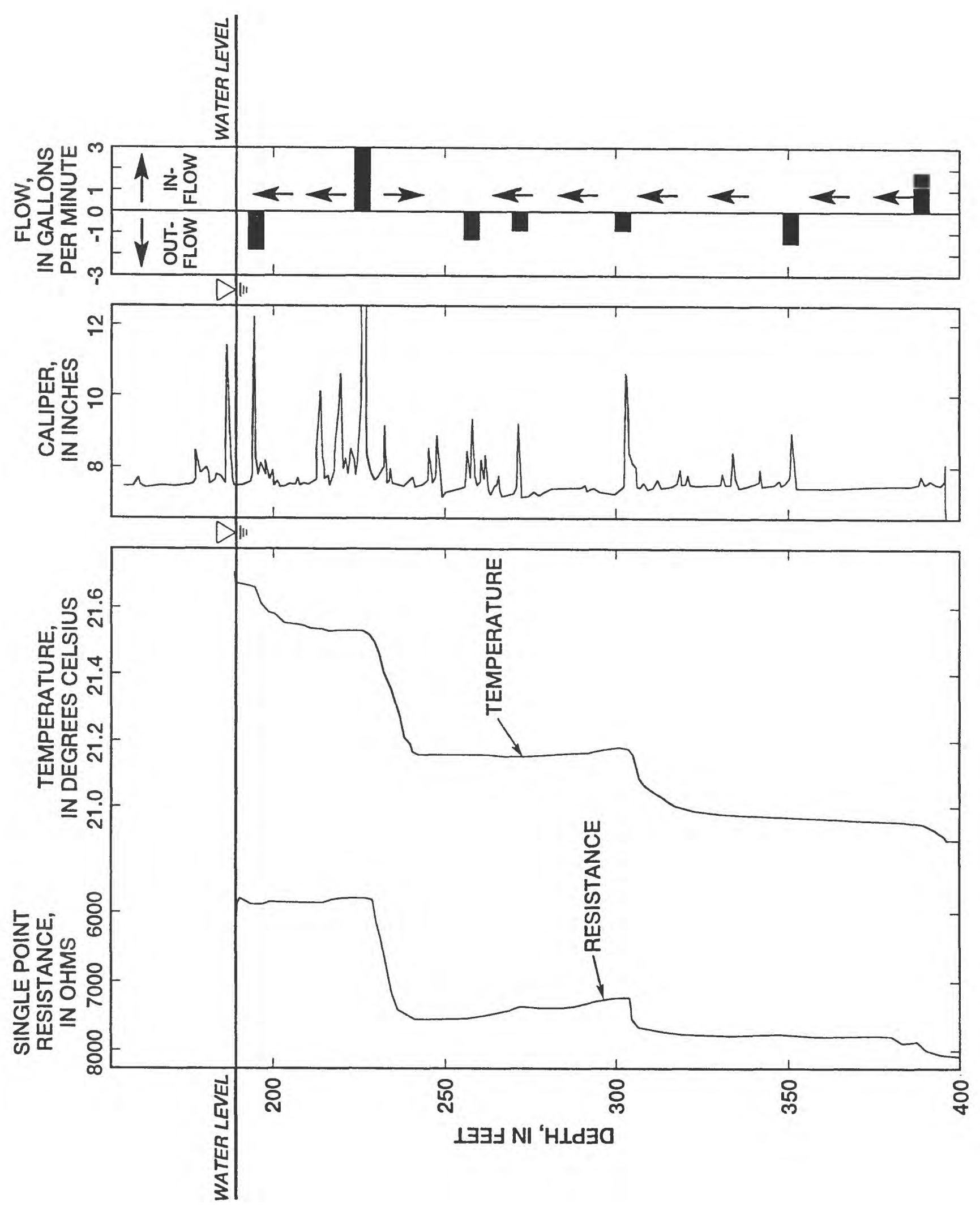

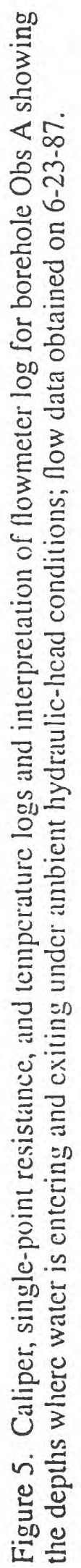




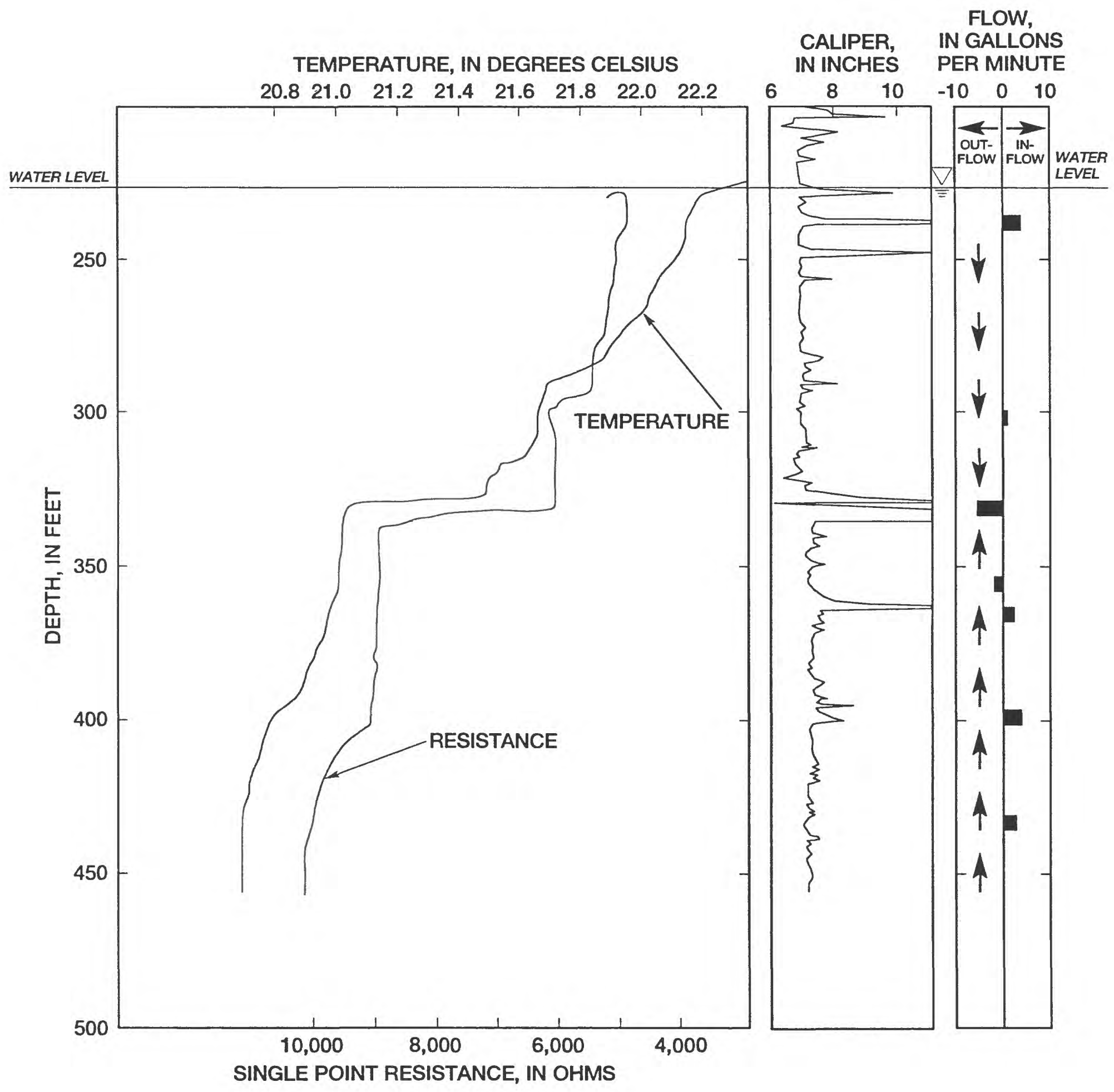

Figure 6. Caliper, single-point resistance, and temperature logs and interpretation of flowmeter log for borehole Obs B showing the depths where water is entering and exiting under ambient hydraulic-head conditions; flow data obtained on 6-30-87. 


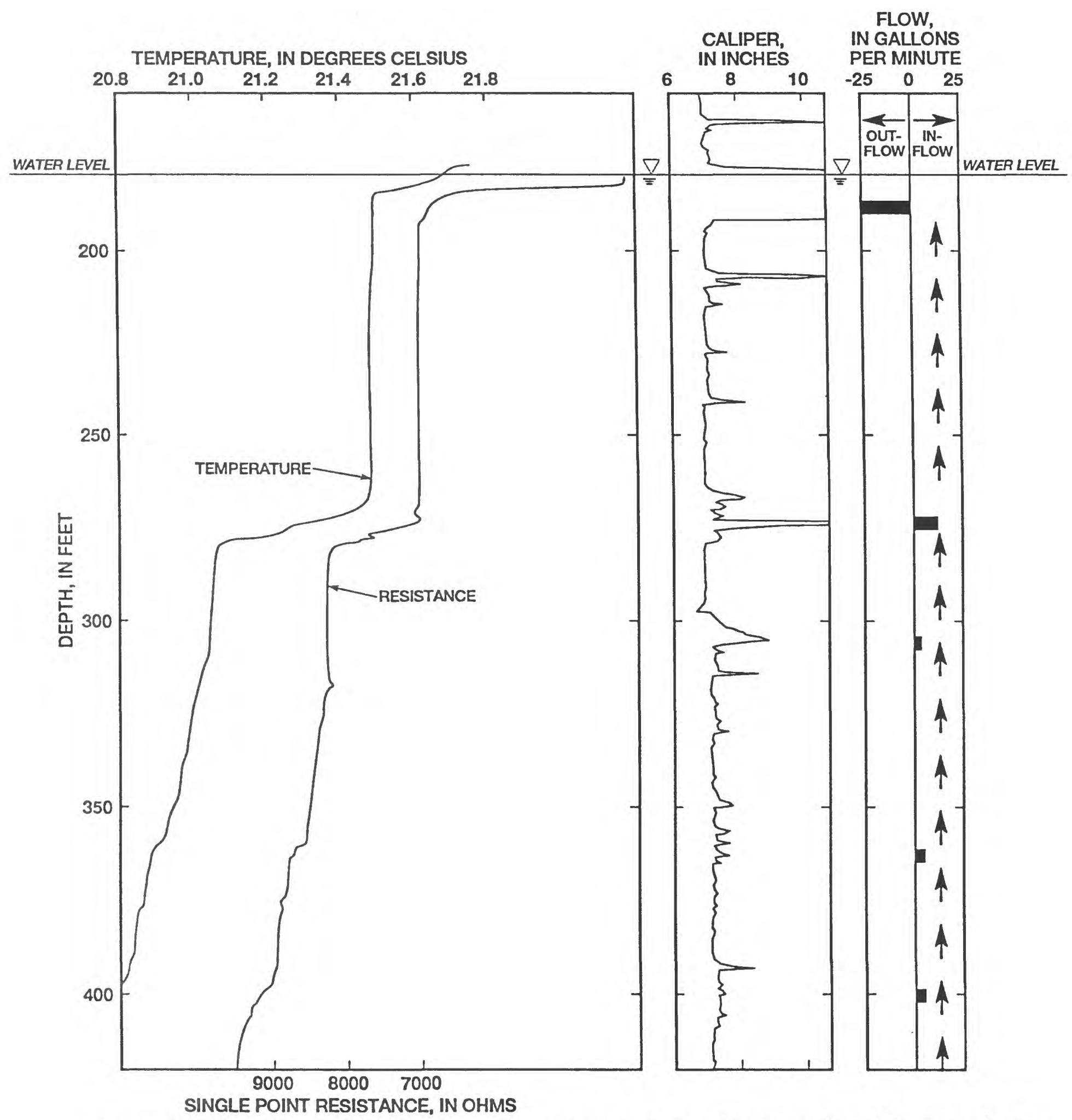

Figure 7. Caliper, single-point resistance, and temperature logs and interpretation of flowmeter log for borehole $\mathrm{Obs} \mathrm{C}$ showing the depths where water is entering and exiting under ambient hydraulic-head conditions; flow data obtained on 7-22-88. 

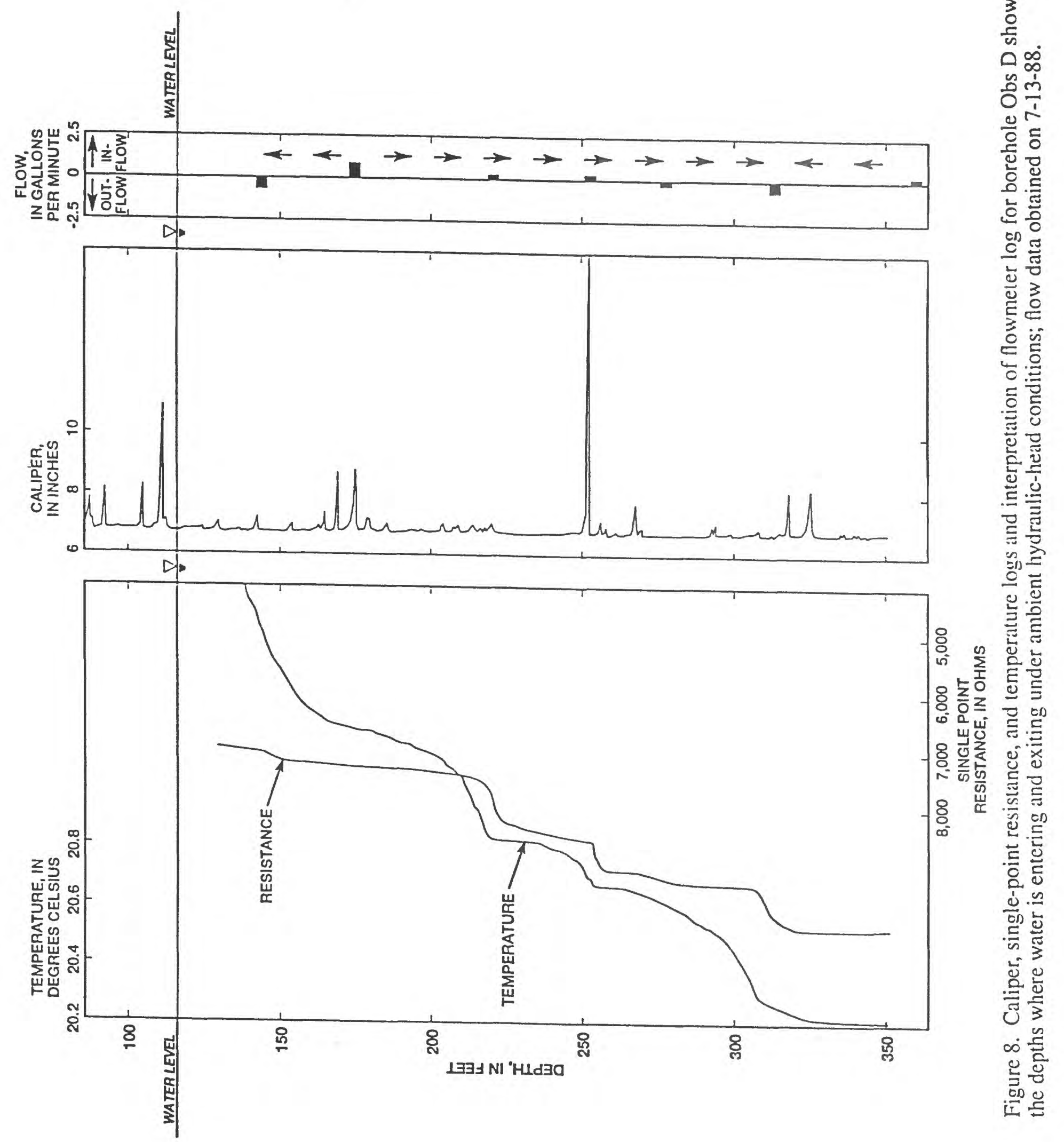
FLOW,

TEMPERATURE, IN

DEGREES CELSIUS

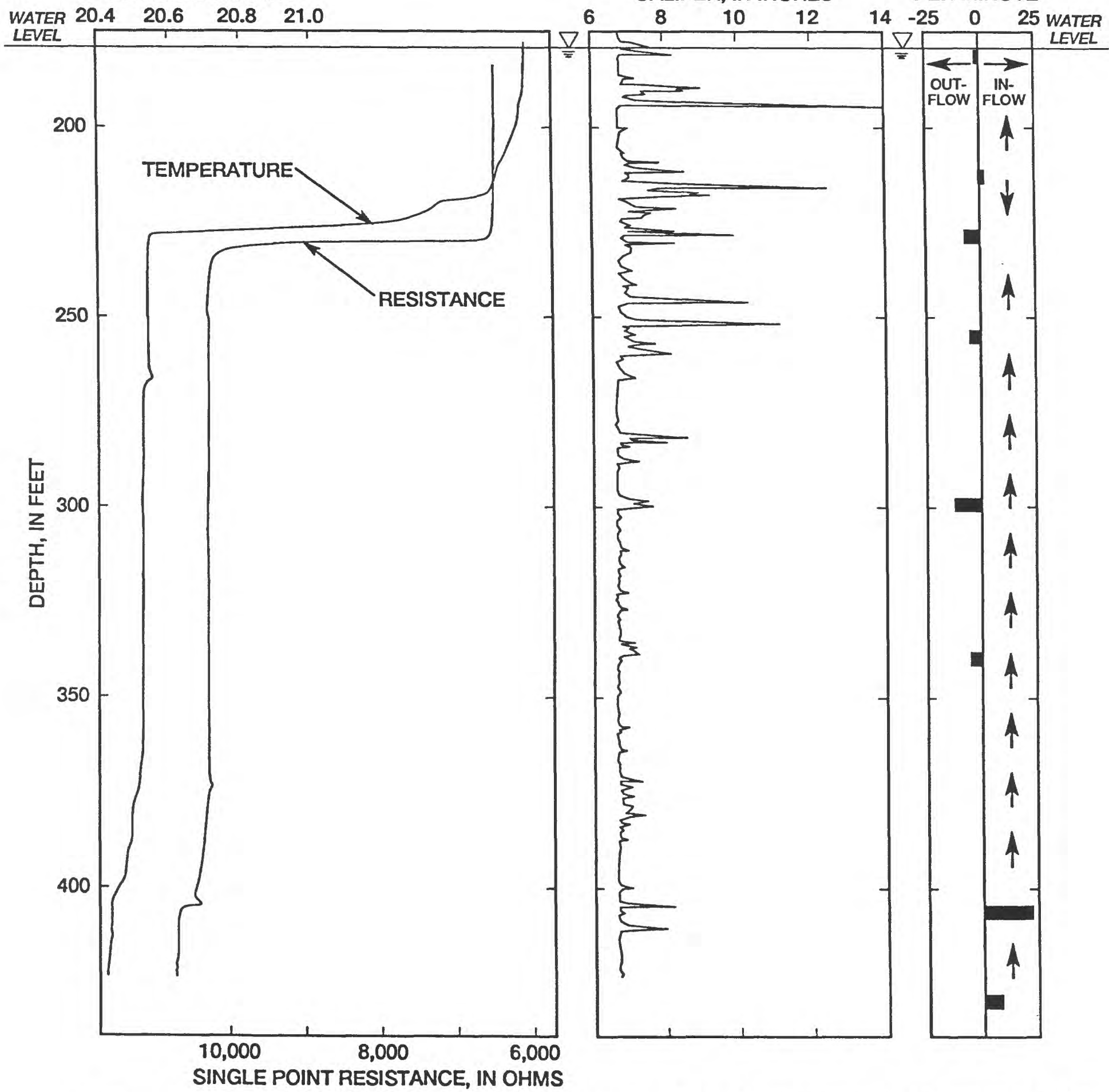

Figure 9. Caliper, single-point resistance, and temperature logs and interpretation of flowmeter log for borehole Obs E showing the depths where water is entering and exiting under ambient hydraulic-hend conditions; llow data oblained on 7-20-88. 


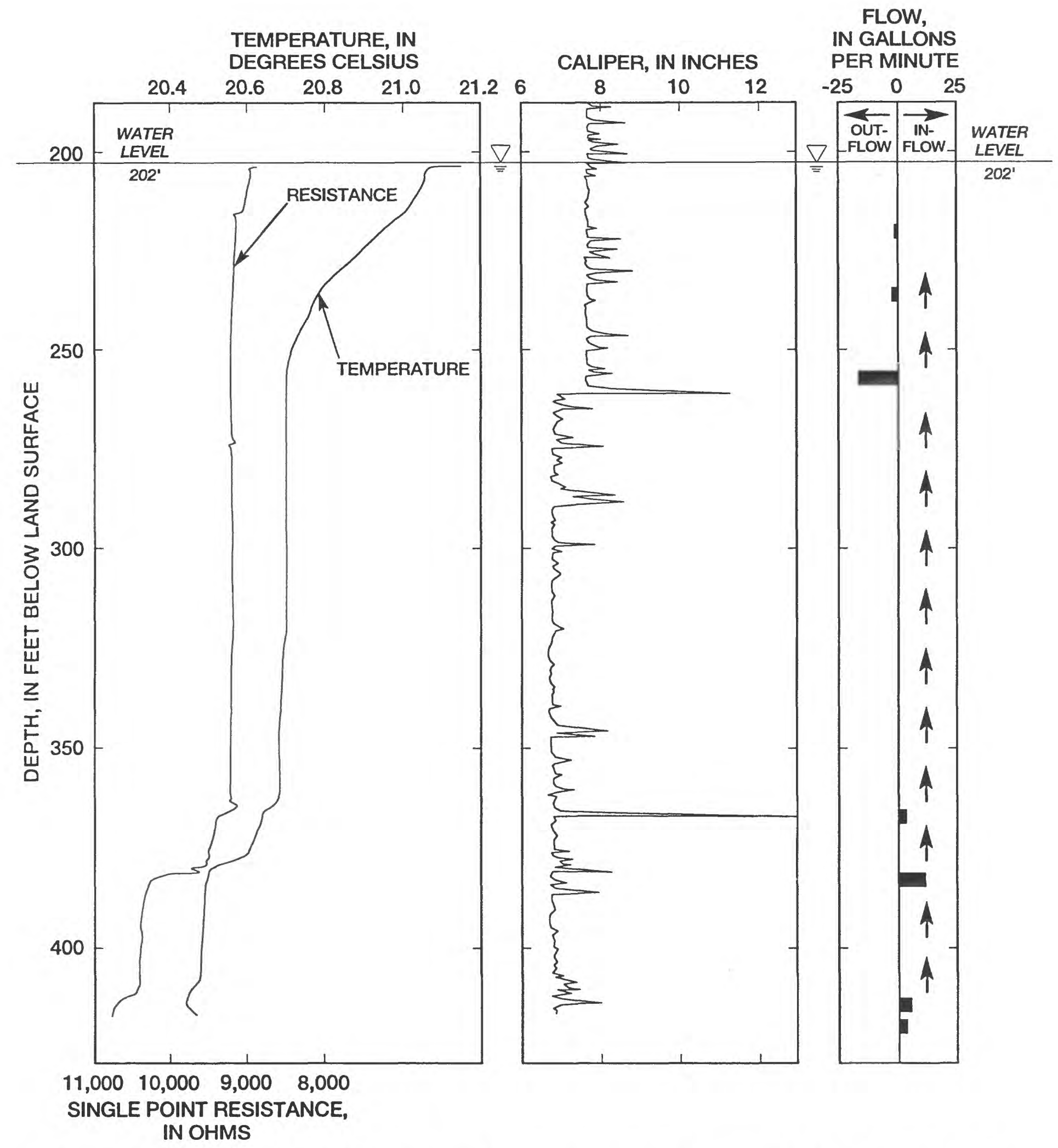

Figure 10. Caliper, single-point resistance, and temperature logs and interpretation of flowmeter log for borehole $\mathrm{Obs} F$ showing the depths where water is entering and exiting under ambient hydraulic-head conditions; flow data obtained on 7-15-88. 


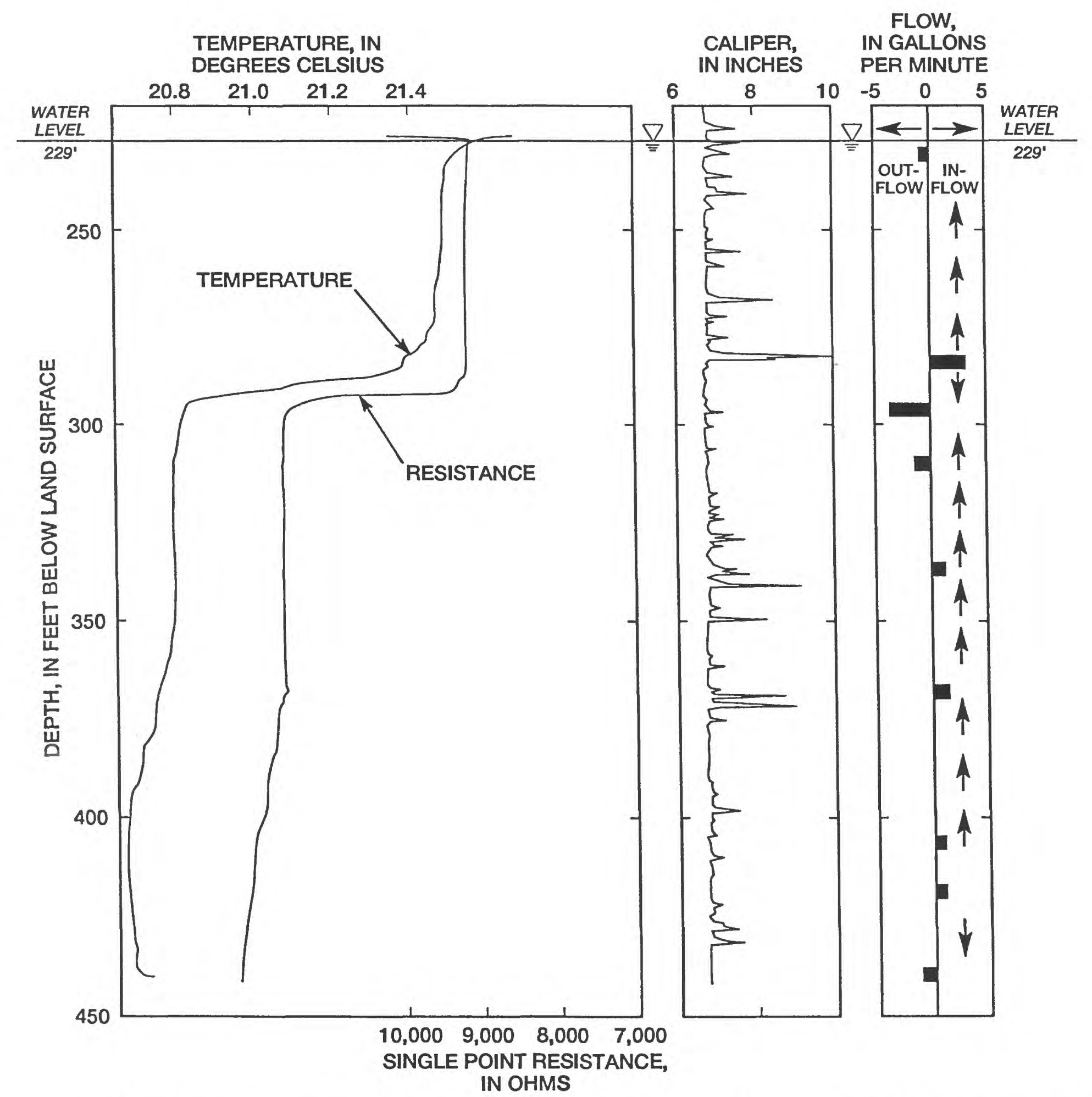

Figure 11. Caliper, single-point resistance, and temperature logs and interpretation of flowmeter log for borehole $\mathrm{Obs} \mathrm{H}$ showing the depths where water is entering and exiting under ambient hydraulic-head conditions; flow data obtained on 7-26-88. 


\section{U.S. DEPARTMENT OF THE INTERIOR BRUCE BABBITT, Secretary}

U.S. GEOLOGICAL SURVEY

Gordon P. Eaton, Director

The use of trade, product, industry, or firm names is for descriptive purposes only and does not imply endorsement by the U.S. Government.

For additional information write to:

Copies of this report can be purchased from:

Chief, Branch of Regional Research

U.S. Geological Survey

U.S. Geological Survey

Earth Science Information Center

Box 25046, MS 418

Open-File Reports Section

Denver Federal Center

Box 25286, MS 517

Denver, CO 80225

Denver Federal Center

Denver, CO 80225 


\section{Acoustic Borehole Televiewer Logs for the Waipahu Mahoe Boreholes}

BHTV logs obtained in two of the Waipahu Mahoe boreholes are compared to the caliper log in figure 12. Representative intervals of BHTV log were recorded on a conventional videorecorder, so that the signal could be digitized and subjected to various image enhancement techniques. An example of such data is shown in figure 13. The data show borehole reflections given separately as the intensity of reflection and the two-way travel time from the borehole wall. According to these results, borehole wall texture appears to have little influence on the televiewer logs given in figure 12. In general, areas of low borehole wall reflection amplitude cannot be separated into areas associated with low reflectivity (soft wall rocks or rough borehole wall) and areas of large diameter (where apparently low amplitude reflections result from longer acoustic signal travel paths). Only a few minor examples of this separation can be found in the data. For example, the digitally enhanced BHTV logs do show some minor areas where there are increased acoustic signal travel times but no reflectivity anomalies (A in fig. 13) and minor areas where there are anomalies in amplitude of reflection but not in travel time (B in fig. 13). The data also show that the image enhancement does not remove the equipment-induced noise, which shows up as diagonal bands on the images in figure 13. All openings seen in the BHTV logs are subhorizontal and parallel to bedding in the basalt flows. 


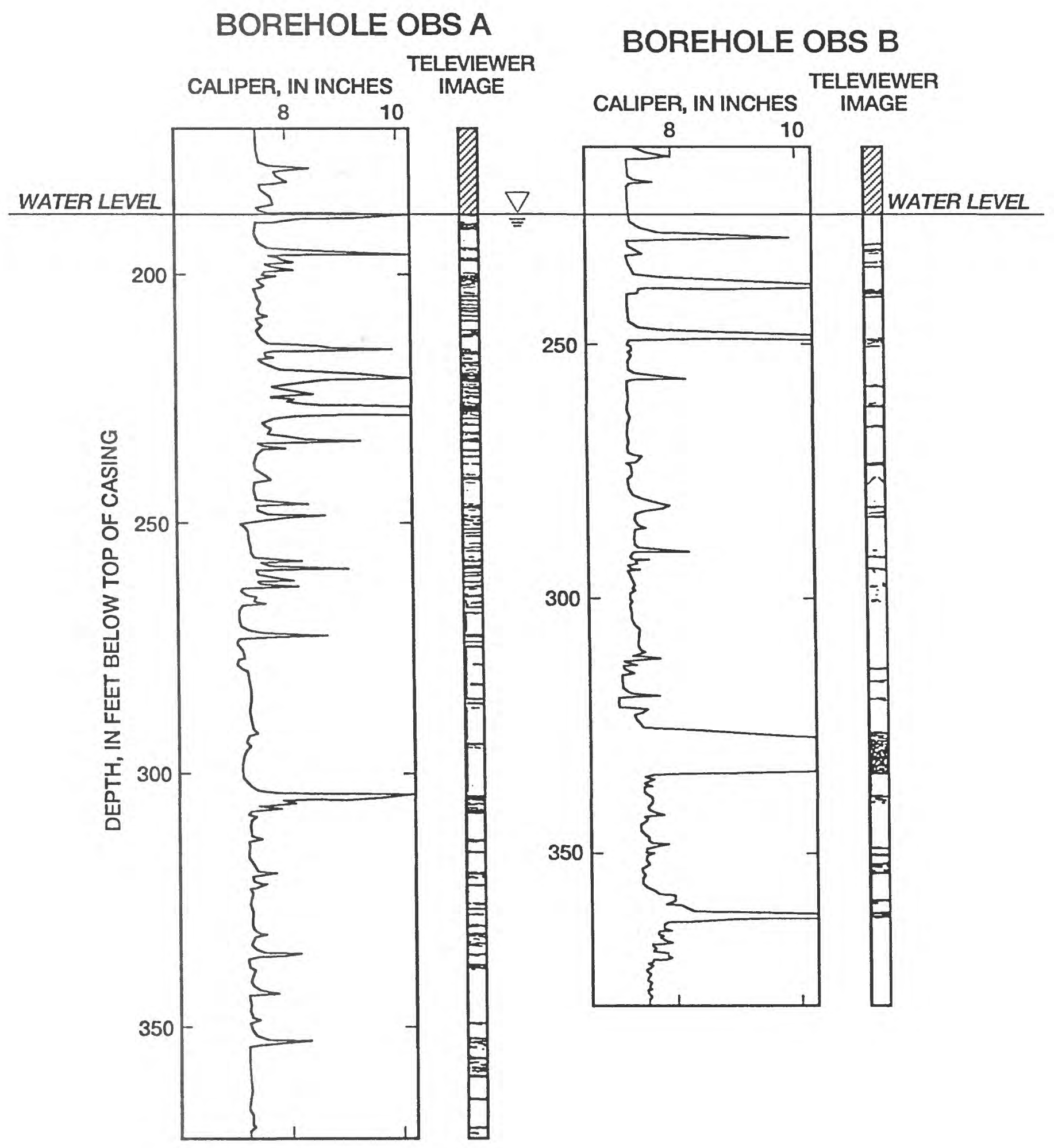

Figure 12. Televiewer image $\log$ interpretations and caliper $\log$ for boreholes $\mathrm{Obs} \mathrm{A}$ and $\mathrm{Obs} \mathrm{B}$. 


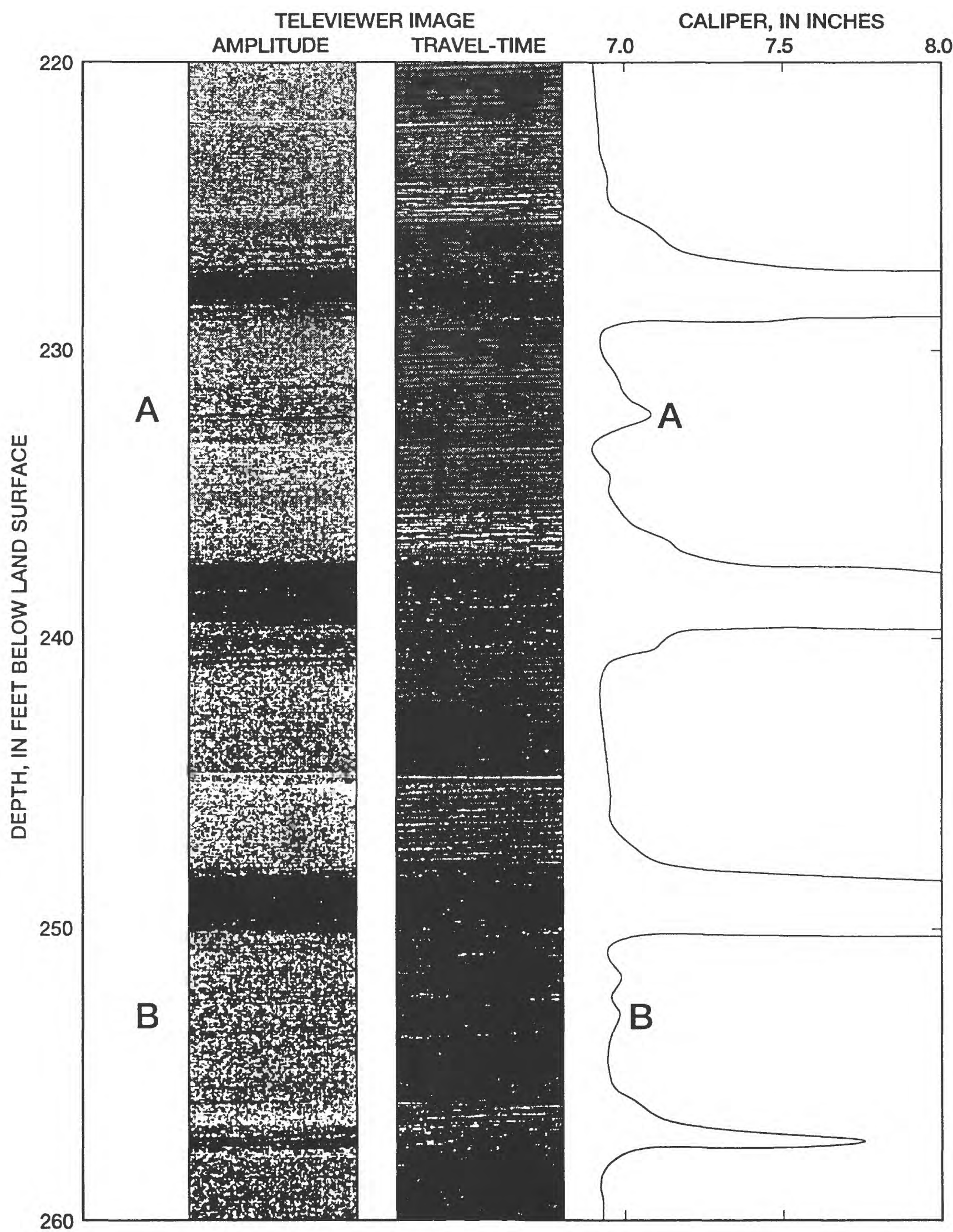

Figure 13. Digitally enhanced interval of televiewer $\log$ for borehole Obs B indicating displays based on both amplitude and travel time of the reflected signal; caliper log shown for comparison. 
The results in figures 12 and 13 indicate that there is no recognizable structure to the openings indicated on the caliper log. These openings represent intervals where unconsolidated clinker zones between aa basalt flows have washed out and eroded during drilling, producing borehole enlargements indicated on the caliper logs (Visher and Mink, 1964; Hunt and others, 1988). The BHTV log data also indicate that there are no features such as fractures or dikes cutting across the flows. Only a single, barely recognizable fracture was identified in the BHTV logs obtained in the four Waipahu boreholes. The diagonal bands of noise present on the processed televiewer logs may obscure a few other minor, closed fractures. However, previous experience in BHTV log interpretation (Paillet and others, 1985) indicates that the diagonal noise bands would not preclude identification of larger, open fractures if they had existed.

\section{Flow under Ambient Hydraulic-Head Conditions in the Waipahu Mahoe Boreholes}

The HPFM measurement series obtained in the Waipahu Mahoe boreholes are summarized in table 2, indicating the mode of HPFM data obtained and the location in table 3 or on figures where these data appear. Fixeddepth mode HPFM data obtained in the Waipahu boreholes under ambient hydraulic-head conditions are listed in table 3. Trolling-mode HPFM data are given in figures 14-20 for the seven Waipahu Mahoe boreholes. The fixeddepth mode HPFM data are given with the date and time of each measurement. Although very few details on the schedule of pumping could be obtained from the Waipahu Mahoe Pump Station and none from other possible production wells in the Waipahu area, the time difference between individual measurements can be used as an indication of the probability that flow conditions had changed between measurements. For measurements that were a few minutes apart, it was unlikely that hydraulic conditions would have changed significantly. When measurements were hours or days apart, it was possible that flow conditions had changed between measurements as pumps were turned on or off, or pumping rates were changed. 
Table 2. Summary of heat-pulse flowmeter logs at the Waipahu wellfield

\begin{tabular}{|c|c|c|c|}
\hline Borehole & Date logged & Conditions & $\begin{array}{c}\text { Location of data in table } 3 \text { or on } \\
\text { figures }\end{array}$ \\
\hline \multirow[t]{7}{*}{ Obs A } & $6-23-87$ & Ambient & 3.1 .1 \\
\hline & $6-24-87$ & Ambient & 3.1 .2 \\
\hline & $6-25-87$ & Ambient & 3.1 .3 \\
\hline & $6-25-87$ & While pumping & 3.1 .4 \\
\hline & $7-13-88$ & Trolling & Figure $14 \mathrm{~A}$ \\
\hline & $7-14-88$ & Ambient & 3.1 .5 \\
\hline & $7-14-88$ & Trolling & Figure $14 \mathrm{~A}, 14 \mathrm{~B}, 14 \mathrm{C}$ \\
\hline \multirow[t]{6}{*}{ Obs B } & $6-30-87$ & Ambient & 3.2 .1 \\
\hline & $7-1-87$ & Ambient & 3.2 .2 \\
\hline & $7-2-87$ & Ambient & 3.2 .3 \\
\hline & 7- 3-87 & Ambient & 3.2 .4 \\
\hline & $7-3-87$ & While pumping & 3.2 .5 \\
\hline & $7-26-88$ & Ambient & 3.2 .6 \\
\hline \multirow[t]{2}{*}{ Obs C } & $7-22-88$ & Ambient & 3.3 .1 \\
\hline & $7-22-88$ & Trolling & Figure 16 \\
\hline \multirow[t]{5}{*}{ Obs D } & $7-12-88$ & Ambient & 3.4 .1 \\
\hline & $7-13-8$ & Ambient & 3.4 .2 \\
\hline & $7-21-88$ & Ambient & 3.4 .3 \\
\hline & $7-21-88$ & While pumping & 3.4 .4 \\
\hline & $7-21-88$ & Trolling while pumping & Figure 17 \\
\hline \multirow[t]{4}{*}{ Obs E } & $7-18-88$ & Ambient & 3.5 .1 \\
\hline & $7-18-88$ & Trolling & Figure 18 \\
\hline & $7-20-88$ & Ambient & 3.5 .2 \\
\hline & $7-20-88$ & While pumping & 3.5 .3 \\
\hline
\end{tabular}


Table 2. Summary of heat-pulse flowmeter logs at the Waipahu wellfield

\begin{tabular}{|c|c|c|c|}
\hline Borehole & Date logged & Conditions & $\begin{array}{c}\text { Location of data in table } 3 \text { or on } \\
\text { figures }\end{array}$ \\
\hline \multirow[t]{6}{*}{ Obs F } & $7-15-88$ & Ambient & 3.6 .1 \\
\hline & $7-15-88$ & Trolling & Figure 19 \\
\hline & $7-19-88$ & Ambient & 3.6 .2 \\
\hline & $7-19-88$ & Below pump & 3.6 .3 \\
\hline & $7-19-88$ & Trolling while pumping & Figure 19 \\
\hline & $7-25-89$ & Ambient & 3.6 .4 \\
\hline \multirow[t]{2}{*}{ Obs H } & $7-26-88$ & Ambient & 3.7 .1 \\
\hline & $7-26-88$ & Trolling & Figure 20 \\
\hline \multirow[t]{9}{*}{ DEEP Obs } & $6-26-87$ & Ambient & 3.8 .1 \\
\hline & $7-27-88$ & Ambient & 3.8 .2 \\
\hline & $7-28-88$ & Ambient & 3.8 .3 \\
\hline & 3- $8-89$ & Ambient & 3.8 .4 \\
\hline & 3- $9-89$ & Ambient & 3.8 .5 \\
\hline & $3-10-89$ & Ambient & 3.8 .6 \\
\hline & $3-13-89$ & Ambient & 3.8 .7 \\
\hline & $3-13-89$ & Trolling & Figure 22 \\
\hline & $3-14-89$ & Ambient & 3.8 .8 \\
\hline
\end{tabular}


Table 3. Heat-pulse flowmeter data from the Waipahu boreholes [gal/min, gallons per minute]

\begin{tabular}{|c|c|c|c|c|c|}
\hline $\begin{array}{l}\text { Depth }^{1} \\
\text { (feet) }\end{array}$ & Packer $^{2}$ & $\begin{array}{l}\text { Pulse time }{ }^{3} \\
\text { (seconds) }\end{array}$ & $\begin{array}{l}\text { Inverse time } \\
\text { (1/second) }\end{array}$ & $\begin{array}{c}\text { Flow }^{4,5} \\
\text { (gal/min) }\end{array}$ & $\begin{array}{c}\text { Time } \\
\text { (hour:minute) }\end{array}$ \\
\hline \multicolumn{6}{|c|}{ 3.1 DATA FOR BOREHOLE OBS A } \\
\hline \multicolumn{6}{|c|}{ 3.1.1 6-23-87 Ambient tlow data } \\
\hline 230 & $\mathrm{X}$ & -3.5 & -0.29 & -0.26 & $14: 45$ \\
\hline 370 & $\mathrm{O}$ & 6.4 & 0.16 & 3.30 & $15: 22$ \\
\hline 390 & $\mathrm{O}$ & 9.5 & 0.11 & 2.20 & $15: 30$ \\
\hline 350 & $\mathrm{O}$ & 6.1 & 0.16 & 3.30 & $15: 36$ \\
\hline 330 & $\mathrm{O}$ & 11.3 & 0.09 & 1.80 & $15: 43$ \\
\hline 312 & $\mathrm{O}$ & 16.0 & 0.06 & 1.30 & $15: 53$ \\
\hline 300 & O & 20.0 & 0.05 & 1.00 & $16: 07$ \\
\hline 380 & $\mathrm{O}$ & 30.0 & 0.03 & 0.70 & $16: 20$ \\
\hline 280 & $\mathrm{X}$ & 2.2 & 0.45 & 0.53 & $16: 30$ \\
\hline 260 & $\mathrm{X}$ & -18.0 & -0.05 & -0.04 & $16: 59$ \\
\hline 270 & $\mathrm{X}$ & 6.0 & 0.17 & 0.12 & $17: 08$ \\
\hline 250 & $x$ & -4.5 & -0.22 & -0.18 & $17: 20$ \\
\hline 215 & $X$ & 1.3 & 0.75 & 1.27 & $17: 35$ \\
\hline 197 & $x$ & 3.4 & 0.29 & 0.28 & $17: 45$ \\
\hline 191 & $x$ & $\mathrm{NF}^{6}$ & NF & NF & $17: 52$ \\
\hline 193 & $x$ & -40.0 & -0.015 & -0.03 & $18: 02$ \\
\hline 195 & $X$ & 5.3 & 0.19 & 0.15 & $18: 14$ \\
\hline 227 & $x$ & NF & NF & $\mathrm{NF}$ & $18: 27$ \\
\hline 218 & $X$ & 1.4 & 0.70 & 1.17 & $18: 42$ \\
\hline 218 & O & 14.5 & 0.07 & 1.40 & $18: 46$ \\
\hline \multicolumn{6}{|c|}{ 3.1.2 6-24-87 Ambient flow data } \\
\hline 230 & $\mathrm{O}$ & -25.00 & -0.04 & -1.00 & $15: 44$ \\
\hline 230 & $x$ & -1.6 & -0.63 & -0.95 & $15: 59$ \\
\hline 240 & $\mathrm{O}$ & -70.9 & 0.01 & -0.40 & $16: 05$ \\
\hline 240 & $\mathrm{x}$ & -3.3 & -0.30 & -0.28 & $16: 19$ \\
\hline 350 & $\mathrm{O}$ & 7.0 & 0.14 & 2.90 & $16: 46$ \\
\hline 360 & $\mathrm{O}$ & 7.4 & 0.14 & 2.70 & $16: 54$ \\
\hline 340 & $\mathrm{O}$ & 11.1 & 0.09 & 1.90 & $17: 06$ \\
\hline 330 & $\mathrm{O}$ & 25.7 & 0.04 & 0.80 & $17: 11$ \\
\hline 250 & $\mathrm{O}$ & -20.0 & 0.05 & -1.30 & $17: 25$ \\
\hline 250 & $x$ & -1.7 & -0.59 & -0.85 & $17: 39$ \\
\hline 215 & $x$ & 1.5 & 0.67 & 1.00 & $17: 48$ \\
\hline 210 & $\mathrm{x}$ & 1.4 & 0.70 & 1.14 & $17: 53$ \\
\hline 202 & $x$ & 1.4 & 0.73 & 1.20 & $18: 02$ \\
\hline 197 & $\mathrm{x}$ & 4.0 & 0.25 & 0.22 & $18: 07$ \\
\hline \multicolumn{6}{|c|}{ 3.1.3 6-25-87 Ambient flow data } \\
\hline 210 & $\mathrm{O}$ & -25.0 & -0.04 & -1.10 & $09: 31$ \\
\hline 210 & $x$ & -4.0 & -.25 & -0.21 & $09: 43$ \\
\hline 250 & $\mathrm{O}$ & 5.8 & 0.17 & 3.60 & $09: 53$ \\
\hline 300 & $\mathrm{O}$ & 5.5 & 0.18 & 3.80 & $09: 58$ \\
\hline 350 & $\mathrm{O}$ & 3.5 & 0.29 & 5.90 & $10: 02$ \\
\hline 235 & $\mathrm{O}$ & 10.7 & 0.09 & 1.90 & $10: 12$ \\
\hline
\end{tabular}


Table 3. Heat-pulse flowmeter data from the Waipahu boreholes [gal/min, gallons per minute]

\begin{tabular}{|c|c|c|c|c|c|}
\hline $\begin{array}{l}\text { Depth' } \\
\text { (feet) }\end{array}$ & Packer $^{2}$ & $\begin{array}{l}\text { Pulse time } \\
\text { (seconds) }\end{array}$ & $\begin{array}{l}\text { Inverse time } \\
\text { (1/second) }\end{array}$ & $\begin{array}{l}\text { Flow }^{4,5} \\
\text { (gal/min) }\end{array}$ & $\begin{array}{c}\text { Time } \\
\text { (hour:minute) }\end{array}$ \\
\hline 250 & $\mathrm{O}$ & 11.2 & 0.09 & 1.80 & $10: 22$ \\
\hline 210 & $\mathrm{x}$ & 3.4 & 0.29 & 0.28 & $10: 29$ \\
\hline 210 & $\mathrm{x}$ & 1.5 & 0.67 & 1.02 & $10: 34$ \\
\hline 300 & $\mathrm{O}$ & 6.3 & 0.16 & 3.30 & $10: 46$ \\
\hline 300 & $\mathrm{O}$ & 5.9 & 0.17 & 3.50 & $11: 07$ \\
\hline 350 & $\mathrm{O}$ & 2.9 & 0.35 & 7.10 & $11: 20$ \\
\hline 250 & $\mathrm{O}$ & 13.9 & 0.07 & 1.50 & $11: 35$ \\
\hline 250 & $\mathrm{O}$ & 3.7 & 0.27 & 5.60 & $11: 39$ \\
\hline 235 & $\mathrm{O}$ & 17.7 & 0.06 & 1.20 & $11: 44$ \\
\hline 210 & $\mathrm{x}$ & 3.0 & 0.33 & 0.34 & $11: 54$ \\
\hline 210 & $\mathrm{X}$ & 1.2 & 0.81 & 1.38 & $12: 02$ \\
\hline 210 & $\mathrm{X}$ & 1.8 & 0.56 & 0.78 & $12: 09$ \\
\hline 250 & $X$ & 2.8 & 0.36 & 0.37 & $15: 12$ \\
\hline 300 & $\mathrm{O}$ & 14.0 & 0.07 & 1.50 & $15: 24$ \\
\hline 350 & $\mathrm{O}$ & 6.1 & 0.16 & 3.40 & $15: 31$ \\
\hline 250 & $\mathrm{O}$ & -20.0 & -0.05 & -1.30 & $15: 47$ \\
\hline 250 & $X$ & -2.4 & -0.41 & -0.45 & $15: 59$ \\
\hline 235 & $\mathrm{O}$ & -19.0 & -0.05 & -1.40 & $16: 14$ \\
\hline 210 & O & 11.2 & 0.09 & 1.80 & $16: 31$ \\
\hline \multicolumn{6}{|c|}{$3.1 .46-25-7$ log whik ruming, $14.18 \mathrm{gal} / \mathrm{min}$ Storli get $16: 42$} \\
\hline 250 & $\mathrm{O}$ & 5.3 & 0.19 & 3.90 & $16: 53$ \\
\hline 235 & $\mathrm{O}$ & 5.3 & 0.19 & 3.90 & $17: 02$ \\
\hline 210 & $\mathrm{O}$ & 1.2 & 0.81 & 16.70 & $17: 13$ \\
\hline 215 & 0 & 1.6 & 0.64 & 13.00 & $17: 22$ \\
\hline 218 & $\mathrm{O}$ & 1.4 & 0.71 & 14.70 & $17: 25$ \\
\hline 222 & $\mathrm{O}$ & $\mathrm{TF}^{7}$ & $\mathrm{TF}$ & $\mathrm{TF}$ & $17: 29$ \\
\hline 225 & $\mathrm{O}$ & $\mathrm{TF}$ & $\mathrm{TF}$ & TF & $17: 31$ \\
\hline 220 & $\mathrm{O}$ & 1.4 & 0.70 & 14.40 & $17: 38$ \\
\hline 230 & $\mathrm{O}$ & 5.8 & 0.17 & 3.50 & $17: 44$ \\
\hline 235 & $\mathrm{O}$ & 6.6 & 0.15 & 3.10 & $17: 51$ \\
\hline 250 & $\mathrm{O}$ & 5.7 & 0.18 & 3.60 & $17: 57$ \\
\hline 300 & $\mathrm{O}$ & 4.4 & 0.23 & 4.60 & 18:14 \\
\hline 350 & $\mathrm{O}$ & 6.5 & 0.15 & 3.20 & $18: 25$ \\
\hline 390 & $\mathrm{O}$ & 10.4 & 0.10 & 2.00 & $18: 33$ \\
\hline 235 & $\mathrm{O}$ & 7.7 & 0.13 & 2.70 & 19:02 \\
\hline \multicolumn{6}{|c|}{ Increase pumping rate to $27.8 \mathrm{gal} / \mathrm{min}$ at $19: 04$} \\
\hline 235 & $\mathrm{O}$ & 2.38 & 0.42 & 8.70 & 19:06 \\
\hline 300 & $\mathrm{O}$ & 2.73 & 0.37 & 7.60 & $19: 22$ \\
\hline 350 & O & 4.68 & 0.21 & 4.40 & $19: 27$ \\
\hline \multicolumn{6}{|c|}{ 3.1.5 7-14-88 Ambient flow data } \\
\hline 200 & $\mathrm{O}$ & -1.5 & -0.67 & -16.00 & $11: 54$ \\
\hline 190 & 0 & NF & NF & NF & 12:05 \\
\hline 190 & $x$ & -7.0 & -0.14 & 0.10 & $12: 11$ \\
\hline 200 & $\mathrm{O}$ & -1.4 & -0.73 & -22.00 & $14: 23$ \\
\hline 200 & $\mathrm{X}$ & TF & $\mathrm{TF}$ & TF & $14: 30$ \\
\hline
\end{tabular}


Table 3. Heat-pulse flowmeter data from the Waipahu boreholes [gal/min, gallons per minute]

\begin{tabular}{|c|c|c|c|c|c|}
\hline $\begin{array}{l}\text { Depth }{ }^{1} \\
\text { (feet) }\end{array}$ & Packer ${ }^{2}$ & $\begin{array}{l}\text { Pulse time }{ }^{3} \\
\text { (seconds) }\end{array}$ & $\begin{array}{l}\text { Inverse time } \\
\text { (1/second) }\end{array}$ & $\begin{array}{c}\text { Flow }{ }^{4,5} \\
\text { (gal/min) }\end{array}$ & $\begin{array}{c}\text { Time } \\
\text { (hour:minute) }\end{array}$ \\
\hline 210 & $\mathrm{O}$ & -1.3 & -0.80 & -22.00 & $14: 37$ \\
\hline 220 & O & -1.3 & -0.78 & -21.00 & $14: 48$ \\
\hline 230 & $\mathrm{O}$ & 3.2 & 0.31 & 6.40 & $15: 00$ \\
\hline 230 & $\mathrm{O}$ & 4.3 & 0.23 & 4.60 & $15: 02$ \\
\hline 240 & $\mathrm{O}$ & 6.2 & 0.16 & 3.30 & 15:06 \\
\hline 280 & 0 & 3.2 & 0.32 & 6.40 & $15: 12$ \\
\hline 300 & $\mathrm{O}$ & 3.2 & 0.32 & 6.40 & $15: 18$ \\
\hline 305 & $\mathrm{O}$ & 1.4 & 0.71 & 15.90 & $15: 22$ \\
\hline 310 & 0 & 1.3 & 0.75 & 16.90 & $15: 26$ \\
\hline 315 & $\mathrm{O}$ & 1.4 & 0.74 & 16.60 & $15: 30$ \\
\hline 330 & $\mathrm{O}$ & 1.3 & 0.77 & 17.50 & $15: 37$ \\
\hline 360 & $\mathrm{O}$ & 1.5 & 0.67 & 14.70 & $15: 42$ \\
\hline 375 & $\mathrm{O}$ & 1.6 & 0.63 & 13.70 & $15: 49$ \\
\hline \multicolumn{2}{|c|}{ 3.2.1 6-30-87 Ambient How data } & & & & \\
\hline 232 & $\mathrm{O}$ & -15.0 & -0.07 & -1.80 & 17:09 \\
\hline 260 & $\mathrm{O}$ & -15.0 & -0.07 & -1.80 & $17: 31$ \\
\hline 306 & $\mathrm{O}$ & -20.0 & -0.05 & -1.40 & $17: 47$ \\
\hline 346 & $\mathrm{O}$ & 7.8 & 0.13 & 2.60 & $18: 03$ \\
\hline 380 & $\mathrm{O}$ & 9.1 & 0.11 & 2.20 & 18:13 \\
\hline 410 & $\mathrm{O}$ & 17.8 & 0.06 & 1.10 & $18: 22$ \\
\hline 445 & $\mathrm{O}$ & 27.3 & 0.04 & 0.70 & $18: 33$ \\
\hline 355 & $\mathrm{O}$ & 6.1 & 0.16 & 3.10 & $18: 42$ \\
\hline 318 & $\mathrm{O}$ & -11.8 & -0.09 & -2.30 & $18: 53$ \\
\hline 306 & $\mathrm{O}$ & -14.8 & -0.07 & -1.80 & 19:05 \\
\hline 285 & $\mathrm{O}$ & -21.3 & -0.05 & -1.30 & $19: 16$ \\
\hline 242 & $\mathrm{O}$ & -14.8 & -0.07 & -1.40 & $19: 30$ \\
\hline 232 & $\mathrm{O}$ & -22.0 & -0.05 & -1.20 & $19: 42$ \\
\hline \multicolumn{6}{|c|}{$3.2 .27-1-87$ Ambient flow data } \\
\hline 318 & $\mathrm{O}$ & -9.1 & -0.11 & -2.90 & $12: 44$ \\
\hline 355 & 0 & 8.0 & 0.13 & 3.10 & $13: 20$ \\
\hline 346 & $\mathrm{O}$ & 9.0 & 0.11 & 2.70 & $14: 37$ \\
\hline 318 & $\mathrm{O}$ & -14.1 & -0.07 & -1.90 & $15: 39$ \\
\hline 346 & $\mathrm{O}$ & 9.5 & 0.10 & 2.30 & $16: 28$ \\
\hline \multicolumn{6}{|c|}{$3.2 .37-2-87$ Ambient flow data } \\
\hline 318 & $\mathrm{O}$ & -9.0 & -0.11 & -2.90 & 08:06 \\
\hline 346 & $\mathrm{O}$ & 9.0 & 0.11 & 2.70 & 09:06 \\
\hline 318 & 0 & -15.0 & -0.07 & -1.90 & $10: 17$ \\
\hline 346 & 0 & 9.0 & 0.11 & 2.70 & 11:09 \\
\hline 318 & 0 & -11.5 & -0.09 & -2.40 & $11: 34$ \\
\hline \multicolumn{6}{|c|}{ 3.2.4 7-3-87 Ambient flow data } \\
\hline 242 & $\mathrm{O}$ & NF & NF & NF & $08: 57$ \\
\hline 260 & $\mathrm{O}$ & NF & NF & NF & $09: 13$ \\
\hline 285 & $\mathrm{O}$ & -25.0 & -0.04 & -1.00 & $09: 26$ \\
\hline 306 & $\mathrm{O}$ & -14.6 & -0.07 & -1.80 & $09: 52$ \\
\hline 318 & $\mathrm{O}$ & -17.6 & -0.06 & -1.20 & $10: 10$ \\
\hline
\end{tabular}


Table 3. Heat-pulse flowmeter data from the Waipahu boreholes [gal/min, gallons per minute]

\begin{tabular}{|c|c|c|c|c|c|}
\hline $\begin{array}{l}\text { Depth' } \\
\text { (feet) }\end{array}$ & Packer $^{2}$ & $\begin{array}{l}\text { Pulse time } \\
\text { (seconds) }\end{array}$ & $\begin{array}{l}\text { Inverse time } \\
\text { (1/second) }\end{array}$ & $\begin{array}{c}\text { Flow }{ }^{4,5} \\
\text { (gal/min) }\end{array}$ & $\begin{array}{c}\text { Time } \\
\text { (hour:minute) }\end{array}$ \\
\hline 346 & $\mathrm{O}$ & 8.3 & 0.12 & 2.50 & $10: 26$ \\
\hline 355 & $\mathrm{O}$ & 8.5 & 0.12 & 2.40 & $10: 38$ \\
\hline 380 & $\mathrm{O}$ & 13.5 & 0.07 & 1.50 & $10: 53$ \\
\hline 410 & $\mathrm{O}$ & 35.0 & 0.03 & 0.60 & $11: 14$ \\
\hline 445 & $\mathrm{O}$ & 40.6 & 0.03 & 0.50 & $11: 36$ \\
\hline \multicolumn{6}{|c|}{ 3.2.5 7-3-87 Log while pumping at $26.9 \mathrm{gal} / \mathrm{min}$ starting at $12: 40$} \\
\hline 445 & $\mathrm{O}$ & 37.8 & 0.03 & 0.60 & $14: 07$ \\
\hline 410 & $\mathrm{O}$ & 22.4 & 0.05 & 0.90 & $14: 24$ \\
\hline 380 & $\mathrm{O}$ & 7.6 & 0.13 & 2.70 & $14: 46$ \\
\hline 355 & $\mathrm{O}$ & 4.9 & 0.20 & 4.20 & $14: 52$ \\
\hline 346 & $\mathrm{O}$ & 5.4 & 0.19 & 3.80 & $15: 02$ \\
\hline 318 & $\mathrm{O}$ & 1.7 & 0.57 & 11.80 & $15: 18$ \\
\hline 306 & $\mathrm{O}$ & 1.7 & 0.59 & 12.10 & $15: 30$ \\
\hline 285 & $\mathrm{O}$ & 1.5 & 0.65 & 13.40 & $15: 48$ \\
\hline 260 & 0 & 1.4 & 0.73 & 15.00 & $15: 58$ \\
\hline 242 & 0 & 1.2 & 0.80 & 21.00 & $16: 04$ \\
\hline \multicolumn{6}{|c|}{ 3.2.6 7-26-88 Ambient flow data } \\
\hline 223 & $\mathrm{X}$ & -2.6 & -0.38 & -0.41 & $10: 28$ \\
\hline 243 & $x$ & -1.6 & -0.64 & -0.87 & $10: 44$ \\
\hline 260 & $\mathrm{X}$ & -1.7 & -0.59 & -0.78 & $10: 56$ \\
\hline 278 & $x$ & -1.5 & -0.67 & -0.95 & $11: 04$ \\
\hline 300 & $\mathrm{X}$ & -1.3 & -0.77 & -1.20 & $11: 11$ \\
\hline 300 & $\mathrm{O}$ & -8.0 & -0.12 & -3.40 & $11: 17$ \\
\hline 320 & $x$ & -1.4 & -0.71 & -1.10 & $11: 24$ \\
\hline 320 & $\mathrm{O}$ & -10.0 & -0.10 & -2.70 & $11: 28$ \\
\hline 340 & $\mathrm{O}$ & 3.7 & 0.27 & 5.50 & $11: 41$ \\
\hline 355 & $\mathrm{O}$ & 3.3 & 0.30 & 6.10 & $11: 50$ \\
\hline 375 & $\mathrm{O}$ & 4.2 & 0.24 & 4.90 & $11: 58$ \\
\hline 390 & $\mathrm{O}$ & 6.2 & 0.16 & 3.30 & $12: 04$ \\
\hline 390 & $x$ & 0.9 & 1.10 & 2.60 & $12: 07$ \\
\hline 410 & $\mathrm{O}$ & 15.0 & 0.07 & 1.30 & $12: 20$ \\
\hline 410 & $x$ & 1.8 & 0.56 & 0.73 & $12: 26$ \\
\hline 419 & $\mathrm{O}$ & 25.0 & 0.04 & 0.80 & $12: 40$ \\
\hline 419 & $\mathrm{X}$ & 4.3 & 0.23 & 0.20 & $12: 45$ \\
\hline \multicolumn{6}{|c|}{ 3.3 DATA FOR BOREHOLE OBS C } \\
\hline \multicolumn{6}{|c|}{ 3.3.1 7-22-88 Ambient flow data } \\
\hline 200 & 0 & 1.5 & 0.95 & 25.30 & $10: 58$ \\
\hline 220 & 0 & $\mathrm{TF}$ & $\mathrm{TF}$ & $\mathrm{TF}$ & 11:07 \\
\hline 220 & $x$ & $\mathrm{TF}$ & $\mathrm{TF}$ & $\mathrm{TF}$ & $11: 12$ \\
\hline 237 & $\mathrm{O}$ & 1.0 & 1.00 & 26.00 & $11: 24$ \\
\hline 260 & 0 & $\mathrm{TF}$ & $\mathrm{TF}$ & TF & $11: 34$ \\
\hline 250 & $\mathrm{O}$ & $\mathrm{TF}$ & 0.76 & 25.00 & $11: 40$ \\
\hline 290 & $\mathrm{O}$ & 2.0 & 0.49 & 10.40 & $11: 48$ \\
\hline 310 & $\mathrm{O}$ & 2.3 & 0.44 & 9.50 & $11: 55$ \\
\hline 345 & $\mathrm{O}$ & 2.6 & 0.38 & 8.60 & $11: 58$ \\
\hline 370 & $\mathrm{O}$ & 3.6 & 0.28 & 5.70 & $12: 02$ \\
\hline
\end{tabular}




\section{CONTENTS}

Abstract

Introduction

Description of geophysical logs

Conventional geophysical logs

Acoustic borehole televiewer (BHTV) logs.........................................................................................................

Heat-pulse flowmeter (HPFM) logs ............................................................................................................ 5

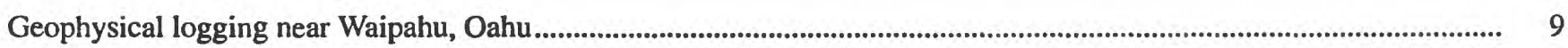

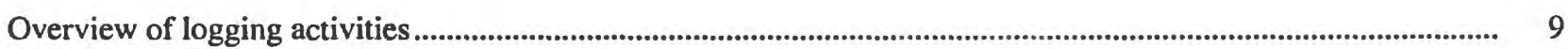

Conventional logs for the Waipahu Mahoe boreholes ...................................................................................... 13

Acoustic borehole televiewer logs for the Waipahu Mahoe boreholes............................................................. 21

Flow under ambient hydraulic-head conditions in the Waipahu Mahoe boreholes ................................................ 24

Flow in the Waipahu Mahoe boreholes during borehole pumping ................................................................. 50

Logs from the Waipio Deep Observation borehole............................................................................................ 50

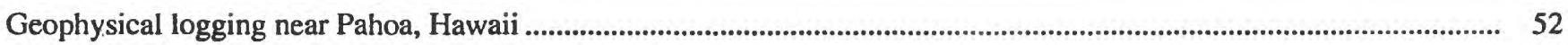

Overview of logging activities at the Pahoa Site .................................................................................... 52

Conventional geophysical logs in borehole SOH-2 ................................................................................................ 54

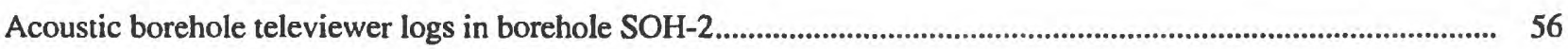

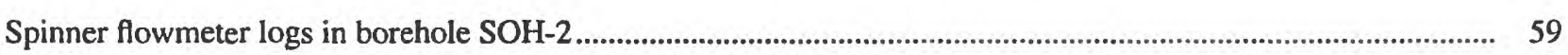

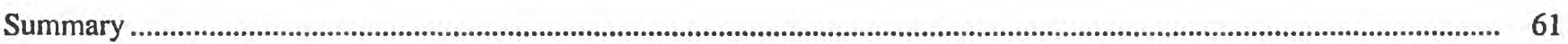

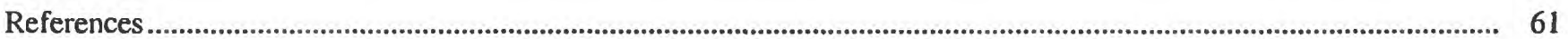


Table 3. Heat-pulse flowmeter data from the Waipahu boreholes [gal/min, gallons per minute]

\begin{tabular}{|c|c|c|c|c|c|}
\hline $\begin{array}{l}\text { Depth } \\
\text { (feet) }\end{array}$ & Packer $^{2}$ & $\begin{array}{l}\text { Pulse time } \\
\text { (seconds) }\end{array}$ & $\begin{array}{l}\text { Inverse time } \\
\text { (1/second) }\end{array}$ & $\begin{array}{l}\text { Flow }^{4,5} \\
\text { (gal/min) }\end{array}$ & $\begin{array}{c}\text { Time } \\
\text { (hour:minute) }\end{array}$ \\
\hline 388 & $\mathrm{O}$ & 4.4 & 0.23 & 4.70 & $12: 08$ \\
\hline 410 & $\mathrm{O}$ & 25.0 & 0.04 & 0.80 & $12: 16$ \\
\hline \multicolumn{6}{|c|}{ 3.4 DATA FOR BOREHOLE OBS D } \\
\hline \multicolumn{6}{|c|}{3.4 .1 7-12-88 Ambient flow data } \\
\hline 160 & $\mathrm{O}$ & 9.0 & 0.11 & 2.30 & $16: 49$ \\
\hline 160 & $X$ & 1.9 & 0.53 & 0.71 & $16: 58$ \\
\hline \multicolumn{6}{|c|}{ 3.4.2 7-13-88 Ambient flow data } \\
\hline 190 & $\mathrm{X}$ & -4.0 & -0.25 & -0.21 & $10: 50$ \\
\hline 230 & $x$ & -2.0 & -0.50 & -0.61 & $11: 18$ \\
\hline 275 & $\mathrm{X}$ & -2.4 & -0.42 & -0.46 & $11: 28$ \\
\hline 330 & $\mathrm{X}$ & 3.5 & 0.29 & 0.27 & $11: 36$ \\
\hline 344 & $x$ & 4.6 & 0.22 & 0.18 & $11: 43$ \\
\hline 320 & $x$ & 3.1 & 0.31 & 0.30 & $11: 56$ \\
\hline 310 & $x$ & 3.5 & 0.28 & 0.27 & $12: 05$ \\
\hline 300 & $x$ & -4.6 & -0.22 & -0.17 & $12: 14$ \\
\hline 300 & $X$ & -5.6 & -0.18 & -0.13 & $12: 18$ \\
\hline 290 & $X$ & -3.5 & -0.29 & -0.25 & $12: 25$ \\
\hline 280 & $X$ & -3.2 & -0.30 & -0.28 & $12: 34$ \\
\hline 270 & $X$ & -2.5 & -0.40 & -0.43 & $12: 40$ \\
\hline 260 & $x$ & -2.4 & -0.42 & -0.47 & $12: 59$ \\
\hline 248 & $x$ & -2.3 & -0.43 & -0.48 & $13: 04$ \\
\hline 240 & $x$ & -2.3 & -0.43 & -0.50 & $13: 18$ \\
\hline 230 & $x$ & -2.3 & -0.43 & -0.49 & $13: 23$ \\
\hline 230 & $X$ & -2.7 & -0.34 & -0.34 & $13: 26$ \\
\hline 190 & $x$ & -6.0 & -0.17 & -0.12 & $13: 33$ \\
\hline 190 & $x$ & -9.5 & -0.11 & -0.07 & $13: 36$ \\
\hline 190 & $x$ & -9.0 & -0.11 & -0.07 & $13: 38$ \\
\hline 160 & $X$ & 2.3 & 0.43 & 0.53 & $13: 47$ \\
\hline 140 & $X$ & -9.5 & -0.11 & -0.07 & $13: 58$ \\
\hline 150 & $x$ & 2.4 & 0.42 & 0.48 & $14: 03$ \\
\hline \multicolumn{6}{|c|}{ 3.4.3 7-21-88 Ambient flow data } \\
\hline 135 & $x$ & -7.0 & -0.14 & -0.10 & $10: 10$ \\
\hline 150 & $\mathrm{X}$ & 7.2 & 0.14 & 0.10 & $10: 16$ \\
\hline 166 & $\mathrm{O}$ & 2.8 & 0.04 & 0.70 & $10: 24$ \\
\hline 230 & $X$ & -2.0 & -0.51 & -0.52 & $10: 48$ \\
\hline 248 & $\mathrm{X}$ & -2.2 & -0.45 & -0.44 & $10: 54$ \\
\hline 260 & $x$ & -1.6 & -0.61 & -0.68 & $11: 05$ \\
\hline 275 & $x$ & -1.9 & -0.52 & -0.53 & $11: 11$ \\
\hline 300 & $x$ & -3.1 & -0.32 & -0.27 & $11: 16$ \\
\hline 320 & $x$ & 3.3 & 0.30 & 0.28 & $11: 20$ \\
\hline 344 & $\mathrm{X}$ & 5.3 & 0.19 & 0.15 & $11: 26$ \\
\hline 200 & $x$ & -2.5 & -0.40 & -0.38 & $11: 35$ \\
\hline 180 & $x$ & -2.5 & -0.40 & -0.37 & $11: 43$ \\
\hline 160 & $\mathrm{X}$ & 2.5 & 0.41 & 0.47 & $11: 51$ \\
\hline \multicolumn{6}{|c|}{ 3.4.4 7-21-88 L.og while pumping-27.0 gal $/ \mathrm{min}$ starting at 12:00 } \\
\hline 344 & $\mathrm{X}$ & 3.0 & 0.33 & 0.33 & $12: 24$ \\
\hline
\end{tabular}


Table 3. Heat-pulse flowmeter data from the Waipahu boreholes [gal/min, gallons per minute]

\begin{tabular}{|c|c|c|c|c|c|}
\hline $\begin{array}{l}\text { Depth }^{1} \\
\text { (feet) }\end{array}$ & Packer $^{2}$ & $\begin{array}{l}\text { Pulse time }{ }^{3} \\
\text { (seconds) }\end{array}$ & $\begin{array}{l}\text { Inverse time } \\
\text { (1/second) }\end{array}$ & $\begin{array}{l}\text { Flow }^{4,5} \\
\text { (gal/min) }\end{array}$ & $\begin{array}{c}\text { Time } \\
\text { (hour:minute) }\end{array}$ \\
\hline 150 & 0 & 1.2 & 0.87 & 21.20 & $12: 59$ \\
\hline 150 & 0 & 0.9 & 1.11 & 30.00 & $13: 00$ \\
\hline 166 & $\mathrm{O}$ & $\mathrm{N}$ & $\mathrm{N}$ & $\mathrm{N}$ & $13: 03$ \\
\hline 180 & $\mathrm{O}$ & 1.2 & 0.86 & 21.00 & $13: 07$ \\
\hline 200 & $\mathrm{O}$ & 1.1 & 0.90 & 22.00 & $13: 12$ \\
\hline 230 & 0 & 1.4 & 0.73 & 17.00 & $13: 14$ \\
\hline 248 & 0 & $N$ & $\mathrm{~N}$ & $\mathrm{~N}$ & $13: 19$ \\
\hline 260 & $\mathrm{O}$ & 11.0 & 0.09 & 1.70 & $13: 25$ \\
\hline 275 & 0 & 32.0 & 0.03 & 0.60 & $13: 28$ \\
\hline 275 & $\mathrm{X}$ & 1.7 & 0.60 & 0.87 & $13: 32$ \\
\hline 300 & $X$ & 1.6 & 0.63 & 0.96 & $13: 38$ \\
\hline 320 & $\mathrm{X}$ & 1.5 & 0.66 & 1.03 & $13: 44$ \\
\hline 344 & $\mathrm{X}$ & 3.0 & 0.33 & 0.33 & $13: 49$ \\
\hline \multicolumn{6}{|c|}{ Pump flow adjusted to $9.2 \mathrm{gal} / \mathrm{min}$ at $13: 55$} \\
\hline 200 & $\mathrm{O}$ & 3.2 & 0.31 & 6.60 & $14: 00$ \\
\hline 135 & $\mathrm{O}$ & 1.9 & 0.52 & 11.40 & $14: 03$ \\
\hline 150 & $\mathrm{O}$ & 2.2 & 0.46 & 9.90 & $14: 06$ \\
\hline 166 & $\mathrm{O}$ & 2.2 & 0.45 & 9.70 & 14:09 \\
\hline 180 & $\mathrm{O}$ & 3.5 & 0.29 & 6.00 & $14: 12$ \\
\hline 200 & $\mathrm{O}$ & 3.4 & 0.29 & 6.20 & $14: 15$ \\
\hline \multicolumn{6}{|c|}{ 3.5 DATA FOR BOREHOLE OBS E } \\
\hline \multicolumn{6}{|c|}{ 3.5.1 7-18-88 Ambient flow data } \\
\hline 200 & $\mathrm{O}$ & 9.0 & 0.11 & 2.20 & $15: 10$ \\
\hline 200 & $\mathrm{X}$ & 1.7 & 0.59 & 0.83 & $15: 14$ \\
\hline 185 & $\mathrm{O}$ & 20.0 & 0.05 & 1.00 & $15: 20$ \\
\hline 185 & $x$ & 1.9 & 0.51 & 0.65 & $15: 25$ \\
\hline 220 & $\mathrm{O}$ & -15.0 & -0.07 & -1.80 & $15: 40$ \\
\hline 220 & $x$ & -1.5 & -0.67 & -0.95 & $15: 45$ \\
\hline 235 & $\mathrm{O}$ & 6.0 & 0.17 & 3.50 & $15: 51$ \\
\hline 235 & $x$ & 1.5 & 0.67 & 1.03 & $15: 55$ \\
\hline 270 & 0 & 2.7 & 0.37 & 7.40 & $16: 01$ \\
\hline 270 & $X$ & $\mathrm{TF}$ & $\mathrm{TF}$ & $\mathrm{TF}$ & $16: 06$ \\
\hline 310 & $\mathrm{O}$ & 1.4 & 0.71 & 16.40 & $16: 13$ \\
\hline 345 & 0 & 1.0 & 1.00 & 23.20 & $16: 21$ \\
\hline 365 & 0 & 1.1 & 0.91 & 23.20 & $16: 26$ \\
\hline 390 & O & 1.1 & 0.91 & 23.20 & $16: 32$ \\
\hline 415 & $\mathrm{O}$ & 2.7 & 0.37 & 7.60 & $16: 36$ \\
\hline 425 & $\mathrm{O}$ & 3.4 & 0.29 & 5.30 & $16: 40$ \\
\hline 433 & $\mathrm{O}$ & 15.5 & 0.06 & 1.40 & $16: 47$ \\
\hline \multicolumn{6}{|c|}{3.5 .2 7-20-88 Ambient flow data } \\
\hline 195 & $\mathrm{O}$ & -50.0 & -0.02 & -0.40 & $09: 45$ \\
\hline 195 & $x$ & -11.5 & -0.08 & -0.06 & $09: 56$ \\
\hline 200 & $\mathrm{O}$ & NF & NF & NF & $10: 05$ \\
\hline 200 & $x$ & NF & NF & NF & $10: 10$ \\
\hline 210 & $\mathrm{O}$ & NF & $\mathrm{NF}$ & NF & $10: 16$ \\
\hline 210 & $\mathrm{x}$ & 13.0 & 0.07 & 0.05 & $10: 20$ \\
\hline
\end{tabular}


Table 3. Heat-pulse flowmeter data from the Waipahu boreholes [gal/min, gallons per minute]

\begin{tabular}{|c|c|c|c|c|c|}
\hline $\begin{array}{l}\text { Depth' } \\
\text { (feet) }\end{array}$ & Packer $^{2}$ & $\begin{array}{l}\text { Pulse time }{ }^{3} \\
\text { (seconds) }\end{array}$ & $\begin{array}{l}\text { Inverse time } \\
\text { (1/second) }\end{array}$ & $\begin{array}{l}\text { Flow }{ }^{4,5} \\
\text { (gal/min) }\end{array}$ & $\begin{array}{c}\text { Time } \\
\text { (hour:minute) }\end{array}$ \\
\hline 210 & $\mathrm{X}$ & 14.0 & -0.07 & -0.06 & $10: 24$ \\
\hline 220 & $x$ & -2.7 & -0.37 & -0.40 & $10: 34$ \\
\hline 235 & $\mathrm{O}$ & 4.5 & 0.22 & 4.60 & $10: 48$ \\
\hline 235 & $x$ & 1.0 & 1.00 & 1.90 & $10: 52$ \\
\hline 248 & $\mathrm{O}$ & 3.6 & 0.28 & 5.60 & $10: 59$ \\
\hline 270 & $\mathrm{O}$ & 2.2 & 0.47 & 9.40 & 11:06 \\
\hline 292 & $\mathrm{O}$ & 1.9 & 0.54 & 10.80 & 11:09 \\
\hline 310 & $\mathrm{O}$ & 1.2 & 0.83 & 20.00 & $11: 14$ \\
\hline 325 & $\mathrm{O}$ & 1.0 & 1.01 & 26.00 & $11: 20$ \\
\hline 345 & $\mathrm{O}$ & 0.9 & 1.11 & 31.00 & $11: 29$ \\
\hline 365 & $\mathrm{O}$ & 0.8 & 1.21 & 36.00 & $11: 35$ \\
\hline 390 & $\mathrm{O}$ & 1.0 & 0.98 & 25.00 & $11: 40$ \\
\hline 415 & $\mathrm{O}$ & 2.5 & 0.40 & 8.20 & $11: 45$ \\
\hline 433 & $X$ & 1.5 & 0.67 & 1.37 & $11: 51$ \\
\hline \multicolumn{6}{|c|}{ 3.5.3 7-20-88 Below pump $27.0 \mathrm{gal} / \mathrm{min}$ starting at 12:00 } \\
\hline 200 & $\mathrm{O}$ & $\mathrm{TF}$ & $\mathrm{TF}$ & $\mathrm{TF}$ & $12: 56$ \\
\hline 220 & $\mathrm{O}$ & TF & $\mathrm{TF}$ & TF & 13:01 \\
\hline 235 & $\mathrm{O}$ & 1.6 & 0.63 & 14.00 & $13: 04$ \\
\hline 248 & $\mathrm{O}$ & 1.6 & 0.63 & 14.00 & 13:07 \\
\hline 270 & $\mathrm{O}$ & 1.3 & 0.77 & 18.00 & $13: 11$ \\
\hline 292 & $\mathrm{O}$ & 1.3 & 0.77 & 18.00 & $13: 17$ \\
\hline 310 & $\mathrm{O}$ & 1.0 & 1.00 & 26.00 & $13: 21$ \\
\hline 325 & $\mathrm{O}$ & 1.0 & 1.00 & 26.00 & $13: 27$ \\
\hline 345 & $\mathrm{O}$ & 0.9 & 1.11 & 31.00 & $13: 33$ \\
\hline 365 & $\mathrm{O}$ & 0.9 & 1.18 & 35.00 & $13: 39$ \\
\hline 415 & $\mathrm{O}$ & 2.2 & 0.47 & 10.00 & $13: 48$ \\
\hline 433 & $\mathrm{O}$ & 12.5 & 0.08 & 1.70 & $13: 52$ \\
\hline \multicolumn{6}{|c|}{$\begin{array}{l}\text { 3.6 DATA FOR BOREHOLE OBS F } \\
3.6 .17-15-88 \text { Ambient flow data }\end{array}$} \\
\hline 215 & $\mathrm{X}$ & 16.0 & 0.06 & 0.03 & $10: 13$ \\
\hline 215 & $x$ & 5.2 & 0.19 & 0.14 & $10: 16$ \\
\hline 230 & $x$ & 1.9 & 0.52 & 0.70 & $10: 28$ \\
\hline 250 & $\mathrm{O}$ & 5.2 & 0.19 & 3.90 & $10: 34$ \\
\hline 250 & $x$ & 0.9 & 1.15 & 2.70 & $10: 39$ \\
\hline 290 & $\mathrm{O}$ & 1.3 & 0.76 & 17.60 & $10: 47$ \\
\hline 290 & $x$ & 0.7 & 1.47 & 4.20 & $10: 52$ \\
\hline 325 & $\mathrm{O}$ & 1.9 & 0.52 & 11.20 & $11: 02$ \\
\hline 350 & $\mathrm{O}$ & 2.0 & 0.50 & 10.00 & $11: 10$ \\
\hline 350 & $x$ & 0.5 & 2.00 & 6.40 & $11: 16$ \\
\hline 350 & $\mathrm{O}$ & 1.5 & 0.70 & 16.00 & $11: 21$ \\
\hline 385 & $\mathrm{O}$ & 1.3 & 0.77 & 17.90 & $11: 30$ \\
\hline 400 & 0 & 3.2 & 0.31 & 16.40 & $11: 43$ \\
\hline 413 & $\mathrm{O}$ & 9.6 & 0.10 & 2.00 & $11: 52$ \\
\hline \multicolumn{6}{|c|}{ 3.6.2 7-19-88 Ambient flow data } \\
\hline 230 & $\mathrm{O}$ & 22.0 & 0.05 & 0.90 & $14: 58$ \\
\hline 230 & $\mathrm{x}$ & 1.3 & 0.76 & 1.28 & $15: 06$ \\
\hline
\end{tabular}


Table 3. Heat-pulse flowmeter data from the Waipahu boreholes [gal/min, gallons per minute]

\begin{tabular}{|c|c|c|c|c|c|}
\hline $\begin{array}{l}\text { Depth' } \\
\text { (feet) }\end{array}$ & Packer ${ }^{2}$ & $\begin{array}{l}\text { Pulse time }{ }^{3} \\
\text { (seconds) }\end{array}$ & $\begin{array}{l}\text { Inverse time } \\
\text { (1/second) }\end{array}$ & $\begin{array}{l}\text { Flow }^{4,5} \\
(\mathrm{gal} / \mathrm{min})\end{array}$ & $\begin{array}{l}\text { Time } \\
\text { (hour:minute) }\end{array}$ \\
\hline 250 & $\mathrm{O}$ & 5.0 & 0.20 & 4.10 & $15: 16$ \\
\hline 290 & $\mathrm{O}$ & 1.4 & 0.74 & 16.90 & $15: 23$ \\
\hline 350 & $\mathrm{O}$ & 1.2 & 0.83 & 19.80 & $15: 31$ \\
\hline 375 & $\mathrm{O}$ & 1.2 & 0.86 & 20.80 & $15: 35$ \\
\hline 400 & 0 & 2.5 & 0.39 & 8.30 & $15: 39$ \\
\hline 412 & $\mathrm{O}$ & 20.0 & 0.05 & 1.00 & $15: 46$ \\
\hline 406 & 0 & 6.3 & 0.16 & 3.20 & $15: 52$ \\
\hline 409 & 0 & 7.7 & 0.13 & 2.60 & $15: 58$ \\
\hline \multicolumn{6}{|c|}{ 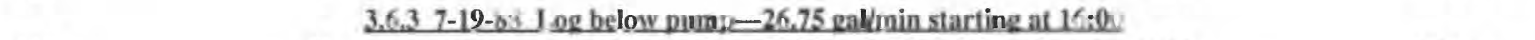 } \\
\hline 412 & $\mathrm{O}$ & 10.8 & 0.09 & 1.90 & $16: 14$ \\
\hline 400 & $\mathrm{O}$ & 2.6 & 0.39 & 8.10 & $16: 18$ \\
\hline 205 & $\mathrm{O}$ & 1.2 & 0.87 & 21.20 & $16: 48$ \\
\hline 230 & $\mathrm{O}$ & 1.2 & 0.83 & 20.00 & $16: 56$ \\
\hline 250 & $\mathrm{O}$ & 1.2 & 0.83 & 20.00 & $17: 01$ \\
\hline 270 & 0 & 0.9 & 1.18 & 34.00 & $17: 05$ \\
\hline 290 & $\mathrm{O}$ & 0.9 & 1.08 & 29.00 & $17: 10$ \\
\hline 295 & $\mathrm{O}$ & 0.9 & 1.13 & 32.00 & $17: 14$ \\
\hline 310 & $\mathrm{O}$ & 0.9 & 1.10 & 32.00 & $17: 20$ \\
\hline 325 & $\mathrm{O}$ & 0.8 & 1.23 & 38.00 & $17: 26$ \\
\hline 350 & $\mathrm{O}$ & 0.9 & 1.00 & 26.00 & $17: 31$ \\
\hline 362 & $\mathrm{O}$ & 0.9 & 1.12 & 32.00 & $17: 46$ \\
\hline 375 & $\mathrm{O}$ & 0.9 & 1.09 & 30.00 & $17: 54$ \\
\hline 390 & $\mathrm{O}$ & 2.1 & 0.47 & 10.10 & $18: 02$ \\
\hline 409 & $\mathrm{O}$ & 4.0 & 0.25 & 5.10 & $18: 11$ \\
\hline \multicolumn{6}{|c|}{ 3.6.4 3-15-89 Ambient flow data } \\
\hline 220 & $x$ & -2.4 & -0.41 & -0.45 & $11: 27$ \\
\hline 246 & $\mathrm{x}$ & 4.0 & 0.25 & 0.22 & $11: 36$ \\
\hline 280 & $\mathrm{X}$ & 2.5 & 0.40 & 0.45 & $11: 54$ \\
\hline 310 & $\mathrm{O}$ & 3.1 & 0.33 & 6.90 & $12: 28$ \\
\hline 335 & $\mathrm{O}$ & 7.8 & 0.13 & 3.30 & $12: 32$ \\
\hline 335 & $x$ & 1.1 & 0.88 & 1.70 & $12: 38$ \\
\hline 355 & $\mathrm{O}$ & 14.1 & 0.07 & 1.60 & $12: 47$ \\
\hline 374 & $x$ & 1.3 & 0.76 & 1.30 & $12: 58$ \\
\hline 394 & $\mathrm{X}$ & 1.0 & 0.99 & 2.10 & $13: 05$ \\
\hline 408 & $x$ & 1.5 & 0.67 & 1.05 & $13: 11$ \\
\hline \multicolumn{6}{|c|}{$\begin{array}{l}\text { 3.7 DATA FOR BOREHOLE OBS H } \\
3.7 .17-26-88 \text { Ambient flow data }\end{array}$} \\
\hline 230 & $x$ & 3.7 & 0.27 & 0.23 & $14: 48$ \\
\hline 230 & $\mathrm{O}$ & 16.0 & 0.06 & 1.30 & $14: 54$ \\
\hline 250 & $x$ & 18.5 & 0.56 & 0.77 & $15: 03$ \\
\hline 250 & $\mathrm{O}$ & 18.5 & 0.05 & 1.20 & $15: 08$ \\
\hline 270 & $x$ & -1.2 & -0.93 & 1.54 & $15: 17$ \\
\hline 270 & $\mathrm{O}$ & -4.5 & -0.22 & -5.90 & $15: 22$ \\
\hline 290 & $x$ & -2.0 & 0.50 & 0.61 & $15: 28$ \\
\hline 290 & $\mathrm{O}$ & 20.0 & 0.07 & 1.40 & $15: 32$ \\
\hline 310 & $x$ & 3.0 & 0.33 & 0.31 & $15: 44$ \\
\hline
\end{tabular}


Table 3. Heat-pulse flowmeter data from the Waipahu boreholes [gal/min, gallons per minute]

\begin{tabular}{|c|c|c|c|c|c|}
\hline $\begin{array}{l}\text { Depth } \\
\text { (feet) }\end{array}$ & Packer $^{2}$ & $\begin{array}{l}\text { Pulse time } \\
\text { (seconds) }\end{array}$ & $\begin{array}{l}\text { Inverse time } \\
\text { (1/second) }\end{array}$ & $\begin{array}{l}\text { Flow } 4,5 \\
\text { (gal/min) }\end{array}$ & $\begin{array}{l}\text { Time } \\
\text { (hour:minute) }\end{array}$ \\
\hline 330 & $x$ & 1.4 & 0.71 & 1.12 & $15: 53$ \\
\hline 330 & $\mathrm{O}$ & 8.0 & 0.13 & 2.60 & $15: 58$ \\
\hline 350 & $\mathrm{O}$ & 5.5 & 0.18 & 3.70 & $16: 07$ \\
\hline 376 & $\mathrm{O}$ & 11.1 & 0.09 & 1.80 & $16: 15$ \\
\hline 376 & $X$ & 1.5 & 0.68 & 1.00 & $16: 19$ \\
\hline 390 & $\mathrm{X}$ & 1.9 & 0.52 & 0.65 & $16: 24$ \\
\hline 390 & 0 & 9.0 & 0.11 & 2.40 & $16: 32$ \\
\hline 410 & $X$ & 3.6 & 0.28 & 0.25 & $16: 42$ \\
\hline 436 & $x$ & -9.0 & -0.11 & -0.09 & $16: 53$ \\
\hline \multicolumn{6}{|c|}{$\begin{array}{l}\text { 3.8 DATA FOR BOREHOLE DEEP OBS } \\
3.8 .1 \text { 6-26-87 Ambient flow data }\end{array}$} \\
\hline 405 & $x$ & -5.1 & -0.20 & -0.16 & $14: 10$ \\
\hline 398 & $\mathrm{O}$ & 8.0 & 0.12 & 2.50 & $14: 20$ \\
\hline 562 & $\mathrm{O}$ & -7.1 & -0.14 & -4.80 & $14: 40$ \\
\hline 562 & $\mathrm{X}$ & -1.4 & -0.71 & -1.10 & $14: 44$ \\
\hline 644 & $\mathrm{O}$ & -14.7 & -0.07 & -1.80 & $14: 50$ \\
\hline 735 & $\mathrm{O}$ & 4.5 & 0.22 & 4.50 & $15: 01$ \\
\hline 800 & $\mathrm{O}$ & 5.1 & 0.20 & 4.00 & $15: 06$ \\
\hline 994 & $\mathrm{O}$ & 28.0 & 0.04 & 0.70 & $15: 17$ \\
\hline 1160 & $\mathrm{O}$ & 23.0 & 0.04 & 0.90 & $15: 32$ \\
\hline 1160 & $\mathrm{X}$ & 6.8 & 0.15 & 0.11 & $15: 39$ \\
\hline 1226 & $\mathrm{O}$ & 27.0 & 0.04 & 0.70 & $15: 46$ \\
\hline 1385 & $\mathrm{O}$ & 17.0 & 0.06 & 1.20 & $16: 20$ \\
\hline 1385 & $x$ & 5.3 & 0.19 & 0.16 & $16: 25$ \\
\hline 1547 & $\mathrm{O}$ & 17.0 & 0.06 & 1.20 & $16: 40$ \\
\hline 1547 & $X$ & 5.7 & 0.17 & 0.14 & $16: 52$ \\
\hline 675 & $\mathrm{O}$ & -32.0 & -0.03 & -0.80 & $17: 23$ \\
\hline 512 & $\mathrm{O}$ & -60.0 & -0.02 & -0.40 & $17: 38$ \\
\hline 440 & $\mathrm{O}$ & 60.0 & 0.02 & 0.30 & $17: 51$ \\
\hline \multicolumn{6}{|c|}{ 3.8.2 7-27-88 Ambient flow data } \\
\hline 410 & $\mathrm{x}$ & -0.9 & -1.11 & -2.70 & $14: 50$ \\
\hline 410 & $\mathrm{O}$ & -11.0 & -0.09 & -2.60 & $14: 56$ \\
\hline 458 & $\mathrm{O}$ & 9.9 & 0.10 & 2.00 & $15: 02$ \\
\hline 458 & $\mathrm{X}$ & -1.3 & -0.77 & -1.23 & $15: 10$ \\
\hline 525 & $x$ & TF & $\mathrm{TF}$ & $\mathrm{TF}$ & $15: 26$ \\
\hline 525 & $\mathrm{O}$ & 3.8 & 0.26 & 5.30 & $15: 33$ \\
\hline 562 & $X$ & TF & $\mathrm{TF}$ & $\mathrm{TF}$ & $15: 48$ \\
\hline 562 & $\mathrm{O}$ & -3.5 & -0.29 & -6.90 & $15: 56$ \\
\hline 1167 & $X$ & 5.1 & 0.20 & 0.17 & $16: 30$ \\
\hline 1135 & $x$ & 10.0 & 0.10 & 0.08 & $16: 42$ \\
\hline \multicolumn{6}{|c|}{$3.8 .3 \quad 7-28-88$ Ambient flow data } \\
\hline 458 & $\mathrm{O}$ & 15.0 & 0.07 & 1.40 & $10: 08$ \\
\hline 458 & $X$ & -1.0 & -1.00 & -1.80 & $10: 17$ \\
\hline 1167 & $\mathrm{X}$ & 1.0 & 1.00 & 1.90 & $10: 44$ \\
\hline 1167 & $\mathrm{O}$ & 3.0 & 0.33 & 7.00 & $10: 53$ \\
\hline 1608 & $\mathrm{O}$ & 4.6 & 0.22 & 4.40 & $11: 23$ \\
\hline
\end{tabular}


Table 3. Heat-pulse flowmeter data from the Waipahu boreholes [gal/min, gallons per minute]

\begin{tabular}{|c|c|c|c|c|c|}
\hline $\begin{array}{l}\text { Depth } 1 \\
\text { (feet) }\end{array}$ & Packer $^{2}$ & $\begin{array}{l}\text { Pulse time } \\
\text { (seconds) }\end{array}$ & $\begin{array}{l}\text { Inverse time } \\
\text { (1/second) }\end{array}$ & $\begin{array}{l}\text { Flow } 4,5 \\
\text { (gal/min) }\end{array}$ & $\begin{array}{c}\text { Time } \\
\text { (hour:minute) }\end{array}$ \\
\hline 1608 & $\mathrm{O}$ & 12.1 & 0.08 & 1.70 & $11: 30$ \\
\hline 1608 & $\mathrm{X}$ & 3.9 & 0.26 & 0.26 & $11: 42$ \\
\hline 1547 & $\mathrm{O}$ & 12.4 & 0.08 & 1.60 & $11: 56$ \\
\hline 1547 & $\mathrm{X}$ & 5.0 & 0.20 & 0.20 & $12: 40$ \\
\hline 1436 & $x$ & 2.3 & 0.43 & 0.47 & $13: 16$ \\
\hline 1220 & $\mathrm{O}$ & 5.5 & 0.18 & 3.70 & $15: 40$ \\
\hline 1167 & $\mathrm{O}$ & 23.0 & 0.04 & 0.80 & $16: 25$ \\
\hline 562 & $\mathrm{O}$ & -1.4 & -0.71 & -14.50 & $16: 44$ \\
\hline \multicolumn{6}{|c|}{ 3.8.4 3-8-89 Ambient flow data } \\
\hline 411 & $X$ & -1.3 & -0.79 & -1.51 & $15: 58$ \\
\hline 410 & $\mathrm{x}$ & -1.7 & -0.58 & -0.83 & $16: 11$ \\
\hline 458 & $\mathrm{X}$ & -1.5 & -0.64 & -1.00 & $16: 24$ \\
\hline 525 & $x$ & -0.8 & -1.31 & -4.40 & $16: 37$ \\
\hline \multicolumn{6}{|c|}{3.8 .5 3-9-89 Ambient flow data } \\
\hline 410 & $X$ & -1.2 & -0.84 & -1.72 & $09: 40$ \\
\hline 458 & $\mathrm{X}$ & -1.4 & -0.73 & -1.27 & $09: 56$ \\
\hline 525 & $\mathrm{O}$ & 7.6 & 0.13 & 2.70 & $10: 08$ \\
\hline 525 & $\mathrm{X}$ & 1.4 & 0.07 & 1.14 & $10: 22$ \\
\hline 562 & $x$ & -0.7 & -1.35 & -4.94 & $10: 31$ \\
\hline 562 & $\mathrm{O}$ & -1.7 & -0.62 & -12.60 & $10: 38$ \\
\hline 688 & 0 & -4.7 & -0.21 & -4.30 & $10: 50$ \\
\hline 688 & $x$ & -1.0 & -1.00 & -2.51 & $10: 58$ \\
\hline 688 & $\mathrm{O}$ & -7.0 & -0.14 & -2.90 & 11:06 \\
\hline 730 & $x$ & 2.1 & 0.47 & 0.58 & $11: 16$ \\
\hline 730 & $\mathrm{O}$ & 5.8 & 0.17 & 3.50 & $11: 26$ \\
\hline 905 & $\mathrm{X}$ & -1.2 & -0.82 & -1.61 & $11: 55$ \\
\hline 958 & $x$ & -1.0 & -1.00 & -2.56 & $12: 14$ \\
\hline 1095 & $\mathrm{O}$ & 4.3 & 0.23 & 4.80 & $12: 27$ \\
\hline 1095 & $\mathrm{x}$ & -2.3 & -0.43 & -0.48 & $13: 51$ \\
\hline 1135 & $\mathrm{x}$ & 1.6 & 0.64 & 0.96 & $14: 07$ \\
\hline 1167 & $x$ & 1.9 & 0.53 & 0.80 & $14: 20$ \\
\hline \multicolumn{6}{|c|}{ 3.8.6 3-10-89 Ambient flow data } \\
\hline 458 & $x$ & -1.2 & -0.85 & -1.89 & $16: 05$ \\
\hline 1135 & $\mathrm{X}$ & 0.9 & 1.08 & 2.38 & $16: 38$ \\
\hline 1167 & $X$ & 1.0 & 1.03 & 2.21 & $16: 52$ \\
\hline 1190 & $\mathrm{X}$ & 1.3 & 0.77 & 1.33 & $17: 06$ \\
\hline 1190 & $\mathrm{O}$ & 8.9 & 0.11 & 2.20 & $17: 18$ \\
\hline 1220 & $\mathrm{X}$ & 5.3 & 0.19 & 0.15 & $17: 28$ \\
\hline 1220 & $\mathrm{O}$ & 8.2 & 0.12 & 2.30 & $17: 36$ \\
\hline 1220 & $\mathrm{O}$ & 13.4 & 0.08 & 1.70 & $17: 44$ \\
\hline 1220 & $x$ & 9.3 & 0.11 & 0.07 & $17: 57$ \\
\hline \multicolumn{6}{|c|}{ 3.8.7 3-13-89 Ambient flow data } \\
\hline 458 & $x$ & -1.2 & -0.83 & -1.55 & $09: 44$ \\
\hline 996 & $X$ & -0.9 & -1.11 & -2.60 & $10: 09$ \\
\hline 1135 & $X$ & 1.4 & 0.71 & 1.66 & $10: 30$ \\
\hline 1312 & $\mathrm{X}$ & 12.0 & 0.08 & 0.06 & $11: 02$ \\
\hline
\end{tabular}


Table 3. Heat-pulse flowmeter data from the Waipahu boreholes [gal/min, gallons per minute]

\begin{tabular}{|c|c|c|c|c|c|}
\hline $\begin{array}{l}\text { Depth }^{1} \\
\text { (feet) }\end{array}$ & Packer ${ }^{2}$ & $\begin{array}{l}\text { Pulse time }{ }^{3} \\
\text { (seconds) }\end{array}$ & $\begin{array}{l}\text { Inverse time } \\
\text { (1/second) }\end{array}$ & $\begin{array}{l}\text { Flow }^{4,5} \\
\text { (gal/min) }\end{array}$ & $\begin{array}{c}\text { Time } \\
\text { (hour:minute) }\end{array}$ \\
\hline \multicolumn{6}{|c|}{ 3.8.8 3-14-88 Ambient flow data } \\
\hline 458 & $x$ & -1.0 & -1.00 & -2.50 & $14: 36$ \\
\hline 1430 & $\mathrm{X}$ & 3.2 & 0.31 & 0.31 & $15: 28$ \\
\hline 1547 & $\mathrm{X}$ & 3.4 & 0.30 & 0.10 & $16: 02$ \\
\hline 1547 & $\mathrm{X}$ & 7.0 & 0.14 & 0.06 & $16: 06$ \\
\hline 1547 & $\mathrm{X}$ & 14.8 & 0.07 & 0.04 & $16: 08$ \\
\hline 1500 & $\mathrm{X}$ & -30.0 & -0.03 & -0.02 & $16: 30$ \\
\hline 1386 & $\mathrm{X}$ & 12.1 & 0.08 & 0.05 & $16: 53$ \\
\hline 1348 & $x$ & 30.0 & 0.03 & 0.01 & $17: 10$ \\
\hline 1312 & $\mathrm{X}$ & -30.0 & -0.03 & -0.01 & $17: 32$ \\
\hline 1167 & $\mathrm{X}$ & -6.9 & -0.15 & -0.10 & $17: 58$ \\
\hline 996 & $x$ & -0.8 & -1.23 & -4.00 & $18: 20$ \\
\hline
\end{tabular}

${ }^{1}$ Depth in feet from top of surface casing; values are assumed to be within $\pm 1.0 \mathrm{ft}$.

${ }^{2} \mathrm{X}$ denotes measurements made with inflated packer; $\mathrm{O}$ denotes measurements made with uninflated packer.

${ }^{3}$ Each listed pulse time is an average of three or more separate measurements made at the given depth station.

${ }^{4}$ Minus sign indicates downflow; otherwise upflow is indicated.

${ }^{5}$ Flow calibrations used for appropriate tool configuration and approximate borehole diameter for flowmeter with packer deflated; with packer inflated, flows are $\pm 0.05 \mathrm{gal} / \mathrm{min}$; with packer deflated, llows are \pm 50 percent; flows less than $1.0 \mathrm{gal} / \mathrm{min}$ measured with uninflated packer may not be meaningfil and sometimes represent thermally driven convection flows rather than net borehole flows.

${ }^{6} \mathrm{NF}$ indicates no consistent peaks and flowmeter response otherwise indicative of no flow or flow below the $0.01 \mathrm{gal} / \mathrm{min}$ resolution of the HPFM.

${ }^{7}$ TF response indicates flow probably too fast to measure with the HPFM.

${ }^{8} \mathrm{~N}$ denotes noisy and unstable signal measured with uninflated packer, often indicating measurements made near an inflow point; this turbulent effect persists for at least five borehole diameters above or below entry point. 
The combination of fixed-depth mode and trolling mode measurements were effective in defining both the magnitude and distribution of flows in the Waipahu Mahoe boreholes. For example, the trolling-mode data obtained for borehole Obs A effectively indicate depths where flow was entering or exiting the boreholes (fig. $14 \mathrm{~A}$ ), but do not give especially accurate values because of the relatively large uncertainties associated with measurements made with the downhole packer deflated and caused by the turbulence and flow bias introduced by the trolling. The steps in the flow profile associated with inflow and outflow are indicated by large shifts in the relatively noisy plot of trolling-mode data. The trolling-mode data can be corrected for the trolling bias by superimposing the stationary-mode measurements on the same plot and shifting the trolling-mode data plot to fit those stationary flow measurement points where borehole flow is much more accurately determined and there is no trolling bias (fig. 14B). This shifted curve can then be used to identify depths where flow is entering or exiting from the borehole under ambient flow conditions (fig. 14C). Note that these inflows and outflows are inferred from data obtained in July 1988 and are different from inflows and outflows given for June 1987 in figure 5. In comparing the inflow and outflow data given in figure $14 \mathrm{C}$ with data in figure 5 , the depths where inflow or outflow occur are comparable, but the amount of inflow or outflow in each figure is a function of the background hydraulic conditions at the time of logging.

The combination of trolling-mode and fixed-mode data sets for the Waipahu Mahoe boreholes can be used to estimate the amounts of flow and the entry and exit depths for inflows and outflows to the boreholes induced by the ambient hydraulic-head conditions at the time of logging. These inflows and outflows are indicated on figures 5-11, and were obtained under ambient conditions on the date indicated in the figures. The data show that almost all of the changes in slope on the temperature and fluid resistivity logs for the seven Waipahu Mahoe boreholes correspond to inflow and outflow. All of these inflows and outflows also correspond with major openings between basalt flows indicated on the caliper log. It is apparent that some of the large openings (where the size of the opening is indicated by the deflection of the caliper arms and the depth interval over which those deflections occur) were associated with large inflows, and some were not, at the time of logging. This is attributed to the fact that the local size of an opening is not the only factor controlling the horizontal transmissivity of the aquifer. Transmissivity depends upon the lateral extent of borehole wall openings and the connections between openings along the flow path between boreholes. The inflows and outflows in figures 5-11 also depended upon the hydraulic-head differences driving the flows at the time of logging.

The pattern of hydraulic connections in the aquifer at the study site can be investigated by comparing the flows induced in each of the seven Waipahu Mahoe Observation boreholes under ambient hydraulic-head conditions, and assuming that these flows are primarily induced by the pumping from the Waipahu Mahoe well field located near the observation boreholes. The lateral continuity of the openings indicated in each borehole by the caliper logs (fig. 5-11) can be investigated by searching for lateral correlations between lava flows encountered by boreholes. If the caliper logs in figures 5-11 are aligned using the depth where a dense, geochemically similar lava flow is encountered by the boreholes and if the water level in the boreholes is assumed to lie near sea level, the correlation of the logs shows that some but not all of the openings can be correlated from borehole to borehole (fig. 21).

The fixed-depth flow measuring mode HPFM data in table 3 can also be used to identify changes in the pattern of flow over time because measurements were made in different years in some of the Waipahu Mahoe observation boreholes, and because small changes in flow are more readily recognized in the accurate fixed-mode HPFM data. The comparison of fixed-mode data points for borehole Obs A indicates that there were some minor changes in the pattern of flow in the aquifer (fig. 14D). These changes are attributed to changes in the pumping rates from the Mahoe production well field and other changes in pumping in the vicinity of the study site that occurred over the measurement period. 


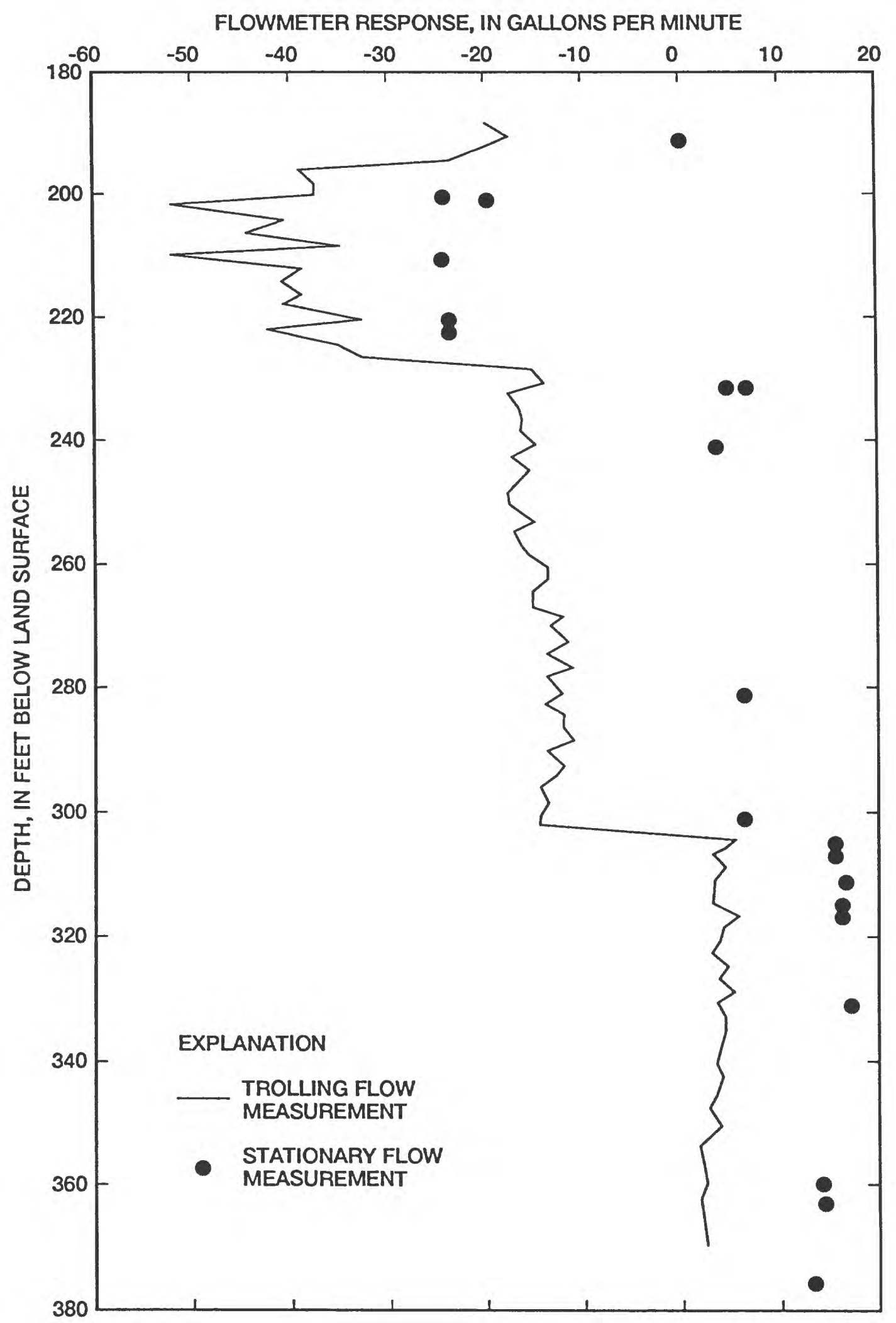

Figure 14. Heat-pulse flowmeter data obtained in borehole Obs A: (A) Flowmeter data obtained while trolling uphole with the packer deflated. 


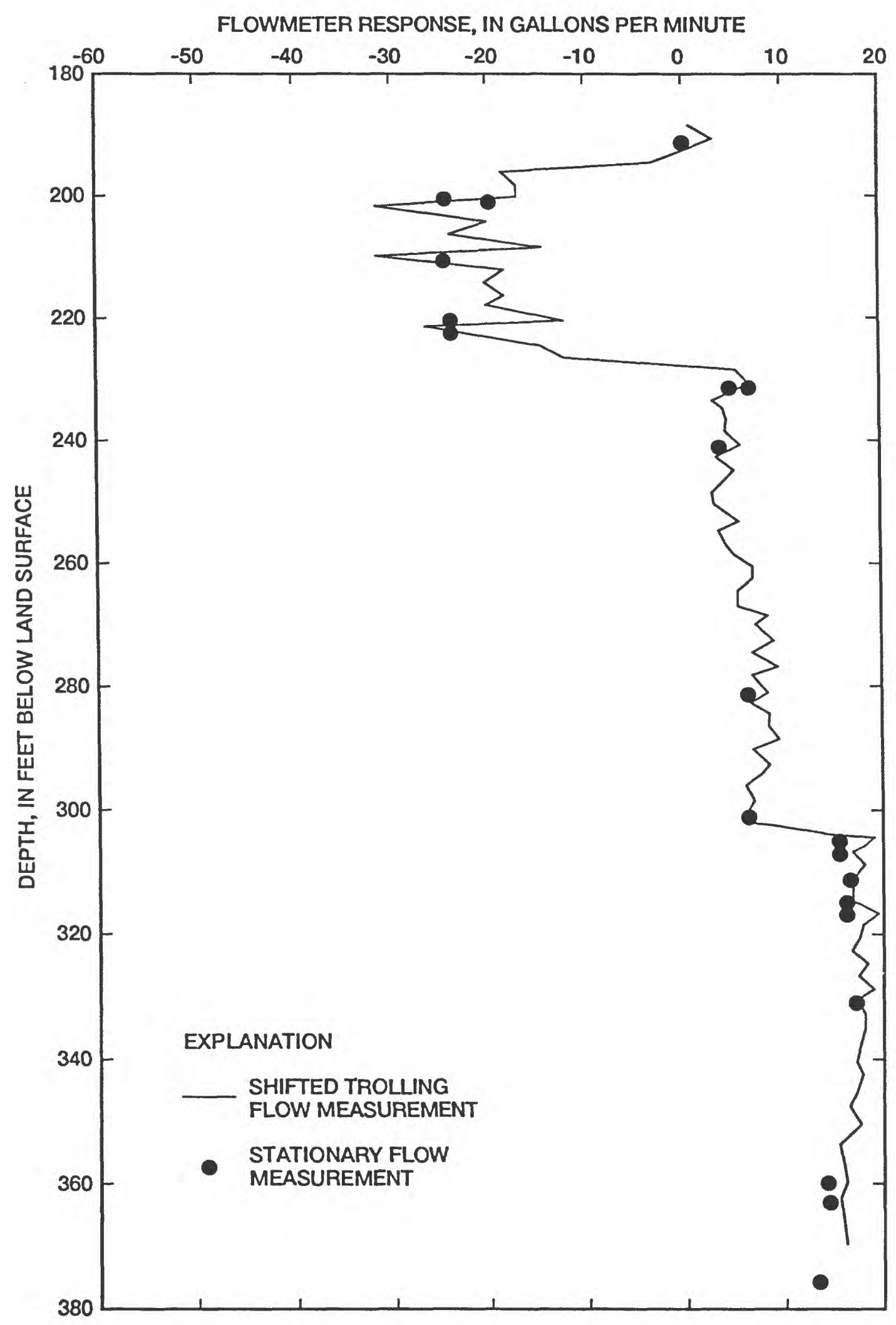

Figure 14. (B) Data after correction to account for bias of trolling speed. 


\section{FIGURES}

1. Schematic diagram showing a fracture intersecting a cylindrical borehole, and the interpretation of fracture strike and dip from the acoustic televiewer borehole image log..................................................................................

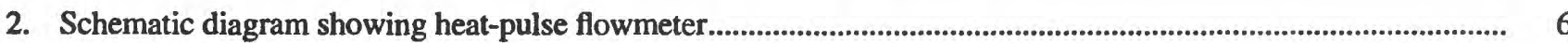

3. Graph showing typical calibration chart for a heat-pulse flowmeter with an inflated packer in place in a 6-inch diameter column

4. Map showing location of the Waipahu Mahoe Observation boreholes (Obs A through Obs $\mathrm{H}$ ), the Mahoe Pumping Station, and the Waipio Deep Observation borehole at the Waipahu, Oahu study site

5. Caliper, single-point resistance, and temperature logs for borehole Obs A compared to the interpretation of flowmeter logs showing the depths where water is entering and exiting under ambient hydraulic-head conditions; flow data obtained on 6-23-87

6. Caliper, single-point resistance, and temperature logs for borehole Obs B compared to the interpretation of the flowmeter logs showing the depths where water is entering and exiting under ambient hydraulic-head conditions; flow data obtained on 6-30-87

7. Caliper, single-point resistance, and temperature logs for borehole Obs $\mathrm{C}$ compared to the interpretation of the flowmeter logs showing the depths where water is entering and exiting under ambient hydraulic-head conditions; flow data obtained on 7-22-88

8. Caliper, single-point resistance, and temperature logs for borehole Obs D compared to the interpretation of the flowmeter logs showing the depths where water is entering and exiting under ambient hydraulic-head conditions; flow data obtained on 7-13-88.

9. Caliper, single-point resistance and temperature logs for borehole Obs E compared to the interpretation of the flowmeter logs showing the depths where water is entering and exiting under ambient hydraulic-head conditions; flow data obtained on 7-20-88

10. Caliper, single-point resistance and temperature logs for borehole Obs F compared to the interpretation of the flowmeter logs showing the depths where water is entering and exiting under ambient hydraulic-head conditions; flow data obtained on 7-15-88

11. Caliper, single-point resistance, and temperature logs for borehole Obs $\mathrm{H}$ compared to the interpretation of the flowmeter logs showing the depths where water is entering and exiting under ambient hydraulic-head 


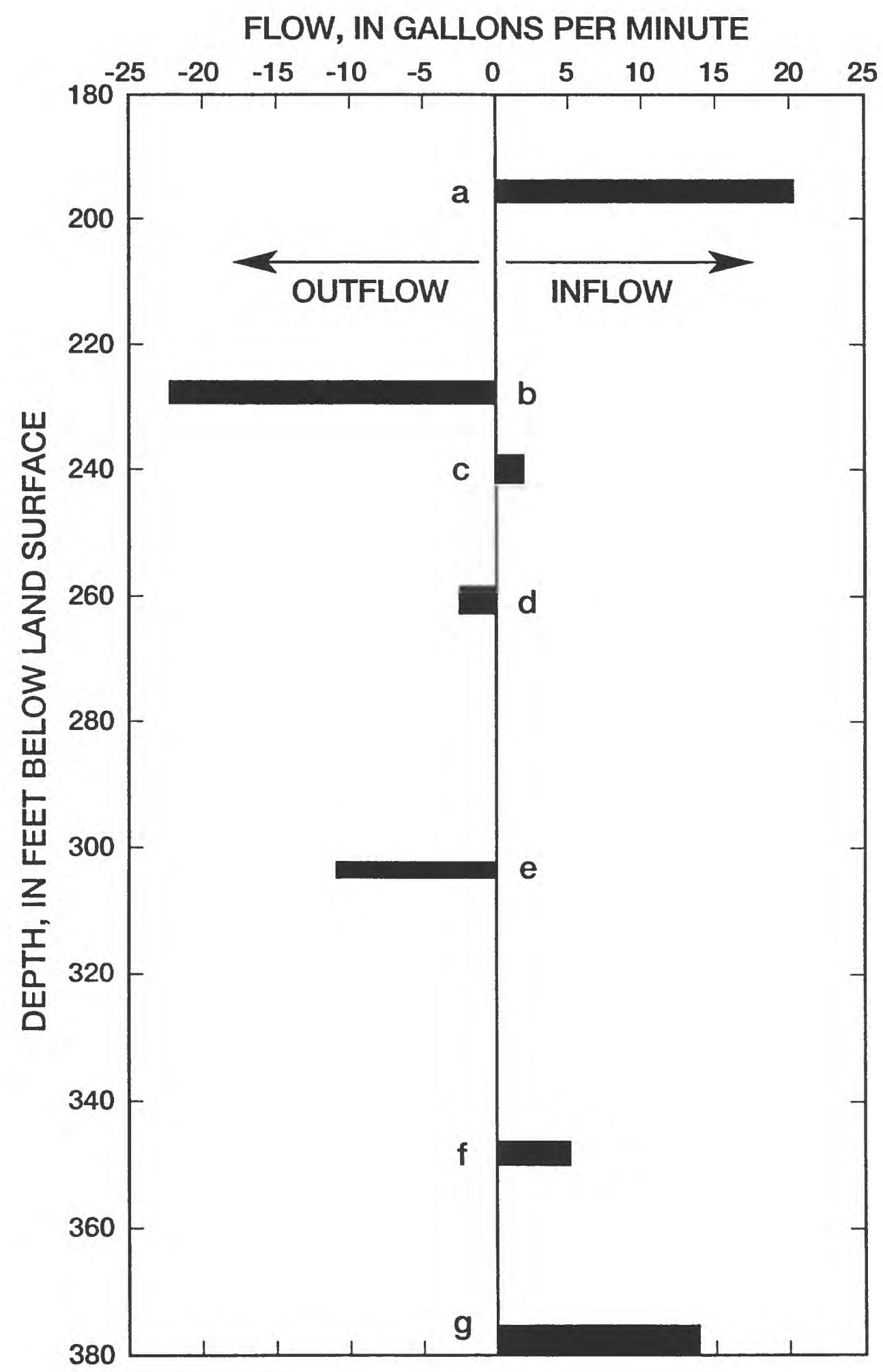

Figure 14. (C) Interpretation of inflow and outflow based on interpretation of the flowmeter data. 

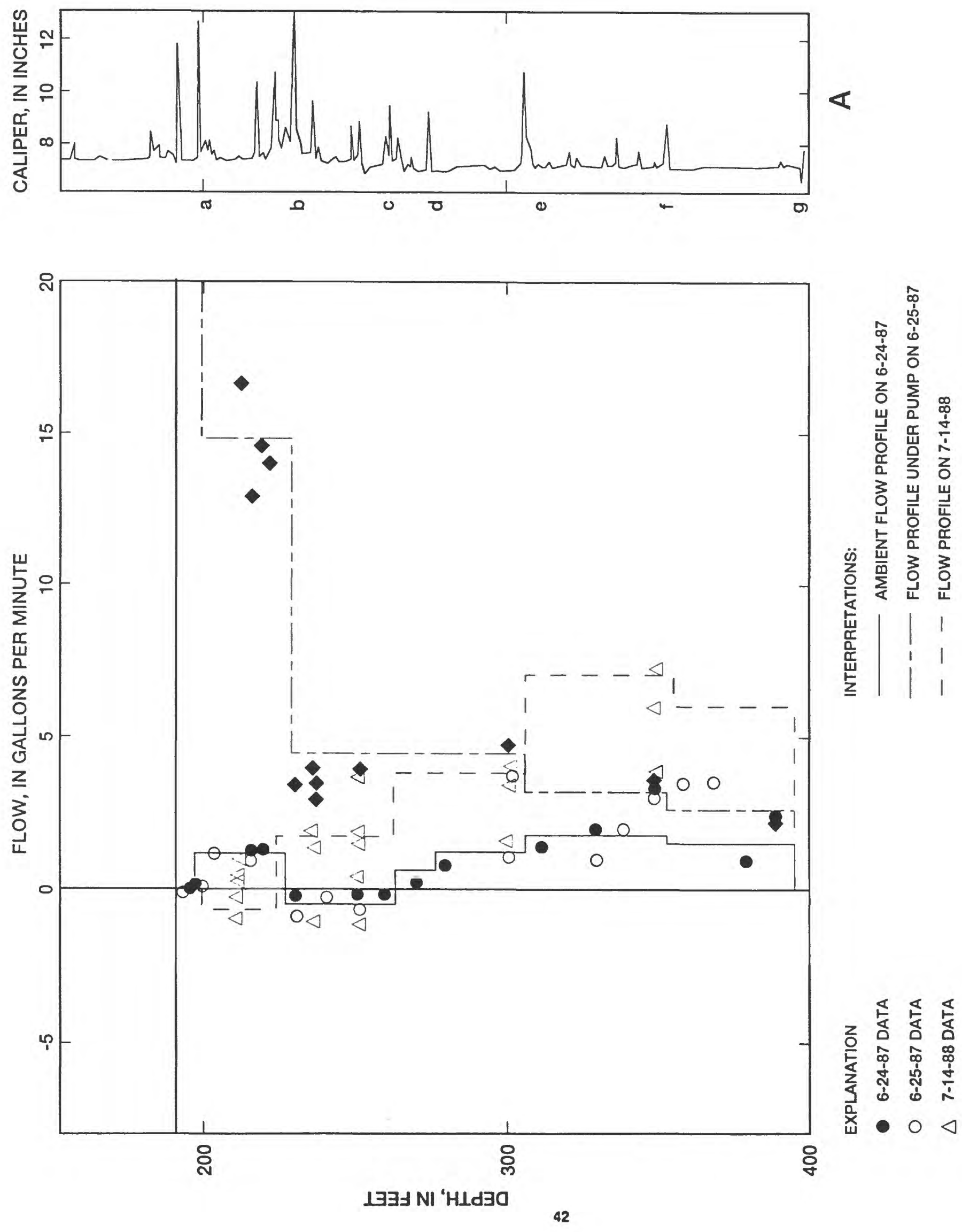

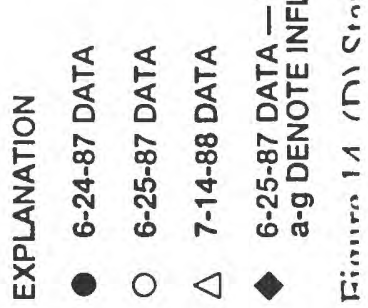




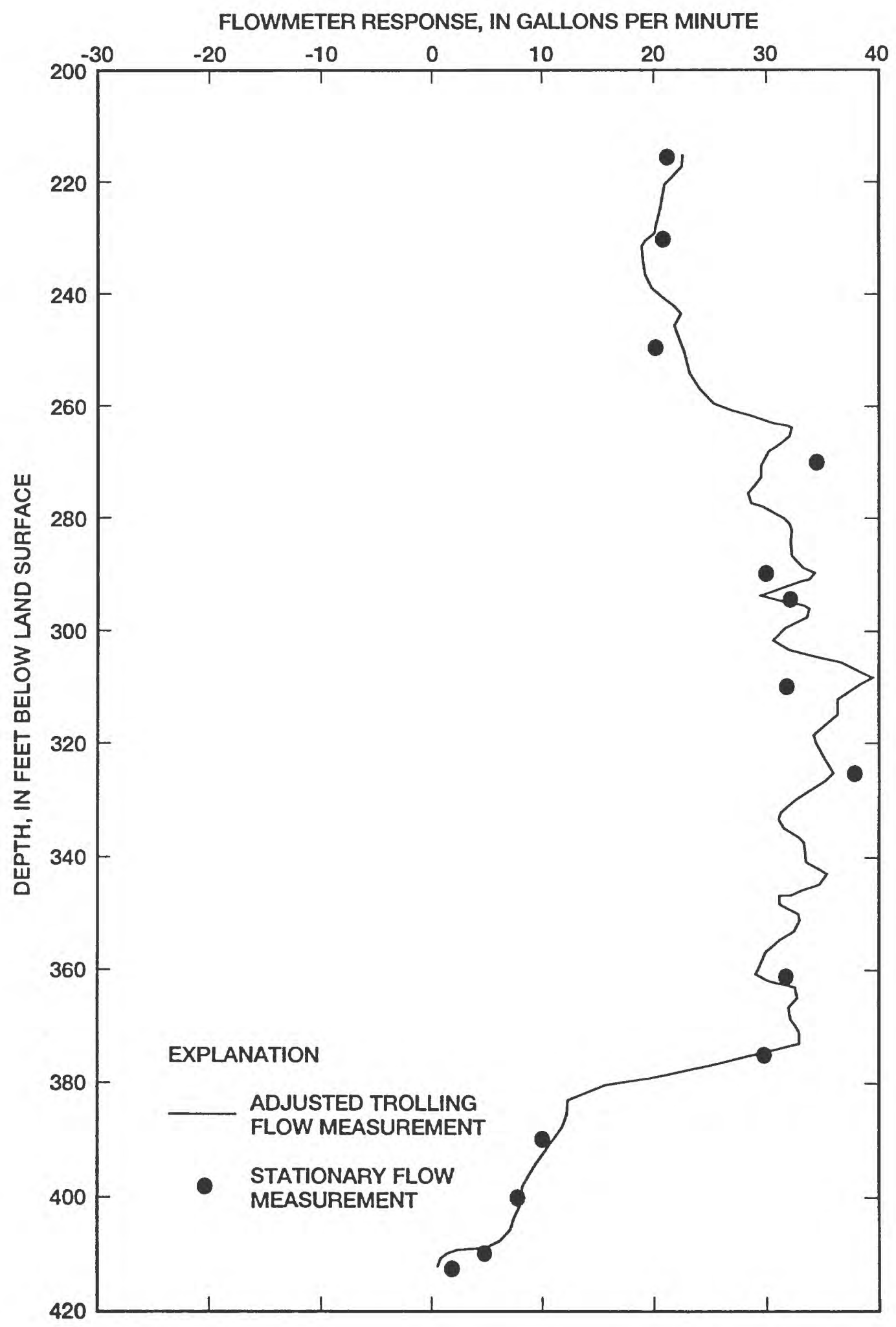

Figure 15. Heat-pulse flowmeter data obtained in borehole Obs B. 


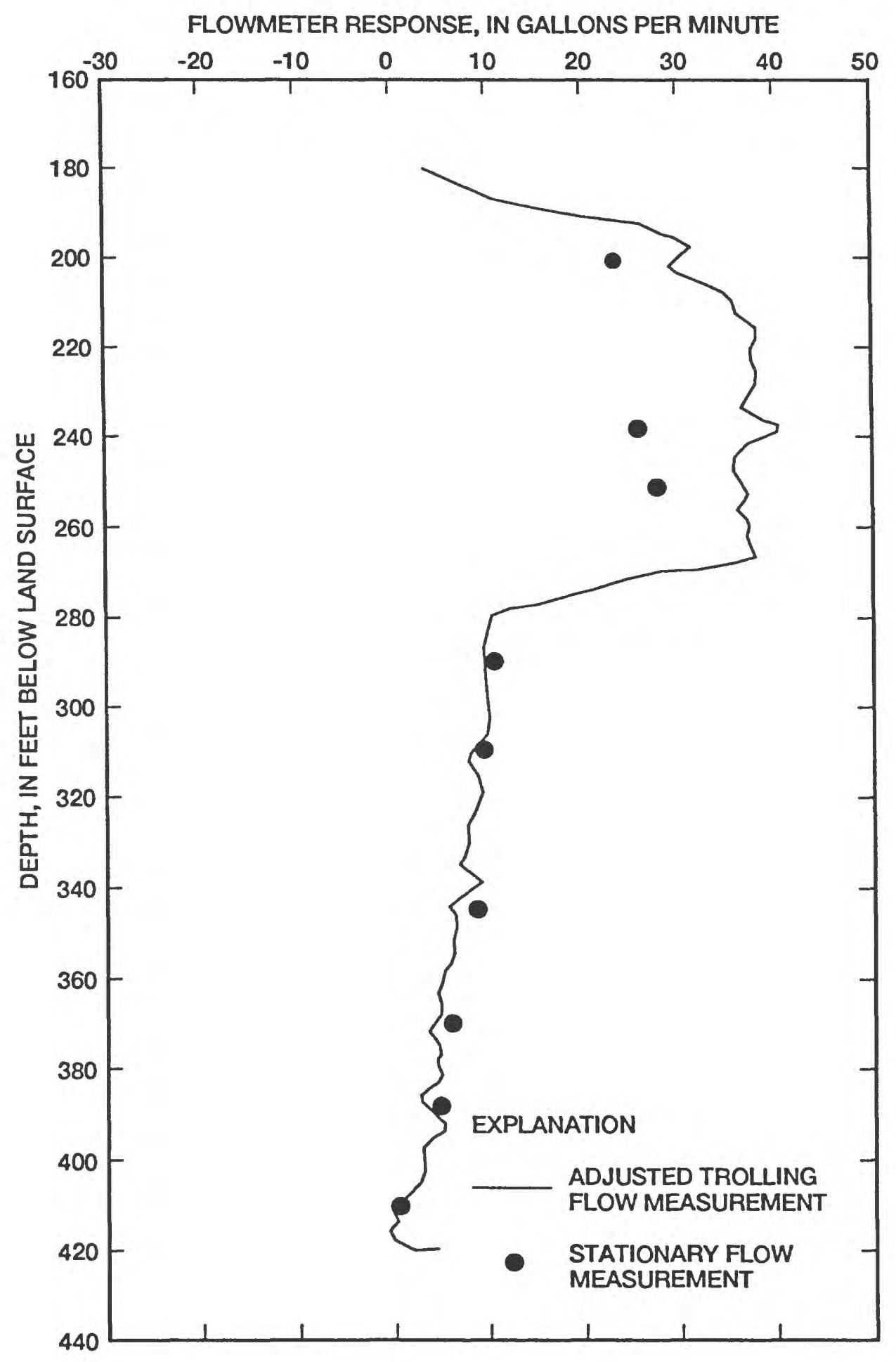

Figure 16. Heat-pulse flowmeter data obtained in borehole Obs C. 


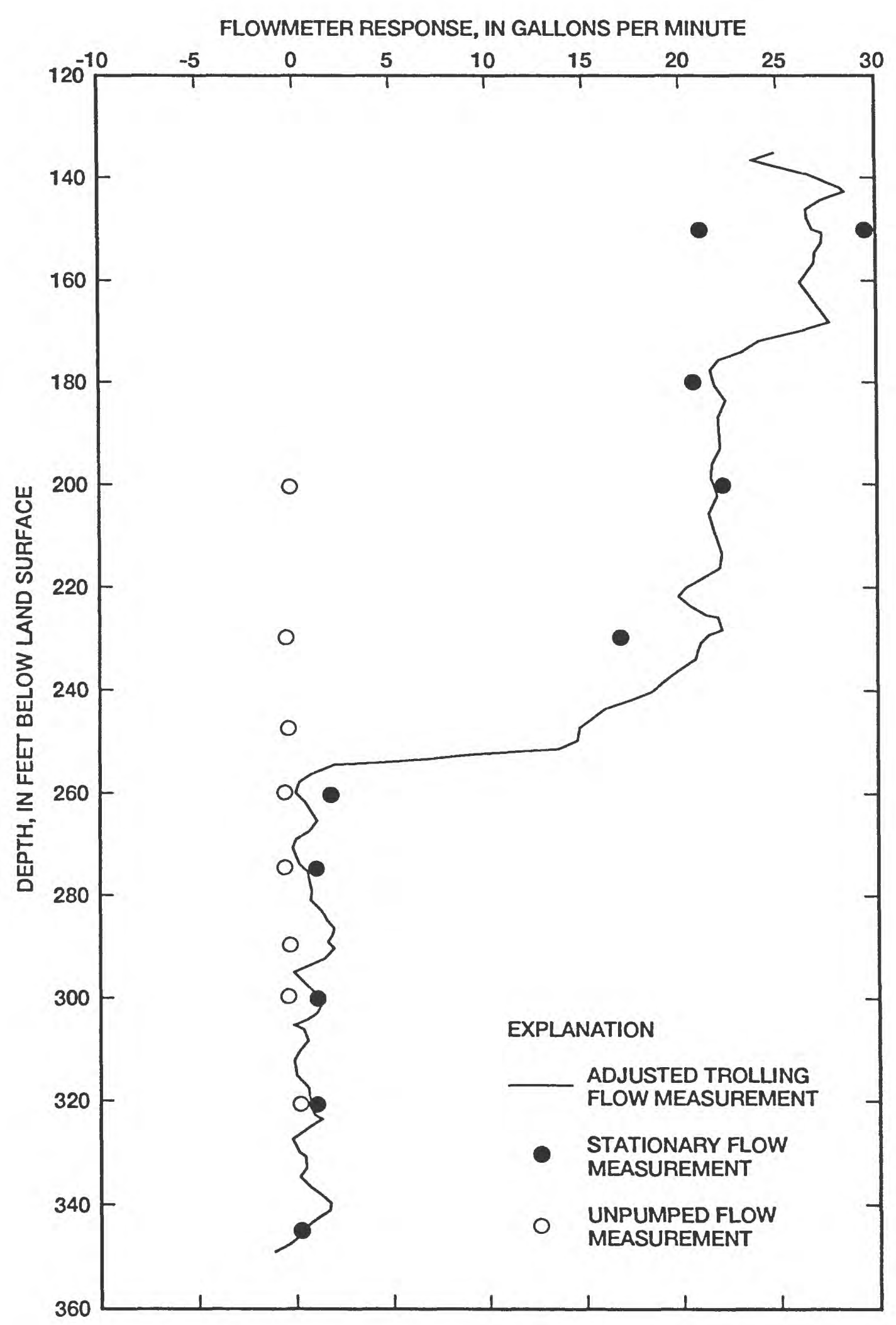

Figure 17. Heat-pulse flowmeter data obtained in borehole Obs D. 


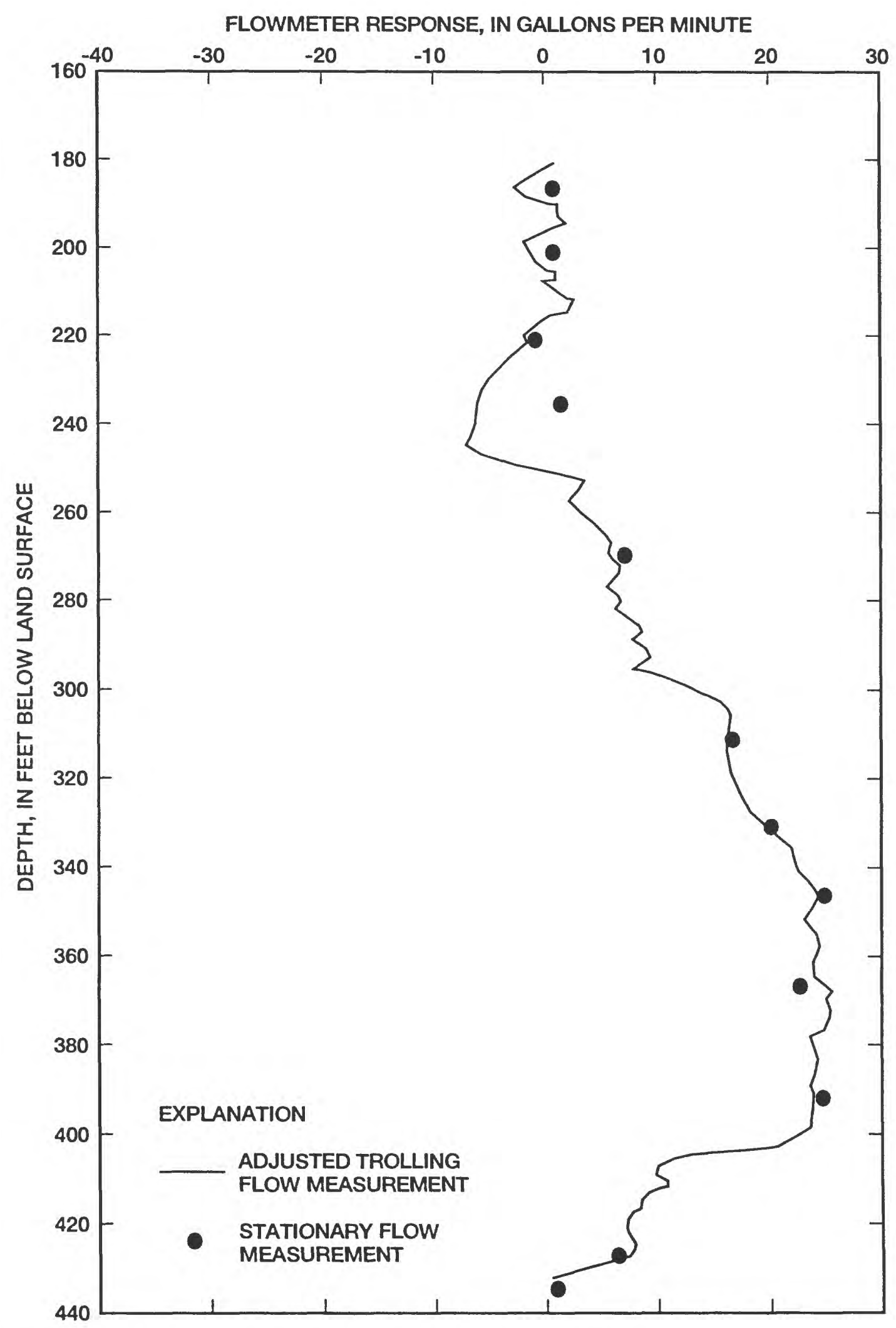

Figure 18. Heat-pulse flowmeter data obtained in borehole Obs E. 


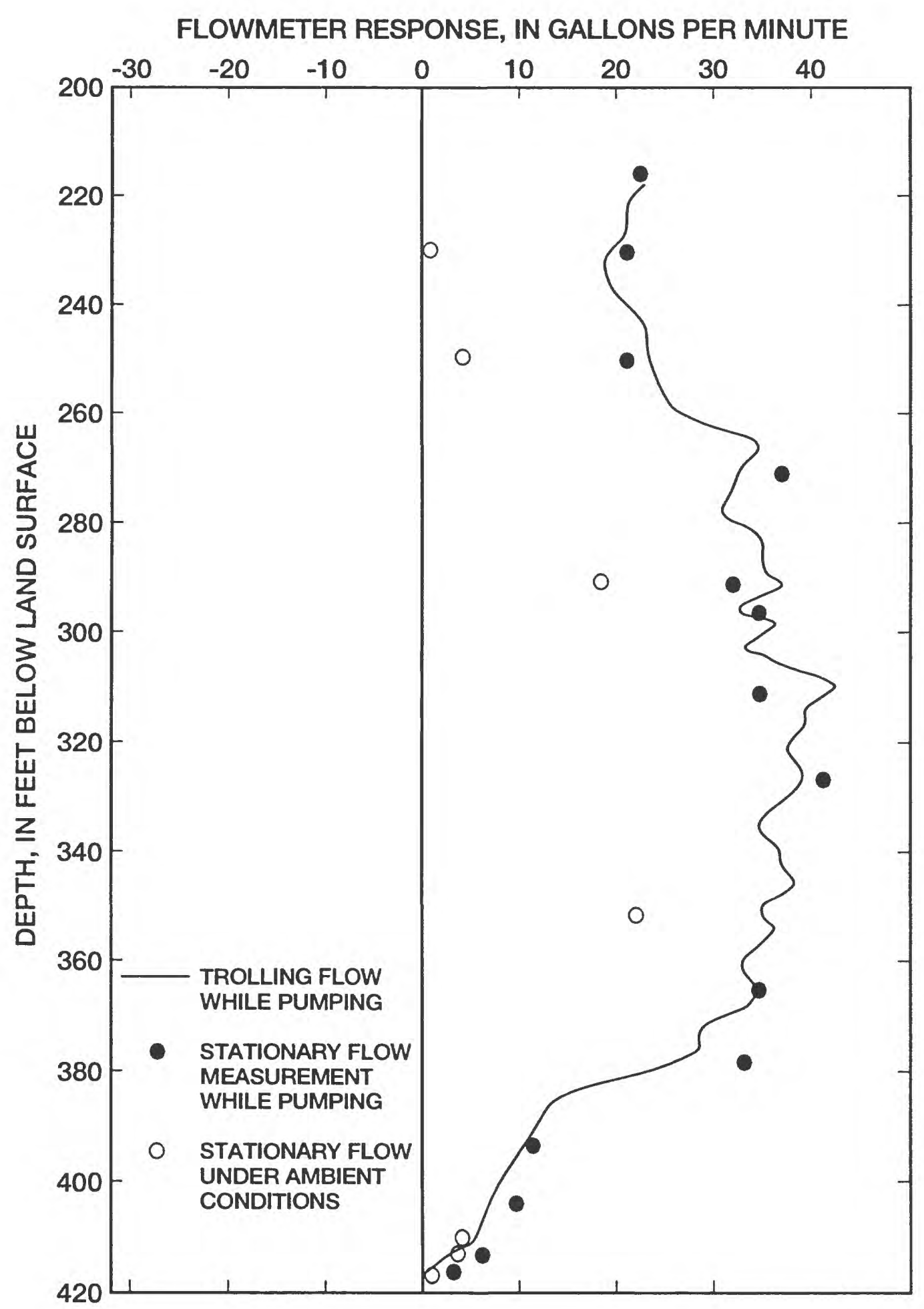

Figure 19. Heat-pulse flowmeter data obtained in borehole Obs F. 


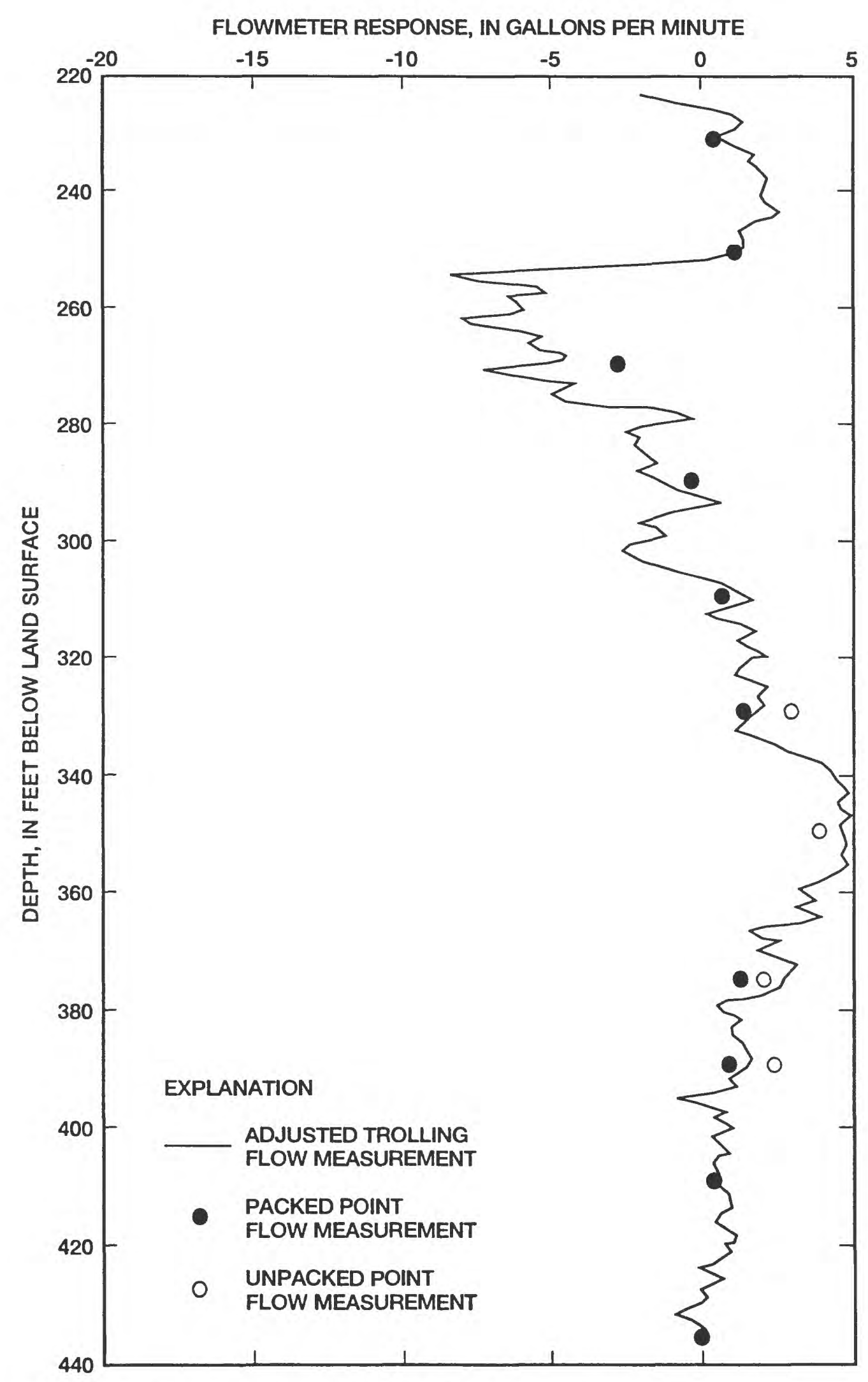

Figure 20. Heat-pulse flowmeter data obtained in borehole Obs $\mathrm{H}$. 


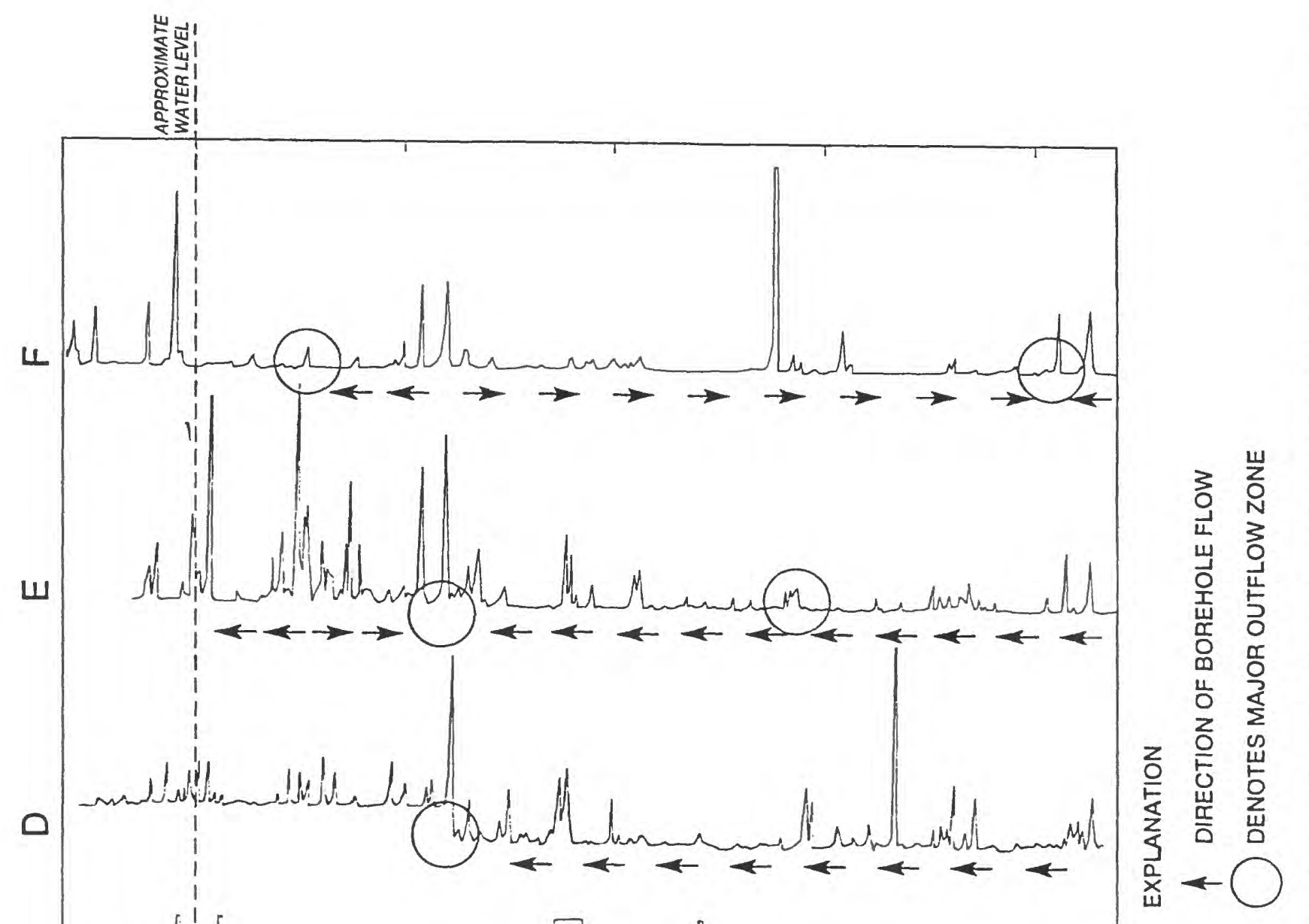

总

政

迆

ํㅗㅇ

足

오동

3

के

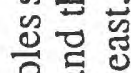

을 동

氙总

8 仓

E

흥

Z.

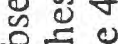

О 可

․ㅡㅇ

颌

สำ

तु

气

衣氖

क क :

응

ปั

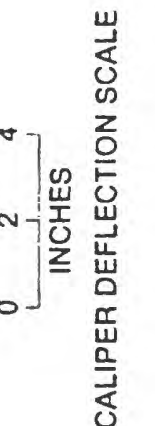

.

ปั

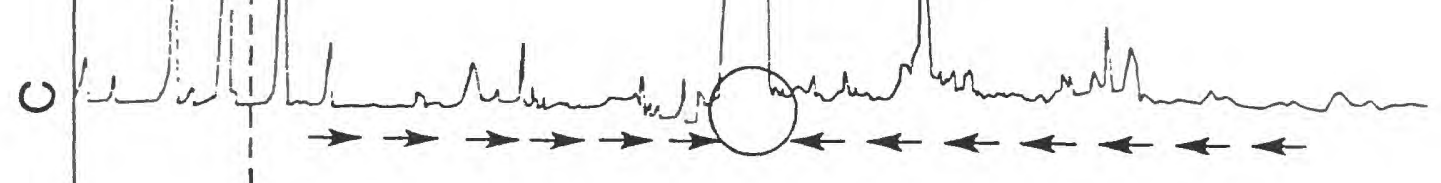

1111

(

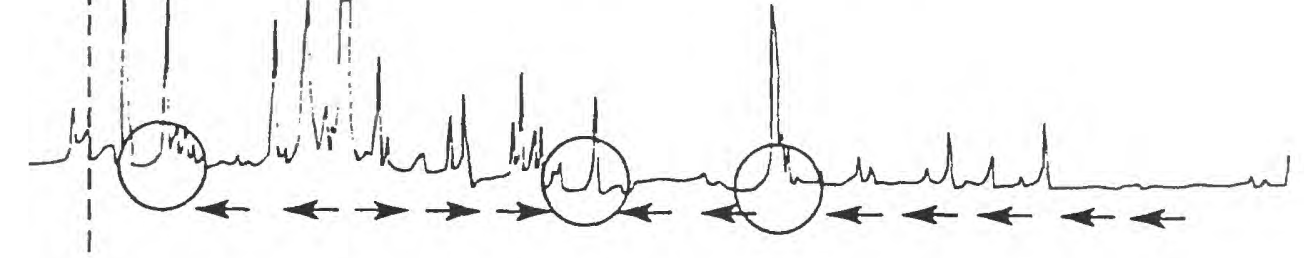

$\infty$

$<\underbrace{}_{-1}$

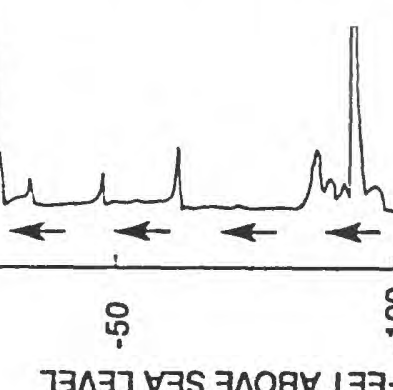

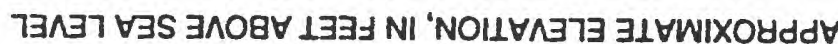

导芯

ธ岕

驱

తึ

包里

$\Xi 0$

品

ธี

定:

웡

르

芩

ए

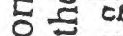

U ते

ते 


\section{Flow in the Waipahu Mahoe Boreholes During Borehole Pumping}

The distribution of inflow and outflow under ambient conditions in observation boreholes depends upon both the transmissivity of the producing zones and the hydraulic-head differences driving the measured flow. The ambiguity in the interpretation can be reduced if flows are measured during aquifer tests where the rate and location of pumping are known, and transmissivity can be uncoupled from the driving hydraulic-head gradients in the analysis of HPFM data.

Aquifer tests were conducted in three of the Waipahu Observation boreholes (Obs A, Obs B, and Obs E) by measuring flow in most of the Waipahu Observation boreholes while the borehole was being pumped by inserting a submersible pump above the logging tool. The results of the HPFM pumping tests are indicated in figures $14 \mathrm{C}$, 17, and 19. These results show that the borehole wall openings indicated on the caliper log that are associated with the largest inflow or outflow under ambient conditions are not always associated with the largest inflow during pumping.

\section{Logs from the Waipio Deep Observation Borehole}

Caliper and HPFM logs obtained in the Waipio Deep Observation borehole are given in figure 22. HPFM measurements indicated relatively small downflow over much of the upper part of the well bore. Not enough fixedmode data points could be obtained in the limited time available to constrain the details of this flow distribution, and the small magnitude of the flow resulted in a great deal of scatter in the trolling mode data. The data appear to indicate weak downflow in the intervals from 450 to 550 and from 850 to 1100 feet in depth, and upflow over the intervals from 550 to 850 and below 1100 feet in depth. This pattern of flow is consistent with the few fixed mode data points that show weak downflow above 1100 feet in depth, and weak upflow below 1100 feet in depth. Water sample data from this borehole indicate that the fluid column is fresh above about 1100 feet in depth, and saline below that depth. The presence of flow in the borehole may indicate that this depth is not a reliable indicator of the local depth to the freshwater/ salt-water interface in the aquifer. The measured depth of the contact in the well bore may have been influenced by the flow regime, and may differ from that of the freshwater/ salt-water transition before the borehole was drilled. 
conditions; flow data obtained on 7-26-88.

12. Televiewer image log interpretations and caliper logs for boreholes Obs A and Obs B

13. Digitally enhanced interval of televiewer $\log$ for borehole Obs B, indicating displays based on both amplitude and travel time of the reflected signal; caliper log shown for comparison

14. - 21. Graphs showing:

14. Heat-pulse flowmeter data obtained in borehole Obs A: (A) Flowmeter data obtained while trolling uphole with the packer deflated;

(B) Data after correction to account for bias of trolling speed;

(C) Interpretation of inflow and outflow based on interpretation of the flowmeter data;

(D) Stationary flowmeter data in table 2 plotted to show effects of changes over time and pumping on the velocity profile

15. Heat-pulse flowmeter data obtained in borehole Obs B

16. Heat-pulse flowmeter data obtained in borehole Obs $\mathrm{C}$

17. Heat-pulse flowmeter data obtained in borehole Obs D

18. Heat-pulse flowmeter data obtained in borehole Obs E.

19. Heat-pulse flowmeter data obtained in borehole Obs F.

20. Heat-pulse flowmeter data obtained in borehole $\mathrm{Obs} \mathrm{H}$

21. Correlation of openings indicated on the caliper logs for the Waipahu Observation boreholes showing the pattern of flow indicated by the interpretation of the flowmeter data; boreholes are all about 8 inches in diameter and the length scale for caliper deflections is given at the bottom of figure.

22. Caliper log and heat-pulse flowmeter measurements made while trolling in the Waipahu Deep

Observation borehole

23. Map showing location of borehole SOH-2 at the Pahoa, Hawaii study site

24. Conventional gamma, caliper, and BHTV logs for borehole SOH-2

25. Digitally enhanced interval of televiewer log from borehole SOH-2 showing individual fractures intersecting the well bore; caliper log shown for comparison.

26. Spinner flowmeter logs obtained in borehole SOH-2 showing distribution of downflow under ambient hydraulic-head conditions 


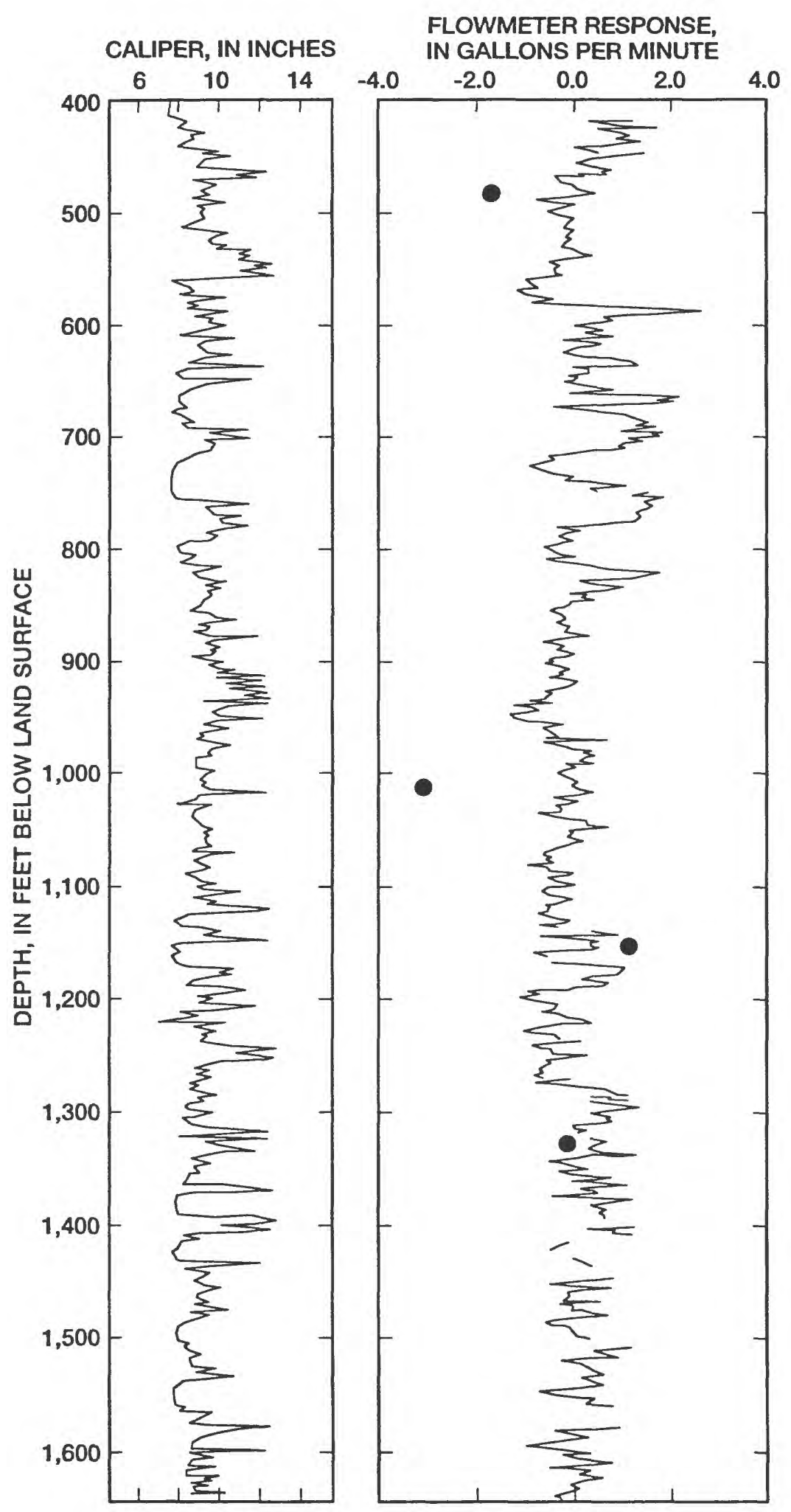

EXPLANATION

- stationary flow MEASUREMENTS

FLOW MEASUREMENTS MADE WHILE TROLLING

Figure 22. Caliper log compared to heat-pulse flowmeter measurements made while trolling in the Waipio Deep Observation borehole. 


\section{GEOPHYSICAL LOGGING NEAR PAHOA, HAWAII}

\section{Overview of Logging Activities at the Pahoa Site}

The University of Hawaii made the upper part of a deep scientific research borehole, Scientific Observation hole number 2 (SOH-2), available for logging by the U.S. Geological Survey (fig. 23). Although the purpose of the University of Hawaii study was the characterization of the deep geothermal reservoir in the Pahoa area, the purpose of the U.S.G.S. effort was the characterization of the hydrogeology of the shallow ground water circulation in the basalt flows penetrated by the first 2,000 feet (as originally projected) of drilling before installation of a surface casing. Several lost circulation zones delayed drilling during the first months of 1991 and required injection of cement into the formation before drilling could be resumed. Drilling was eventually stopped at a depth of about 1,900 feet where the driller felt that an effective seal could be established after installation of the surface casing. Borehole SOH-2 was logged in February 1991 during a period when the 8-in-diameter borehole was left open before installation of casing. Caliper, gamma, BHTV and spinner flowmeter logs were run in the borehole. The HPFM was not run because temperatures were expected to exceed those over which the HPFM functions, and because a downhole packer was not available to allow use of the HPFM in a 8-inch-diameter borehole. The BHTV was only run from 1,000 to 1,870 feet in depth, because the image logs showed that cementation of borehole wall openings precluded obtaining useful logs above these depths. All BHTV logs were obtained by operating with a U.S. Geological Survey logging cable and winch brought to the site for this project, eliminating the equipmentinduced noise experienced in the Waipahu logging. 
ह हा
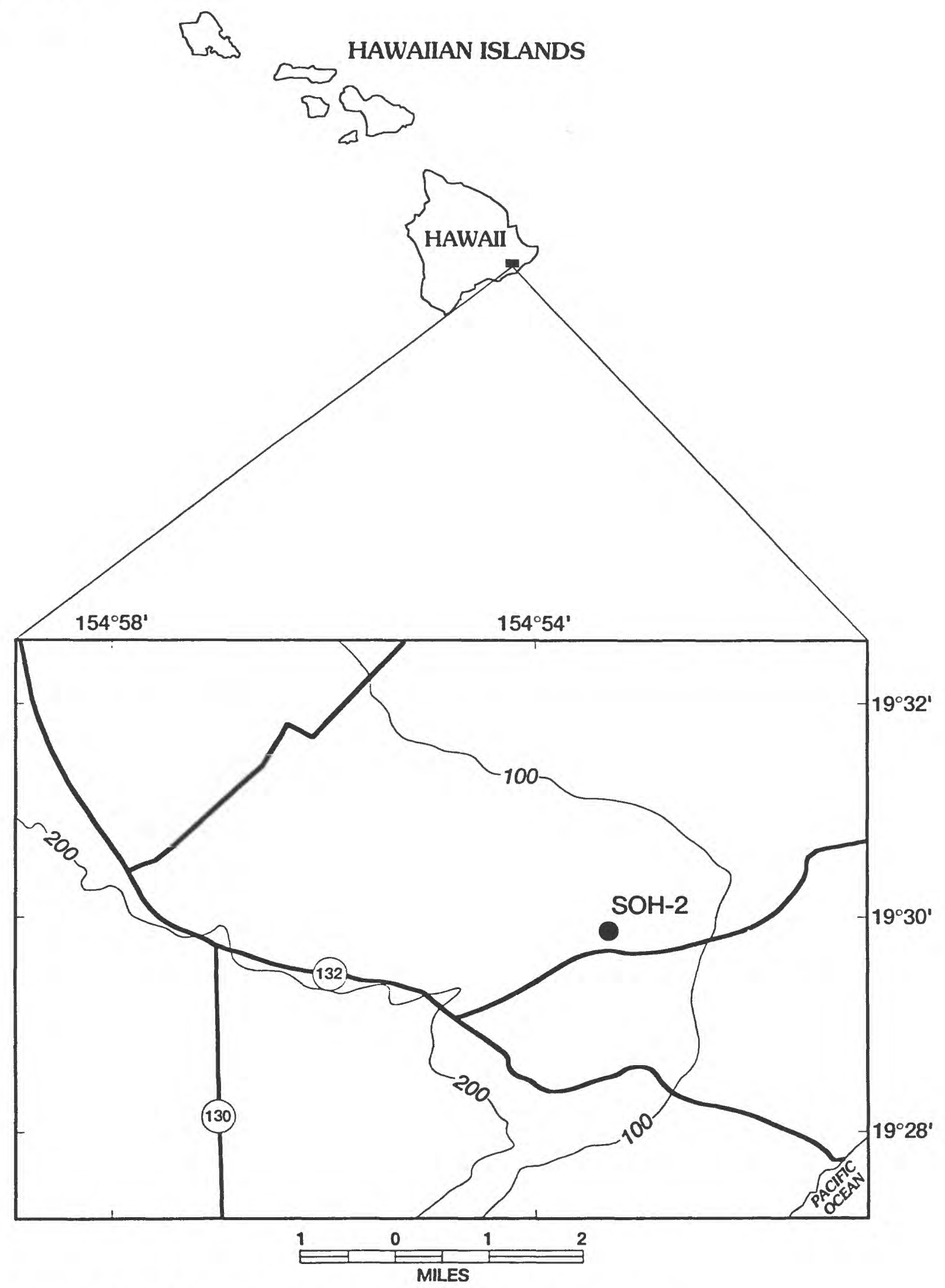

Figure 23. Location of borehole SOH-2 at the Pahoa, Hawaii study site. 


\section{Conventional Geophysical Logs in Borehole SOH-2}

Caliper and natural gamma logs were run in borehole SOH-2 (fig. 24). The caliper log showed a number of large openings in the borehole wall below 1,200 feet in depth, and many of these openings were assumed to correspond to clinker zones between flows similar to those indicated by the logs from the Waipahu boreholes. The caliper log indicated almost no openings were present above 1,200 feet in depth, probably because all of the openings originally intersected by the borehole had been filled with cement during efforts to control lost circulation. 

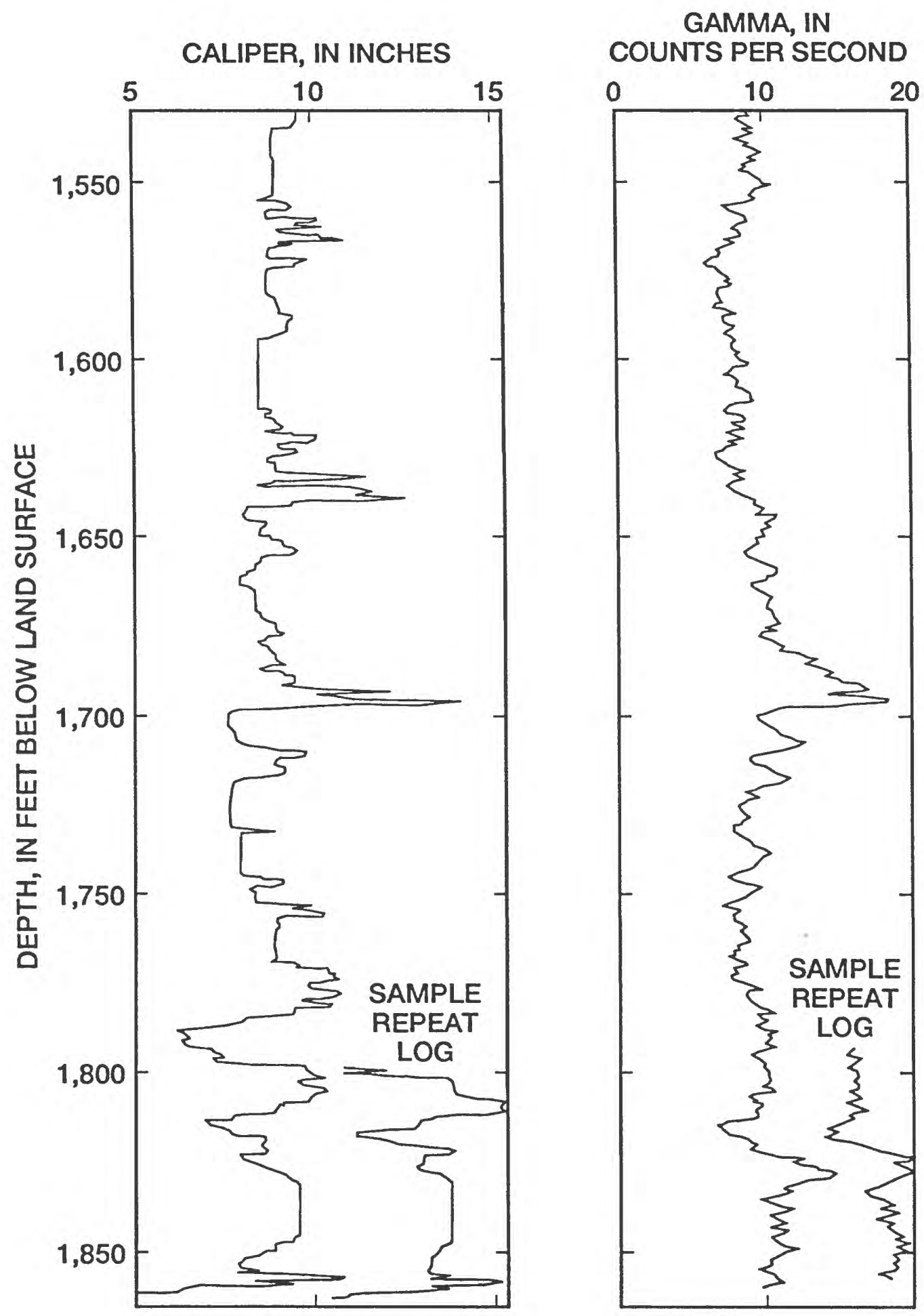

TELEVIEWER

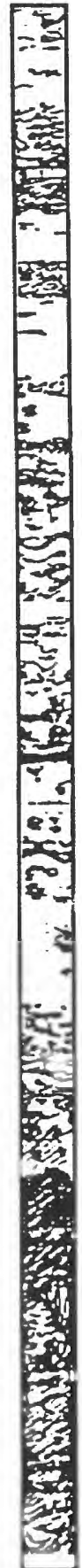

Figure 24. Conventional gamma, caliper, and BHTV logs for borehole SOH-2. 
Oceanic basalts similar to those known to make up most of the subsurface in Hawaii are known to contain relatively little natural gamma activity (Keys, 1990). Therefore, there was concern that gamma logs would not differentiate between different geological formations in borehole SOH-2. One possible cause of poor gamma logs in such formations is attributed to the speed with which the logging probe is moved during logging. Gamma probes are designed to average nuclear counts over a specific counting period denoted as the time constant (Keys, 1990). The time constant needs to be made large enough so that averaged gamma counts represent a reliable average of the rate at which gamma photons are being emitted by the formation. If the time constant is made too large, the logging probe will move through several sample volumes during the counting period. In situations where unusually low gamma activity is expected, logging speed needs to be reduced so that a large time constant can be used without degrading the vertical resolution of the log.

After some experimentation in borehole SOH-2, useful gamma logs were made with a time constant of 5 seconds and a logging speed of 10 feet per minute, corresponding with $0.8 \mathrm{ft}$ of vertical travel during the time constant. These values represent the upper limit of logging speed and the lower limit of time constant for the formations in the study area. This is demonstrated by considering the average gamma activity of 10 counts per second given in figure 24. The error (standard deviation of repeat measurements) associated with nuclear counting is given by the formula:

$$
\sigma=\frac{\sqrt{N}}{N}
$$

where $\sigma$ is the standard error, and $\mathrm{N}$ is the number of gamma photons measured in a counting period. If a 1 -sec time constant were used, this error would be more than 30 percent, which is unacceptable. Using a time constant of 5 seconds, an average of 50 gammas would be counted in each measurement period, giving a standard error of about 14 percent. This error was considered barely acceptable. At the same time, the gamma logging probe is known to measure the gamma activity of a sample volume extending about 1 foot along the borehole (Keys, 1990). Assuming this ideal vertical resolution, the logging probe cannot be moved over a distance of more than 1 foot in one time constant without degrading bed resolution. Therefore, logging speed needed to be no more than about 10 feet per minute when logging borehole SOH-2 to insure that the logging probe did not move more than 1 foot in 5 seconds.

The effectiveness of using a 5-sec time constant and a logging speed of $10 \mathrm{ft} / \mathrm{min}$ in logging borehole SOH-2 is indicated by the repeatability of the gamma log in figure 24 . The natural gamma log showed that beds of relatively higher gamma activity could be identified in the borehole, and the character of the log was reproduced by a short repeat run under the same conditions. The gamma log also shows that most basalt flows encountered by borehole SOH-2 had a natural gamma activity of about 10 counts per second, with local highs of about 20 counts per second. Comparing the gamma log with the caliper log indicates that these local highs are associated with openings between basalt layers, and may represent the presence of clay minerals in incipient soil horizons.

\section{Acoustic Borehole Televiewer Logs in Borehole SOH-2}

The BHTV log was run in borehole SOH-2, and the unprocessed signal from the logging probe was recorded on a modified video-cassette recorder. This analog signal was subsequently digitized and processed and displayed as an amplitude image (fig. 25). The BHTV log data are shown with the caliper and gamma logs in figure 24. The BHTV log shows that some of the openings indicated on the caliper log are associated with interbed zones, whereas others are associated with fractures and the contacts between intrusions and surrounding rock. A summary of the strike and dip of such features cutting across horizontal flows is given in table 4. The character of the BHTV log data indicate the great difference in geological environment between the basalts at Waipahu and the basalts at Pahoa. The Waipahu basalts were apparently ponded in the relatively flat and structurally undisturbed area between two major volcanic eruption centers (Hunt and others, 1988). The Pahoa basalts are located in the area of active eruptions and rifting along the eastern extension of the Kilauea Rift zone, and have been subjected to faulting and intrusion. 

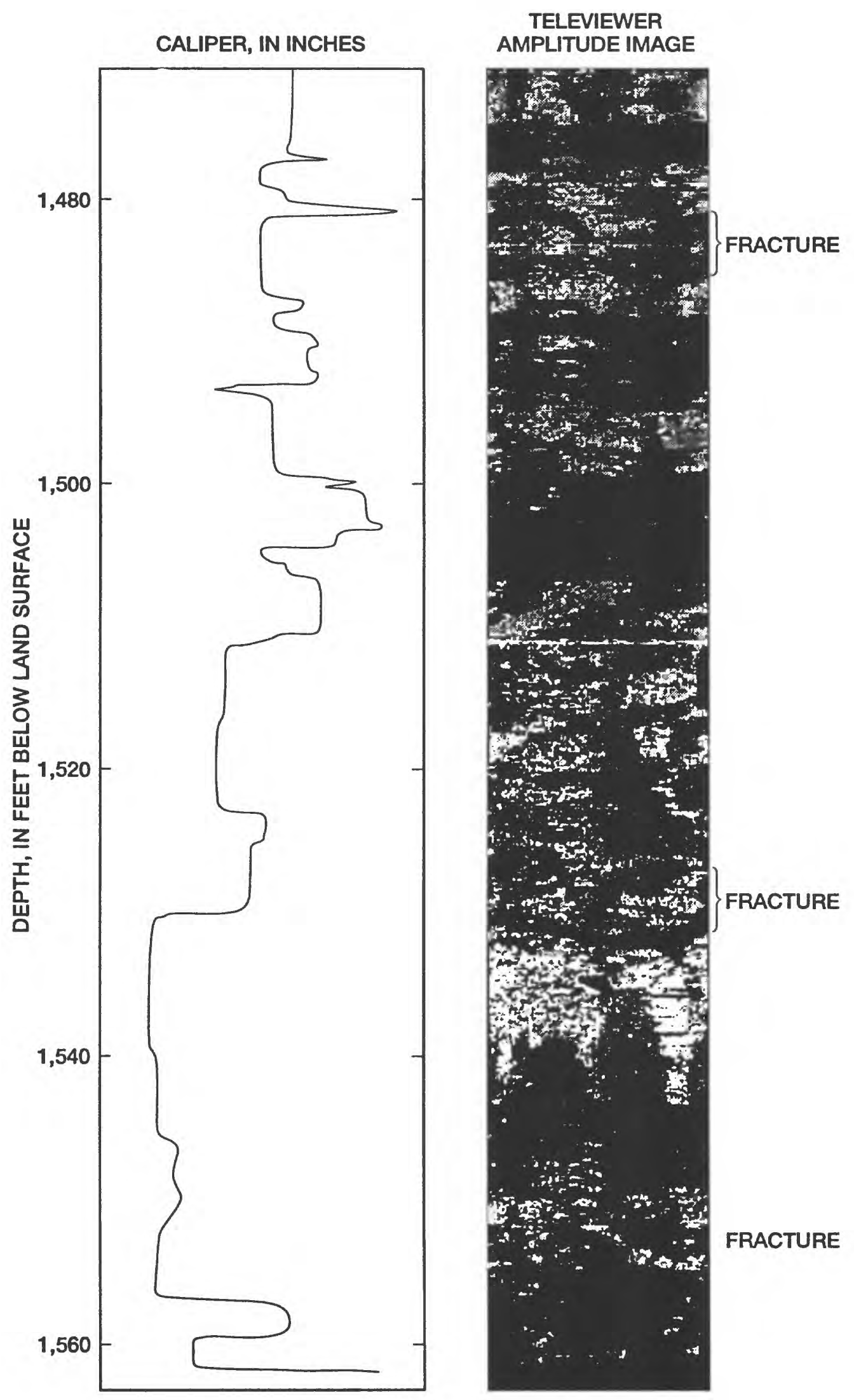

Figure 25. Digitally enhanced interval of televiewer log from borehole SOH-2 showing individual fractures intersecting the well bore; caliper log shown for comparison. 
Table 4. Orientations of features identified on televiewer logs obtained in borehole SOH-2 at the Pahoa, Hawaii site

\begin{tabular}{|c|c|c|c|}
\hline Depth' ${ }^{1}$ & $\begin{array}{c}\text { Strike }^{2} \\
\text { (degrees) }\end{array}$ & $\begin{array}{c}\text { Dip }^{3} \\
\text { (degrees) }\end{array}$ & Feature interpretation 4 \\
\hline 1102 & 265 & 79 & Fracture \\
\hline 1129 & 25 & 76 & Fracture \\
\hline 1131 & 117 & 76 & Fracture \\
\hline 1233 & 133 & 76 & Fracture \\
\hline 1267 & 221 & 66 & Fracture \\
\hline 1276 & 194 & 66 & Fracture \\
\hline 1295 & 194 & 66 & Intrusion \\
\hline 1315 & 190 & 66 & Intrusion \\
\hline 1329 & 357 & 82 & Fracture \\
\hline 1345 & 275 & 75 & Fracture \\
\hline 1362 & 150 & 66 & Fracture \\
\hline 1377 & 145 & 61 & Fracture \\
\hline 1435 & 145 & 61 & Fracture \\
\hline 1481 & 165 & 77 & Fracture \\
\hline 1487 & 187 & 61 & Fracture \\
\hline 1528 & 147 & 72 & Fracture \\
\hline 1551 & 215 & 72 & Fracture \\
\hline 1641 & 85 & 75 & Fracture \\
\hline 1663 & 232 & 66 & Fracture \\
\hline 1712 & 214 & 75 & Fracture \\
\hline
\end{tabular}

${ }^{1}$ Depth of projected intersection of fracture or intrusion plane with centerline of borehole; depths referenced with respect to land surface, $\pm 1.0 \mathrm{ft}$.

${ }^{2}$ Strike is given according to right hand rule, where downdip azimuth is given by strike plus 90 degrees; values given to \pm 20 degrees.

${ }^{3}$ Dip azimuth determined by adding 90 degrees to strike; values given to \pm 20 degrees.

${ }^{4}$ Feature type interpreted from appearance on televiewer log image. 


\section{Spinner Flowmeter Logs in Borehole SOH-2}

Although the HPFM could not be used in borehole SOH-2, a conventional spinner flowmeter was run to check for the presence of flows large enough to be measured with this device (fig. 26). The spinner flowmeter requires flows greater than several feet per minute to turn the impeller on a stationary probe; slightly more sensitive measurements can be obtained by comparing the logs when the probe is moved up and down the borehole. The spinner flowmeter did not indicate flow in borehole SOH-2 when the probe was stationary. However, a comparison of logs made while trolling the spinner up and down the borehole did indicate a significant difference in flow. There is usually a difference in the impeller rotation rate between upward and downward probe motion in a stagnant fluid column because the body of the probe shields the impeller from the effects of probe movement during upward logging. In the interval above 800 feet in depth, there was the expected difference in impeller rotation rate between upward and downward trolling (upward trolling response about $20 \%$ less than the downward trolling response). However, the impeller rotation rates measured while trolling up appeared greater than those measured trolling down over the interval from about 800 to 1,780 feet in depth, indicating a downflow over that interval. Using previously established calibration values for this flowmeter, a downflow of from 5 to 10 gallons per minute was estimated. The depth where the downflow appears to exit the borehole (fig. 26) corresponds with an abrupt increase in the recorded intensity of the BHTV signal (fig. 24). This was originally interpreted as a change in borehole wall texture. The spinner flowmeter data indicate that freshwater from a shallow inflow zone had filled the well bore down to a depth of about 1,780 feet. The well bore was apparently filled with viscous, acoustically attenuating drilling mud below 1,780 feet in depth. The presence of this mud and not a change in borehole wall properties apparently accounts for the appearance of the BHTV log at the time of logging. 


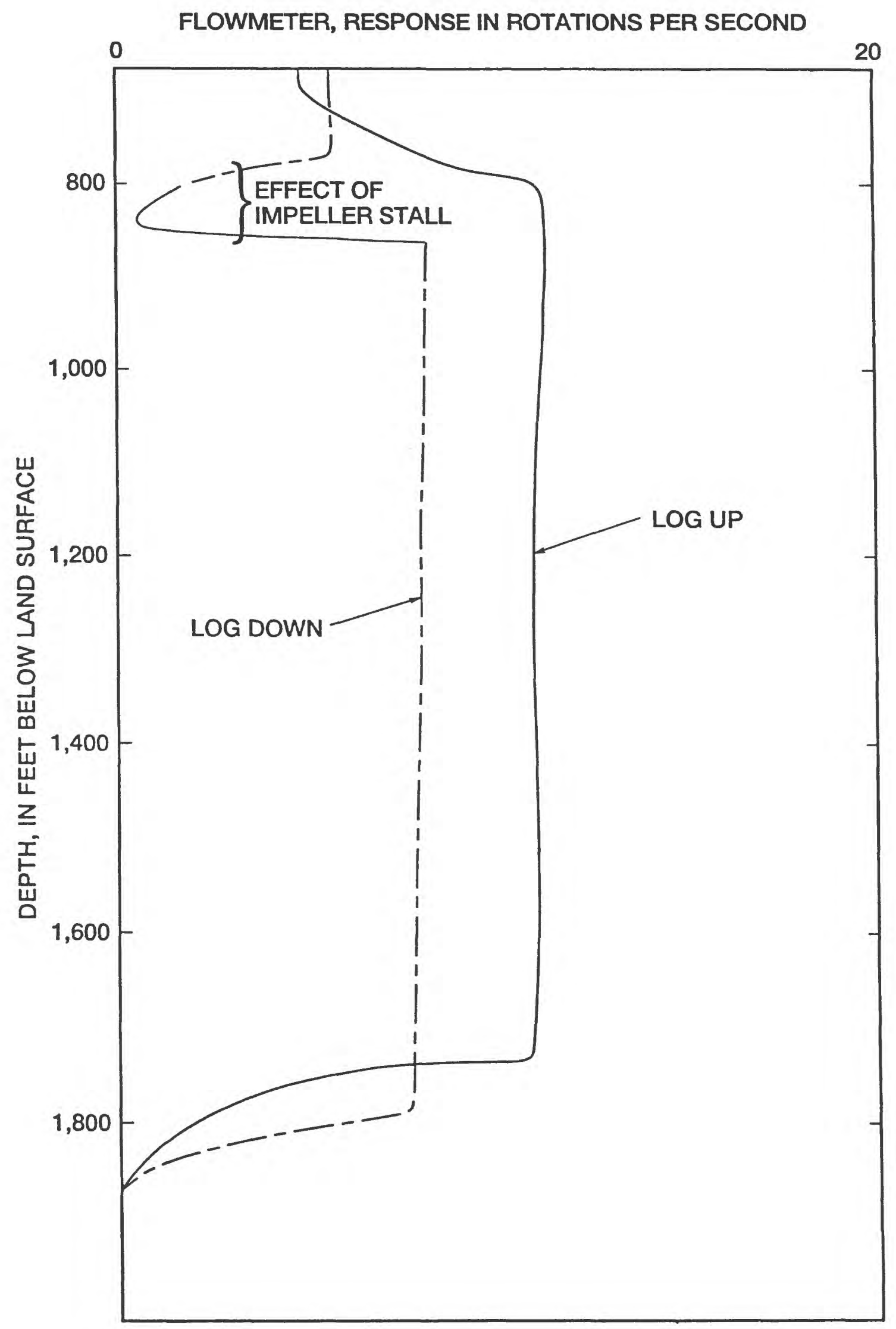

Figure 26. Spinner flowmeter logs obtained in borehole SOH-2 showing distribution of downflow under ambient hydraulic-head conditions. 


\section{Tables}

1. Locations and separations of Mahoe Pumping Station production wells and Waipahu Mahoe Observation boreholes

2. Summary of heat-pulse flowmeter logs at the Waipahu wellfield ................................................................ 25

3. Heat-pulse flowmeter data from the Waipahu boreholes ................................................................................ 27

4. Orientations of features identified on televiewer logs obtained in borehole SOH-2 at the Pahoa, 
The upward trolling spinner flow data indicate that water enters the well bore at about 800 feet in depth. Inflow is not indicated by a similar change in the downward trolling data at that depth. Instead, the downward data show a sharp drop in impeller rotation, after which the rotation "catches up" to a reduced rate. This drop is attributed to the logging tool being temporarily caught on a "ledge" during the downward logging run. This ledge may represent the bottom of the fracture or flow zone associated with the inflow. Both upward and downward trolling measurements apparently indicate flow in borehole SOH-2 under the naturally occurring hydraulic-head conditions. This downflow would be expected in an area characterized by abundant rainfall and downward decreasing hydraulic heads produced by recharge. Constraints on logging time precluded additional BHTV logging after the flow logging had indicated inflow in the upper part of borehole SOH-2 where BHTV logs had not been obtained.

\section{SUMMARY}

Two relatively new geophysical logging techniques, the digitally enhanced borehole acoustic televiewer and the heat-pulse flowmeter, were tested from 1986 to 1991 at two sites in Hawaii. Although these data were generated in an effort to test and improve these two logging techniques, the measurements are of interest to hydrologists studying the aquifers in Hawaii. This report presents a review of the measurements conducted during this effort and summarizes the data obtained in a form designed to make that data available to hydrogeologists studying the movement of ground water in Hawaiian aquifers.

The flowmeter data obtained at the Waipahu site indicate the distribution of openings between relatively dense and impermeable basalt flows. The data indicate the pattern of flow induced along seven observation boreholes that provide conduits between interbed zones in the vicinity of a water-supply pumping station. The televiewer image logs obtained in some of these boreholes do not show any significant vertical or steeply dipping fractures that might allow communication across the dense interior of basalt flows. It is concluded that horizontal flow in the aquifer is facilitated by the lateral continuity of interbed zones, but that vertical flow occurs by a much more tortuous route around the edges of flows.

Acoustic televiewer logs obtained in the upper part of a deep scientific observation borehole at the Pahoa site showed that a number of steeply dipping fractures and dikes cut across the basalt flows deposited in this area. The difference in televiewer logs obtained at Pahoa and Waipahu is probably explained by the difference in depositional environments, because basalts at Waipahu were deposited in a relatively undisturbed area between two eruptive centers, whereas the basalts at Pahoa were deposited on an active part of the Kilauea Rift Zone. Comparison of the televiewer log with the caliper log for borehole SOH-2 show that the televiewer is useful in distinguishing between openings on the caliper log associated with clinker zones and those associated with fractures and intrusive contacts. Comparison of the televiewer log with the gamma log indicates that small maxima in gamma activity may be associated with incipient soil horizons or weathered clinker zones.

Although flow under ambient hydraulic-head conditions in the Waipahu Mahoe boreholes was attributed to hydraulic gradients associated with water production from the nearby Mahoe Pumping Station, flow in the Waipio Deep Observation borehole on Oahu and the Scientific Observation borehole on Hawaii are attributed to the effects of natural recharge and downward decreasing hydraulic heads associated with that recharge.

\section{REFERENCES}

Barton, C.A., Tesler, L.G., and Zoback, M.D., 1991, Interactive image analysis of borehole televiewer data, in Sengupta, I.P.A.S., ed., Automated Pattern Analysis in Petroleum Exploration: New York, Springer-Verlag, p. 265-284.

Hearst, J.R., and Nelson, P.H., 1985, Well logging for physical properties: New York, McGraw-Hill, 571 p.

Hess, A.E., 1986, Identifying hydraulically conductive fractures with a slow-velocity borehole flowmeter: Canadian Geotechnical Journal, v. 23, p. 69-78.

Hess, A.E., and Paillet, F.L., 1990a, Characterizing flow paths and permeability distribution in fractured-rock aquifers using a sensitive, thermal borehole flowmeter, in Molz, F.J., and others, eds., Proceedings of the conference on new field techniques for quantifying the physical and chemical properties of heterogeneous aquifers: Dublin, $\mathrm{OH}$, National Water Well Association, 1989, p. 445-461. 
Hess, A.E., and Paillet, F.L., 1990b, Applications of the thermal-pulse flowmeter in the hydraulic characterization of fractured rocks, in Geophysical Applications for Geotechnical Investigations: American Society for Testing and Materials, Standard Technical Publication 1101, p. 99-112.

Hunt, C.D., Ewart, C.J., and Voss, C.I., 1988, Region 27, Hawaiian Island: Geological Society of America, v. O-2, Hydrogeology, p. 255-262.

Keys, W.S., 1979, Borehole geophysics in igneous and metamorphic rocks, in Society of Professional Well Log Analysts Annual Logging Symposium, 20th, Tulsa, Okla., Transactions, 1979, Paper OO.

Keys, W.S., 1990, Borehole geophysics applied to ground-water investigations: Techniques of Water-Resources Investigations of the U.S. Geological Survey, bk. 2, chap. E2, 150 pp.

Kierstein, R.A., 1983, True location and orientation of fractures logging with the acoustic televiewer (including programs to correct fracture orientation): U.S. Geological Survey Water-Resources Investigations Report 83-4275, 73 p.

Paillet, F.L., 1991, Use of geophysical well-logs in evaluating crystalline rocks for siting of radioactive waste repositories: The Log Analyst, v. 33, no. 2, p. 85-107.

Paillet, F.L., and others, eds., 1990, Borehole imaging-SPWLA Reprint Volume: Houston, Tex., Society of Professional Well Log Analysts, $472 \mathrm{p}$.

Paillet, F.L., Keys, W.S., and Hess, A.E., 1985, Effects of lithology on televiewer-log quality and fracture interpretation: Society of Professional Well Log Analyst Annual Logging Symposium, 26th, Dallas, Tex., 1985, Transactions, p. JJJ1-JJJ30.

Visher, F.N., and Mink, J.F., 1964, Ground-water resources in southern Oahu, Hawaii: U.S. Geological Survey Water Supply Paper 1778, $133 \mathrm{p}$.

Zemanek, Joseph, Glenn, E.E., Norton, L.J., and Caldwell, R.L., 1970, Formation evaluation by inspection with the borehole televiewer: Geophysics, v. 35, p. 254-269. 





\begin{tabular}{rll}
\hline Multiply & By & To obtain \\
\hline foot (ft) & & meter \\
inch (in.) & 0.3048 & millimeter \\
gallon & 25.4 & liter \\
gallon per minute (gal/min) & 3.785 & liter per second \\
mile (mi) & 0.06309 & kilometer \\
\hline
\end{tabular}

The following terms and abbreviations also are used in this report:

ohm-meters (ohm-m)

megahertz $(\mathrm{MHz})$

Degree Celsius $\left({ }^{\circ} \mathrm{C}\right)$ may be converted to degree Fahrenheit $\left({ }^{\circ} \mathrm{F}\right)$ by using the following equation:

$$
{ }^{\circ} \mathrm{F}=9 / 5\left({ }^{\circ} \mathrm{C}\right)+32 \text {. }
$$

Degree Fahrenheit $\left({ }^{\circ} \mathrm{F}\right)$ may be converted to degree Celsius $\left({ }^{\circ} \mathrm{C}\right)$ by using the following equation:

$$
{ }^{\circ} \mathrm{C}=5 / 9\left({ }^{\circ} \mathrm{F}-32\right) \text {. }
$$

Sea level: In this report "sea level" refers to the National Geodetic Vertical Datum of 1929 (NGVD of 1929)--a geodetic datum derived from a general adjustment of the first-order level nets of both the United States and Canada, formerly called Sea Level Datum of 1929. 
